\title{
GUIDELINES
}

\section{Surviving sepsis campaign international guidelines for the management of septic shock and sepsis-associated organ dysfunction in children}

Scott L. Weiss ${ }^{*}$, Mark J. Peters ${ }^{2}$, Waleed Alhazzani ${ }^{3,4}$, Michael S. D. Agus ${ }^{5}$, Heidi R. Flori ${ }^{6}$, David P. Inwald ${ }^{7}$, Simon Nadel ${ }^{7}$, Luregn J. Schlapbach ${ }^{8}$, Robert C. Tasker ${ }^{5}$, Andrew C. Argent ${ }^{9}$, Joe Brierley ${ }^{2}$, Joseph Carcillo ${ }^{10}$, Enitan D. Carrol ${ }^{11}$, Christopher L. Carroll12, Ira M. Cheifetz ${ }^{13}$, Karen Choong ${ }^{3,4}$, Jeffry J. Cies ${ }^{14}$, Andrea T. Cruz ${ }^{15}$, Daniele De Luca ${ }^{16,17}$, Akash Deep ${ }^{18}$, Saul N. Faust ${ }^{19}$, Claudio Flauzino De Oliveira ${ }^{20}$, Mark W. Hall ${ }^{21}$, Paul Ishimine ${ }^{22}$, Etienne Javouhey ${ }^{23}$, Koen F. M. Joosten ${ }^{24}$, Poonam Joshi ${ }^{25}$, Oliver Karam ${ }^{26}$, Martin C. J. Kneyber ${ }^{27}$, Joris Lemson ${ }^{28}$, Graeme MacLaren ${ }^{29,30}$, Nilesh M. Mehta ${ }^{31}$, Morten Hylander Møller ${ }^{32}$, Christopher J. L. Newth ${ }^{33}$, Trung C. Nguyen ${ }^{15}$, Akira Nishisaki ${ }^{1}$, Mark E. Nunnally ${ }^{34}$, Margaret M. Parker ${ }^{35}$, Raina M. Paul ${ }^{36}$, Adrienne G. Randolph ${ }^{31}$, Suchitra Ranjit ${ }^{37}$, Lewis H. Romer ${ }^{38}$, Halden F. Scott ${ }^{39}$, Lyvonne N. Tume ${ }^{40}$, Judy T. Verger ${ }^{1,41}$, Eric A. Williams ${ }^{15}$, Joshua Wolf ${ }^{42}$, Hector R. Wong ${ }^{43}$, Jerry J. Zimmerman ${ }^{44}$, Niranjan Kissoon ${ }^{45}$ and Pierre Tissieres ${ }^{16,46}$

\section{Abstract}

Objectives: To develop evidence-based recommendations for clinicians caring for children (including infants, school-aged children, and adolescents) with septic shock and other sepsis-associated organ dysfunction.

Design: A panel of 49 international experts, representing 12 international organizations, as well as three methodologists and three public members was convened. Panel members assembled at key international meetings (for those

\footnotetext{
*Correspondence: WeissS@email.chop.edu

${ }^{1}$ Children's Hospital of Philadelphia, University of Pennsylvania Perelman School of Medicine, Philadelphia, PA, USA

Full author information is available at the end of the article

These guidelines are simultaneously being published in Pediatric Critical Care Medicine (DOI: https://doi.org/10.1097/PCC.0000000000002198) and Intensive Care Medicine (DOI: https://doi.org/10.1007/s00134-019-05878-6)

The following sponsoring organizations with formal liaison appointees endorse this guideline: American Academy of Pediatrics; American Association of Critical-Care Nurses; American College of Chest Physicians; American College of Emergency Physicians; American Thoracic Society; Australian and New Zealand Intensive Care Society; Canadian Critical Care Society; European Society of Neonatal and Pediatric Intensive
}

Care; Pediatric Infectious Diseases Society; Scandinavian Society of Anaesthesiology and Intensive Care Medicine; Society of Infectious Diseases Pharmacists; UK Sepsis Trust; World Federation of Pediatric Intensive and Critical Care Societies.

These guidelines were solely funded by the European Society of Intensive Care Medicine and the Society of Critical Care Medicine.

The Society of Critical Care Medicine guidelines are intended for general information only, are not medical advice, and do not replace professional advice, which should be sought for any medical condition. The full disclaimer for guidelines can be accessed at https://www.sccm.org/Resea rch/Guidelines/Guidelines. 
panel members attending the conference), and a stand-alone meeting was held for all panel members in November 2018. A formal conflict-of-interest policy was developed at the onset of the process and enforced throughout. Teleconferences and electronic-based discussion among the chairs, co-chairs, methodologists, and group heads, as well as within subgroups, served as an integral part of the guideline development process.

Methods: The panel consisted of six subgroups: recognition and management of infection, hemodynamics and resuscitation, ventilation, endocrine and metabolic therapies, adjunctive therapies, and research priorities. We conducted a systematic review for each Population, Intervention, Control, and Outcomes question to identify the best available evidence, statistically summarized the evidence, and then assessed the quality of evidence using the Grading of Recommendations Assessment, Development, and Evaluation approach. We used the evidence-to-decision framework to formulate recommendations as strong or weak, or as a best practice statement. In addition, "in our practice" statements were included when evidence was inconclusive to issue a recommendation, but the panel felt that some guidance based on practice patterns may be appropriate.

Results: The panel provided 77 statements on the management and resuscitation of children with septic shock and other sepsis-associated organ dysfunction. Overall, six were strong recommendations, 49 were weak recommendations, and nine were best-practice statements. For 13 questions, no recommendations could be made; but, for 10 of these, "in our practice" statements were provided. In addition, 52 research priorities were identified.

Conclusions: A large cohort of international experts was able to achieve consensus regarding many recommendations for the best care of children with sepsis, acknowledging that most aspects of care had relatively low quality of evidence resulting in the frequent issuance of weak recommendations. Despite this challenge, these recommendations regarding the management of children with septic shock and other sepsis-associated organ dysfunction provide a foundation for consistent care to improve outcomes and inform future research.

Keywords: Evidence-based medicine, Grading of Recommendations Assessment, Development and Evaluation criteria, Guidelines, Infection, Pediatrics, Sepsis, Septic shock, Surviving Sepsis Campaign

\section{Introduction}

Sepsis is a leading cause of morbidity, mortality, and healthcare utilization for children worldwide. Globally, an estimated 22 cases of childhood sepsis per 100,000 person-years and 2202 cases of neonatal sepsis per 100,000 live births occur, translating into 1.2 million cases of childhood sepsis per year [1]. More than $4 \%$ of all hospitalized patients less than 18 years and $\sim 8$ of patients admitted to PICUs in high-income countries have sepsis [2-6]. Mortality for children with sepsis ranges from $4 \%$ to as high as $50 \%$, depending on illness severity, risk factors, and geographic location [2, 3, 7-9]. The majority of children who die of sepsis suffer from refractory shock and/or multiple organ dysfunction syndrome, with many deaths occurring within the initial 48-72 $\mathrm{h}$ of treatment [10-13]. Early identification and appropriate resuscitation and management are therefore critical to optimizing outcomes for children with sepsis.

In 2001, the Surviving Sepsis Campaign (SSC) was formed by the Society of Critical Care Medicine (SCCM), European Society of Intensive Care Medicine (ESICM), and the International Sepsis Forum. A primary aim of the SSC was to develop evidenced-based guidelines and recommendations for the resuscitation and management of patients with sepsis. The initial guidelines were published in 2004 and have been reviewed and updated every four years thereafter. Following the 2016 edition, SCCM and ESICM reaffirmed their commitment to evidence-based guidelines for all patients by forming separate task forces dedicated to guidelines for adults and children.

The objective of the SCCM/ESICM "Surviving Sepsis Campaign International Guidelines for the Management of Septic Shock and Sepsis-associated Organ Dysfunction in Children" is to provide guidance for clinicians caring for children (including infants, school-aged children, and adolescents) with septic shock and other sepsis-associated organ dysfunction. We sought to leverage the expertise of a clinical and methodology team to create comprehensive evidence-based recommendations for the recognition and management of children with septic shock or other sepsis-associated acute organ dysfunction. Recommendations from these guidelines are based on the best current evidence but cannot replace the clinician's decision-making capability when presented with a patient's unique set of clinical variables. Recommendations are intended to guide "best practice" rather than to establish a treatment algorithm or to define standard of care. These guidelines are appropriate for treating septic shock and other sepsis-associated organ dysfunction in a hospital, emergency, or acute care setting, although some may be applicable elsewhere. Although recommendations were developed without consideration to 
availability of resources, we acknowledge that variation within and across healthcare systems and geographic regions will determine the practical application of these guidelines.

Although several recommendations for the care of children with sepsis and septic shock have been previously published [14-16], these new guidelines are not intended to update or iterate on these prior documents. Instead, it was the aim of SCCM/ESICM "Surviving Sepsis Campaign" to provide an evidence-based approach to the management of septic shock and other sepsis-associated organ dysfunction in children using a comprehensive and transparent methodologic approach by a panel with geographic and professional diversity.

\section{Methodology}

\section{Definitions}

In 2005, the International Pediatric Sepsis Consensus Conference published definitions and criteria for sepsis, severe sepsis, and septic shock in children based on prevailing views of adult sepsis at the time with modifications for physiology based on age and maturational considerations [17]. In 2016, new adult definitions and criteria were published (Sepsis-3) with "sepsis" defined as life-threatening organ dysfunction caused by a dysregulated host response to infection and "septic shock" the subset of sepsis with circulatory and cellular/metabolic dysfunction associated with a higher risk of mortality [18]. The term "severe sepsis" was replaced by this new definition of sepsis. Although application of Sepsis-3 to children has been attempted [19, 20], formal revisions to the 2005 pediatric sepsis definitions remain pending [21]. Therefore, the majority of studies used to establish evidence for these guidelines referred to the 2005 nomenclature in which severe sepsis was defined as (1) greater than or equal to 2 age-based systemic inflammatory response syndrome (SIRS) criteria, (2) confirmed or suspected invasive infection, and (3) cardiovascular dysfunction, acute respiratory distress syndrome (ARDS), or greater than or equal to 2 noncardiovascular organ system dysfunctions; and septic shock was defined as the subset with cardiovascular dysfunction, which included hypotension, treatment with a vasoactive medication, or impaired perfusion. However, studies that defined sepsis as severe infection leading to life-threatening organ dysfunction were included even if criteria used to define sepsis deviated from the 2005 consensus definitions.

For the purposes of these guidelines, we define septic shock in children as severe infection leading to cardiovascular dysfunction (including hypotension, need for treatment with a vasoactive medication, or impaired perfusion) and "sepsis-associated organ dysfunction" in children as severe infection leading to cardiovascular and/ or noncardiovascular organ dysfunction. Because several methods to identify acute organ dysfunction in children are currently available $[17,19,20,22,23]$, we chose not to require a specific definition or scheme for this purpose.

\section{Scope of patients}

The panel intended these guidelines to apply to all patients from greater than or equal to 37 weeks gestation at birth to 18 years old with severe sepsis or septic shock as defined by the 2005 International Pediatric Sepsis Consensus Conference or inclusive of severe infection leading to life-threatening organ dysfunction. Practically, all infants, children, and adolescents with septic shock or other sepsis-associated acute organ dysfunction are included in this scope. For simplicity, we will henceforth use the term "children" to refer to infants, school-aged children, and adolescents in these guidelines.

All recommendations apply to children with septic shock and other sepsis-associated acute organ dysfunction unless specific qualifications, such as the subset with immune compromise, are included in the recommendation. Even though these guidelines are not intended to address the management of infection with or without SIRS when there is not associated acute organ dysfunction, we recognize that sepsis exists as a spectrum and some children without known acute organ dysfunction may still benefit from similar therapies as those with known organ dysfunction. Finally, acknowledging that neonatal sepsis, especially in premature babies, may have distinct pathology, biology, and therapeutic considerations, newborns less than 37 weeks gestation are excluded from the scope of these guidelines. The panel sought to include term neonates ( $0-28$ days) born at greater than or equal to 37 weeks gestation within the scope of these guidelines because these infants may be recognized and resuscitated outside of a newborn nursery or neonatal ICU. However, because the panel did not specifically address studies of neonates with perinatal infection or conditions that can be associated with neonatal sepsis (e.g., persistent pulmonary hypertension of the newborn), these guidelines do not address all management considerations for neonatal sepsis.

\section{Application of guidelines by local resource availability}

The intended target users of these guidelines are health professionals caring for children with septic shock or other sepsis-associated organ dysfunction in a hospital, emergency, or other acute care setting. However, we acknowledge that many of the recommendations are likely to apply to the care of children with septic shock and other sepsis-associated organ dysfunction across a broad array of settings with adaptation to specific environments and resource availability. 
These guidelines were largely developed without consideration of healthcare resources (with some specific exceptions, e.g., fluid resuscitation), although we realize that medical care for children with septic shock and other sepsis-associated organ dysfunction is necessarily carried out within the confines of locally available resources. The panel supports that these guidelines should constitute a general scheme of "best practice," but that translation to treatment algorithms or bundles and standards of care will need to account for variation in the availability of local healthcare resources. The panel acknowledges as well the need for future research to test the adaptation of interventions to locally available resources.

\section{Funding and sponsorship}

All funding for the development of these guidelines was provided by SCCM and ESICM. In addition, sponsoring organizations provided support for their members' involvement.

\section{Selection and organization of panel members}

The selection of panel members was based on their expertise in specific aspects of pediatric sepsis. Cochairs and co-vice chairs were appointed by the SCCM and ESICM governing bodies; panel members were then recommended by the co-chairs and co-vice chairs. Each panel member was required to be a practicing healthcare professional with a focus on the acute and/or emergent care of critically ill children with septic shock or other sepsis-associated acute organ dysfunction. Broad international and multiprofessional representation from critical and intensive care medicine, emergency medicine, anesthesiology, neonatology, and infectious disease with inclusion of physicians, nurses, pharmacists, and advanced practice providers as part of the working group was ensured. Three members from the lay public were also included with a role to ensure that patient, family, and caregivers' opinions were considered in prioritizing outcomes and finalizing recommendations that the clinicians proposed during the development process. Panelists were recruited from a wide number of countries and healthcare systems, including representation from resource-limited geographic areas. A demographically diverse panel with regard to sex, race, and geography was assembled. Members were then allocated to specific groups based on their expertise.

The methodology team included trained methodologists from McMaster University in Canada (W.A., K.C.) and New York University in the United States (M.E.N.). The team included methodologists with a health research methodology degree (MSc or $\mathrm{PhD}$ ) and/ or advanced methodology training, all of whom are also practicing intensivists. The methodology team provided methodological guidance and leadership throughout the guideline development process.

\section{Question development and outcome prioritization}

The panel was divided into groups: (1) recognition and management of infection, (2) hemodynamics and resuscitation, (3) ventilation, (4) endocrine and metabolic therapies, and (5) adjunctive therapies. A sixth subgroup was added to review research priorities in pediatric sepsis.

The co-chairs, co-vice chairs, and group heads made initial selections of the topics. We included topics addressed in the 2016 SSC adult guidelines that were relevant to children, as well as other key pediatric topics discussed in previously published guidelines [14-16]. The PICO format, which describes the population (P), intervention $(\mathrm{I})$, control $(\mathrm{C})$, and outcomes $(\mathrm{O})$, was used for all guideline questions. Group heads, panel members, and methodologists reviewed and selected PICO questions considered important to guide care for children with septic shock or other sepsis-associated organ dysfunction. Panel members proposed additional PICO questions of high priority and clinical relevance. For practical reasons, we excluded several issues pertaining to general acute or critical illness that were not specific for sepsis (e.g., head-of-bed positioning during invasive mechanical ventilation) and have been addressed in other guidelines (e.g., Pediatric Acute Lung Injury Consensus Conference [PALICC]) [24]. However, topics with particular relevance to children with septic shock or other sepsis-associated acute organ dysfunction were included in this guideline, even if there was evaluation of similar or overlapping topics in previous publications. The final decision regarding PICO question inclusion was reached by discussion and consensus among the guideline panel leaders with input from panel members and the methodology team in each group.

In adherence with the Grading of Recommendations Assessment, Development, and Evaluation (GRADE) approach, panel members compiled a list of potential outcomes for each PICO question. Subsequently, we electronically surveyed panel members and asked them to rate each outcome on a scale of 1 (not important) to 9 (critically important). We selected only outcomes that were critical (mean of 7 or more) for decision-making, taking a patient's perspective. In addition, we presented all selected outcomes to public members to ask for their input and feedback. The final list of PICO questions is provided in Supplemental Table 1, Supplemental Digital Content 1, http://links.lww.com/PCC/B139.

\section{Search strategy and evidence summation}

For each PICO question, a professional medical librarian formulated the search strategy with input from 
the group heads, panel members, and methodologists. Searches utilized a combination of controlled vocabulary (e.g., "sepsis," "bacterial infections," "critical illness," "intensive care units," "pediatrics," "NICU," "PICU," "emergency service") and key words (e.g., "toxic shock," "blood poisoning," "acute infection," "child") in the core search. Additional controlled vocabulary and key words were incorporated to create separate strategies specific to the question posed. Research design filters (e.g., systematic reviews/meta-analyses, randomized controlled trials, observational studies) were also applied as appropriate. Only English language studies were included. No date restrictions were imposed on the searches, but we removed animal-only and opinion pieces from the results. The medical librarian searched a minimum of two major databases (e.g., Cochrane Library, PubMed/MEDLINE, or Embase) to identify relevant systematic reviews, clinical trials, and observational studies published through May 1, 2017. As this was the inaugural version of these guidelines for children, all publications up through May 1, 2017, were considered. Key studies published after the conclusion of the initial literature search on May 1, 2017, were incorporated into the evidence synthesis if identified by panel members as important and relevant even if they were not part of the initial literature review. We excluded articles published in abstract form, in a language other than English, and those focused solely on preclinical data. Panel members, with input from methodologists, used the Cochrane risk of bias tool to assess the risk of bias of randomized trials [25] and Newcastle-Ottawa Scale to assess risk of bias of nonrandomized studies [26].

When applicable, the methodologists used meta-analytic techniques to generate pooled estimates across two or more studies. For meta-analysis of randomized clinical trials (RCTs), we used random-effects model and inverse variance method to pool estimates across relevant studies. We reported relative risks (RRs) and 95\% CI for binary outcomes, and mean difference (MD) and 95\% CI for continuous outcomes. For observational data, we conducted meta-analyses if all individual studies provided adjusted estimates and included both an intervention and a control arm using a random-effects model and inverse variance method to pool adjusted odds ratio (OR) across relevant studies All analyses were conducted using RevMan software (Review Manager, Version 5.3; Copenhagen, Denmark).

\section{Formulation of recommendations}

The GRADE approach principles guided the assessment of quality of evidence from high to very low based on six domains: (1) risk of bias, (2) inconsistency, (3) indirectness, (4) imprecision, (5) publication bias, and (6) assessment of the balance between benefit and harm, patients' values and preferences, cost and resources, and feasibility and acceptability of the intervention [27]. Methodologists performed initial assessments of quality of evidence and incorporated feedback from panel members to generate final evidence profiles using GRADEpro GDT [28].

The panel initially considered only research focused on pediatric patients using a hierarchy of evidence (Table 1). Studies focusing on children with septic shock and other sepsis-associated organ dysfunction were prioritized, although studies inclusive of more general pediatric populations (e.g., all PICU patients) were considered for some questions on a case-by-case basis. If there were no studies or insufficient data in children with sepsis or general pediatric illness, evidence from studies of adult patients was considered using an a priori framework to determine appropriateness of indirect evidence (Fig. 1). Evidence from adult studies was generally down-graded due to the indirectness of the evidence.

In a series of webinars, methodologists reviewed the relevant data for each PICO question with panel members to formulate initial recommendations. Each of the groups used the Evidence-to-Decision (EtD) framework to facilitate transition from evidence to the final recommendation. The EtD framework ensured that panel members took into consideration not only the quality of evidence and magnitude of effect but also balance between benefits and harms, patients' values and preferences, resources, cost, acceptability, and feasibility [28].

We classified recommendations as strong or weak using the language "We recommend..." or "We suggest...," respectively. We judged a strong recommendation in favor of an intervention to have desirable effects of adherence that will clearly outweigh the undesirable effects. We judged a weak recommendation in favor of an intervention to have desirable consequences of adherence that will probably outweigh the undesirable consequences, but confidence is diminished either because the quality of evidence was low or the benefits and risks were closely balanced. The implications of calling a recommendation strong or weak are shown in Table 2. A strong recommendation does not necessarily imply a standard of care, and circumstances may exist in which a strong recommendation cannot or should not be followed for an individual patient. We permitted strong recommendations "for" an intervention based on low or very low quality of evidence when the intervention had the potential to improve survival and there was low risk for immediate harm. We permitted strong recommendations "against" an intervention based on low or very low quality of evidence when there was uncertain benefit but very likely or certain harm, including high costs [29]. 
Table 1 Determination of the quality of evidence

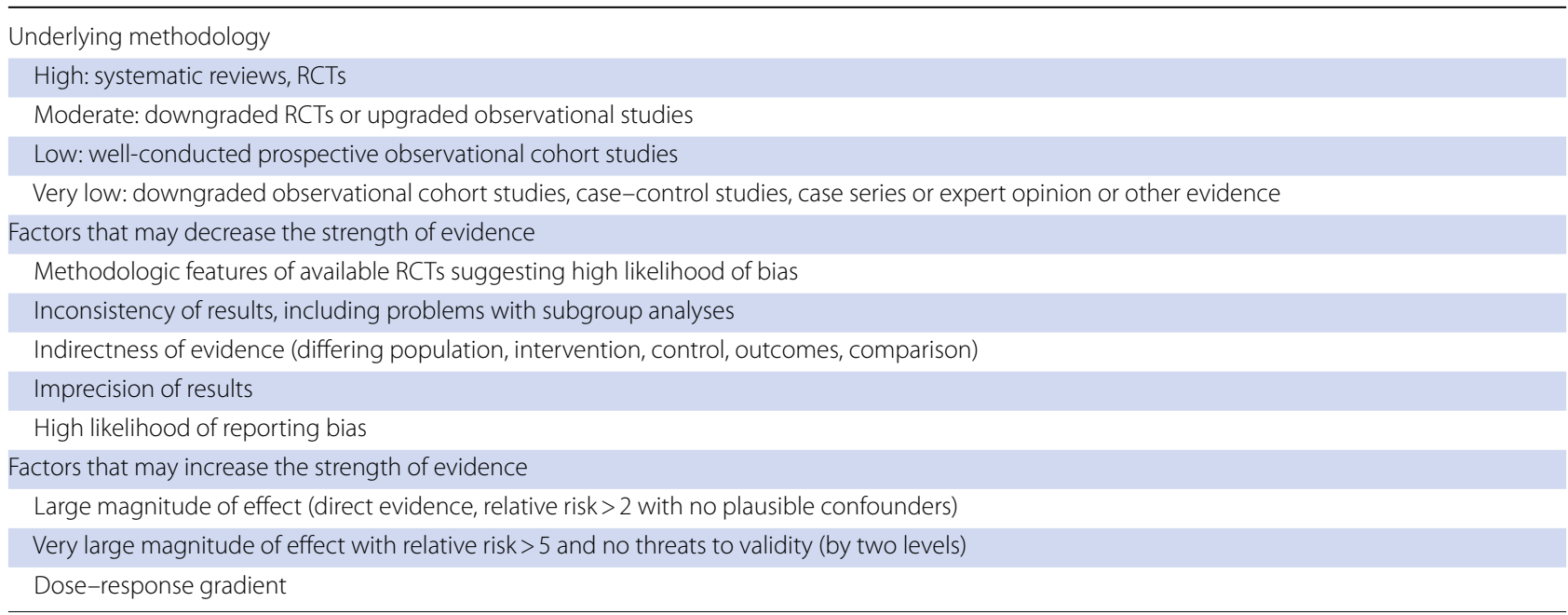

$R C T$ randomized clinical trial

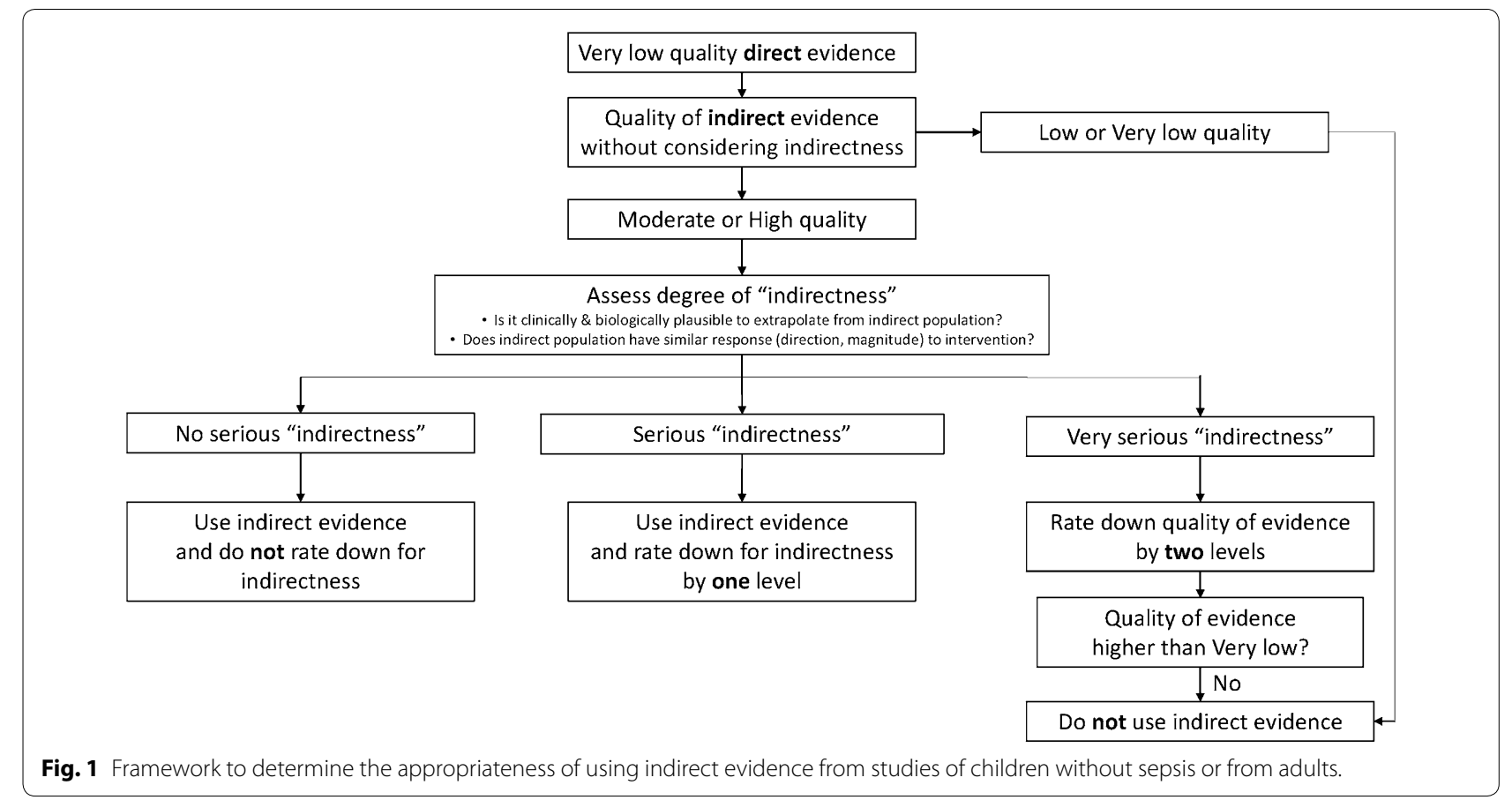

Best practice statements (BPSs) were developed as ungraded strong recommendations within strict conditions suggested by the GRADE Working Group (Table 3) [30]. BPS were issued when the evidence could not be summarized or assessed using GRADE methodology, but the benefit or harm was deemed unequivocal. In addition, when evidence was insufficient to make a recommendation, but the panel felt that some guidance based on current practice patterns may be appropriate, we issued an "in our practice" statement. The "in our practice statements" were developed through a survey of panelists to ascertain their state of current practice. As such, "in our practice" statements are intended only to describe current variation in care and are not meant to be construed as recommendations.

As new data are continuously generated, the SSC is committed to ensuring that these guidelines are updated 
Table 2 Implications of the strength of recommendation

\begin{tabular}{|c|c|c|c|c|c|}
\hline Category & Strength & Quality of evidence & Implications to patients & $\begin{array}{l}\text { Implications to clini- } \\
\text { cians }\end{array}$ & $\begin{array}{l}\text { Implications to policy- } \\
\text { makers }\end{array}$ \\
\hline Strong recommendation & Strong & Usually high or moderate & $\begin{array}{l}\text { Most individuals in this } \\
\text { situation would want } \\
\text { the recommended } \\
\text { course of action, and } \\
\text { only a small proportion } \\
\text { would not }\end{array}$ & $\begin{array}{l}\text { Most individuals should } \\
\text { receive the recom- } \\
\text { mended course of } \\
\text { action. Formal decision } \\
\text { aids are not likely to be } \\
\text { needed to help indi- } \\
\text { viduals make decisions } \\
\text { consistent with their } \\
\text { values and preferences }\end{array}$ & $\begin{array}{l}\text { Can be adapted as policy } \\
\text { in most situations, } \\
\text { including for use as } \\
\text { performance indicators }\end{array}$ \\
\hline Weak recommendation & Weak & Any & $\begin{array}{l}\text { The majority of indi- } \\
\text { viduals in this situation } \\
\text { would want the sug- } \\
\text { gested course of action, } \\
\text { but many would not }\end{array}$ & $\begin{array}{l}\text { Different choices are likely } \\
\text { to be appropriate for } \\
\text { different patients, and } \\
\text { therapy should be tai- } \\
\text { lored to the individual } \\
\text { patient's circumstances, } \\
\text { such as patients'or } \\
\text { family's values and } \\
\text { preferences }\end{array}$ & $\begin{array}{l}\text { Policies will likely be } \\
\text { variable }\end{array}$ \\
\hline Best practice statement & Strong & Ungraded & $\begin{array}{l}\text { Same as strong recom- } \\
\text { mendation }\end{array}$ & $\begin{array}{l}\text { Same as strong recom- } \\
\text { mendation }\end{array}$ & $\begin{array}{l}\text { Same as strong recom- } \\
\text { mendation }\end{array}$ \\
\hline In our practice statement & $\begin{array}{l}\text { Not a rec- } \\
\text { ommen- } \\
\text { dation }\end{array}$ & NA & $\mathrm{NA}$ & $\mathrm{NA}$ & NA \\
\hline
\end{tabular}

NA not applicable

\section{Table 3 Criteria for best practice statement}

Criteria for best practice statement

(1) Is the statement clear and actionable?
(2) Is the message necessary?
(3) Is the net benefit (or harm) unequivocal?
(4) Is the evidence difficult to collect and summarize?
(5) Is the rationale explicit?
(6) Is this better to be formally GRADEd?

Modified from Guyatt et al. [30]

or affirmed every 4 years or sooner if breaking and relevant evidence becomes available.

\section{Voting process}

Panel members convened to review evidence and discuss recommendations in-person and through web conferences. Following the formulation of initial recommendations through discussion within subgroups, all panelists received links to evidence profiles and polls created using SurveyMonkey (Palo Alto, CA) to indicate agreement, disagreement, or abstention. Only panel members without relevant conflicts of interest could vote. Voters could provide feedback for consideration in revising statements. Panelists also deliberated during face-to-face meetings, during which subgroups presented their draft statements for discussion. Up to three rounds of voting were conducted throughout this process of deliberation in an attempt to achieve final consensus. Acceptance of a statement required votes from $75 \%$ of the panel members with an $80 \%$ agreement threshold.

A summary of all statements determined by the panel is shown in "Appendix". Evidence summaries and evidence profiles that informed the recommendations are included in the supplemental tables and figures (Supplemental Digital Content 1, http://links.lww.com/PCC/ B139). Links to specific tables and figures appear within the relevant text.

\section{Conflict of interest policy}

Conflict-of-interest (COI) disclosures were sought through the SCCM from all panelists and support personnel prior to commencing activities, with updates annually and as needed. The process relied solely on personal disclosure, with clarifications sought when necessary, and centered primarily around potential financial conflicts. The co-vice chairs reviewed all COI disclosures in accordance with SCCM's standard operating procedures, sought clarification when necessary, and worked with the co-chairs to recommend appropriate recusals. There was no industry input into or support of the guideline development process. No panelists received honoraria for any role in the guidelines process. Only librarians and a supporting project manager received compensation for their work. 
Seven individuals were identified with potential COIs, but only three were deemed relevant to the final list of questions included in the scope of this guideline. These individuals were asked to abstain from voting on the final recommendations involving the potential COI. In addition, panel members were asked to voluntarily abstain from voting on final recommendations if they had a potential academic COI (e.g., grant application that could benefit from wording of a particular recommendation), although all panel members were welcome to participate in the group discussions leading up to the final recommendation to ensure that input was available from relevant experts.

\section{Screening, diagnosis, and systematic management of sepsis}

1. In children who present as acutely unwell, we suggest implementing systematic screening for timely recognition of septic shock and other sepsis-associated organ dysfunction (weak recommendation, very low quality of evidence).

Remarks: Systematic screening needs to be tailored to the type of patients, resources, and procedures within each institution. Evaluation for the effectiveness and sustainability of screening should be incorporated as part of this process.

Rationale: Systematic screening for sepsis in children is driven by the premise that earlier recognition will lead to more timely initiation of therapy, which will translate to improved morbidity and/or mortality. Screening tools are designed to increase reliability of sepsis recognition and empower health-care professionals to seek rapid medical review. Rapid recognition of sepsis through standardized screening and procedures to guide management of patients identified as at-risk for sepsis should be an essential component of sepsis quality improvement (QI) programs. Although the optimal method or tool for screening is unclear, we suggest that screening tools be adapted to the type of patients, resources, and processes within each institution.

Several studies demonstrating that institutional sepsis QI efforts improve outcomes have successfully incorporated screening tools [31-37]. Most reported sepsis screens were designed to prompt clinicians to prioritize review of patients that had triggered the screen, hence the ultimate decision to treat or not remains with the clinician. Although RCTs have evaluated the role of systematic screening algorithms to recognize clinical deterioration in children more generally [38], high-quality trials on pediatric sepsis recognition are lacking [39], and data are not sufficient to suggest any particular screening tool, although several have been published [40-42] or shared online (http://www.survivingsepsis.org/Resources/Pages /Protocols-and-Checklists.aspx). Single-institution studies demonstrate that an electronic health record (EHR)based screening tool can yield high sensitivity and, when coupled with sequential clinician assessment, improved specificity [43]. For facilities that use an EHR, a stepwise approach combining EHR-triggered alerts followed by clinician assessment has the potential to shorten the time to sepsis recognition [41]. Notably, no study was found on systematic sepsis screening in low- and middleincome countries meeting the PICO criteria.

Institutions should monitor and evaluate their practice following implementation of sepsis screening [44]. Robust QI balancing measures that should be assessed include clinician response, anchoring bias, increased and/or inappropriate antimicrobial prescriptions, fluid overload, increased PICU admissions and transfers to higher levels of care, and healthcare utilization costs [45]. Application of a screening tool requires ongoing optimization of sensitivity and specificity, continuous improvement efforts to maintain provider education and familiarity with the tool, and continual data acquisition to monitor implementation and increase utilization [42]. Finally, screening tools must work well with existing or planned other early warning and rapid response systems $[46,47]$ that may also have inherent limitations $[38,48]$.

2. We were unable to issue a recommendation about using blood lactate values to stratify children with suspected septic shock or other sepsis-associated organ dysfunction into low-versus high-risk of having septic shock or sepsis. However, in our practice, if lactate levels can be rapidly obtained, we often measure blood lactate in children when evaluating for septic shock and other sepsis-associated organ dysfunction.

Rationale: Blood lactate levels provide a valuable indirect marker of tissue hypoperfusion [49]. Although increased lactate levels are not specific, they provide a quantifiable surrogate for tissue hypoxia and can be rapidly obtained by point-of-care tests available in many settings. In adults, blood lactate greater than $2 \mathrm{mmol} / \mathrm{L}$ is now included within the operational definition of septic shock as an indication of cellular/metabolic dysfunction, and measurement of lactate is included in the Hour-1 Sepsis Bundle, with recommendations to repeat lactate measurement if the initial value exceeds $2 \mathrm{mmol} / \mathrm{L}[18$, $50,51]$. In children, several observational studies have demonstrated an association of elevated blood lactate levels with adverse outcomes in septic shock [11, 52-54]. However, the optimal threshold to define "hyperlactatemia" remains unclear. In a PICU study, the mortality 
rate for children with hypotension requiring vasopressors with lactate greater than $2 \mathrm{mmol} / \mathrm{L}$ was $32.0 \%$ compared with $16.1 \%$ if lactate was less than or equal to $2 \mathrm{mmol} / \mathrm{L}$ [11]. Other studies have shown that lactate levels greater than $4 \mathrm{mmol} / \mathrm{L}$ are consistently associated with mortality [52]. Although blood lactate may be affected by the conditions of the blood draw (e.g., use of a tourniquet), both venous and arterial lactate measurements obtained have been shown to be independently associated with mortality in children [55]. In one prospective study in children, normalization of lactate within $2-4 \mathrm{~h}$ of presentation was associated with decreased risk of persistent organ dysfunction (adjusted RR, 0.47; 95\% CI, 0.29-0.78) [56]. However, no RCTs have tested whether initial or serial measurement of blood lactate directly informs evaluation and/or management in children. Lactate levels should therefore be interpreted as part of a more comprehensive assessment of clinical status and perfusion.

3. We recommend implementing a protocol/guideline for management of children with septic shock or other sepsis-associated organ dysfunction (BPS).

Rationale: Institutional protocols have been shown to improve the speed and reliability of care for children with septic shock or other sepsis-associated organ dysfunction. Studies reported improvements in mortality, length of stay (LOS), duration of organ dysfunction, and development of new or progressive multiple organ dysfunction syndrome [8, 32-34, 36, 57-61]. Most of these studies have focused on timely delivery of a "bundle of therapies" (e.g., blood culture, fluid bolus, and antibiotics). For example, an analysis of 1179 children with sepsis across 54 hospitals in New York State found that completion of a sepsis bundle within $1 \mathrm{~h}$ was associated with lower risk-adjusted OR of in-hospital mortality $(0.59 ; 95 \% \mathrm{CI}$, $0.38-0.93 ; \mathrm{p}=0.02$ ) [8]. In a recent single-institution study, bundle-compliant care in 1380 children with septic shock was associated with a five-times lower mortality (OR, 0.20; 95\% CI, 0.07-0.53) [33]. In another study, implementation of a sepsis protocol led to a substantial increase in the proportion of children who no longer had organ dysfunction on day 2 after presentation (adjusted OR, 4.2; 95\% CI, 1.7-10.4) [34]. However, it should be noted that protocols studied to date have variable components, many studies do not report adherence to specific items within protocols, and only a few studies have attempted to adjust for initial illness severity or other patient factors, making it difficult to summarize studies using the GRADE approach. Therefore, because available evidence shows a strong and consistent association that adherence to protocols reduces variability in care and improves outcomes, we recommend implementing a protocol/guideline for management of children with septic shock or other sepsis-associated organ dysfunction as a best practice.

\section{We recommend obtaining blood cultures before initiat- ing antimicrobial therapy in situations where this does not substantially delay antimicrobial administration (BPS).}

Rationale: Blood cultures remain the most commonly used method to identify bacteremia. Identification of a blood-borne pathogen can have significant clinical implications on the type and duration of antimicrobial therapy and is an important mechanism to recognize multidrugresistant pathogens [62]. Thus, whenever possible, blood cultures should be obtained prior to initiation of antimicrobial therapy in children with severe sepsis or septic shock. Although no studies have directly measured the effect of blood cultures alone on outcome in pediatric sepsis, several observational studies have demonstrated that a bundled approach to initial resuscitation that includes early blood cultures is associated with improved outcomes [8, 31, 33]. If collection of the blood cultures is likely to delay administration of antimicrobial therapy to the patient, then administration of antimicrobials should take precedence, in view of the impact of delayed antimicrobial administration on patient outcomes [63]. However, because blood cultures may be the only source of information identifying bacterial antibiotic susceptibility, it is important to make all reasonable efforts to collect blood cultures before timely antimicrobial administration. The collection of other biological specimens to identify pathogens from nonblood sites (e.g., urine, cerebrospinal fluid, tracheal aspirate, bronchoalveolar lavage, drainage from collections) should also happen as soon as possible, and depending on the suspected site of infection, such specimens may have a higher yield of pathogen identification than blood cultures. Clinicians should also consider the epidemiology of pediatric infections in relation to age, sex, and host factors, such as comorbidities $[64,65]$. Specific patterns of pediatric bloodstream infections relating to age and comorbidities are well known, and approximatively one out of three bacteremia episodes are associated with organ dysfunction in a recent large population-based study [65].

Limitations of standard blood cultures include the time needed to grow and then identify pathogens and their antibiotic sensitivities, as well as the effect of previous therapy on diagnostic yield. New molecular technologies are becoming available to facilitate earlier and faster microbiological diagnoses. Such techniques may be able to identify a range of pathogens well before blood cultures are positive [66], and may potentially identify 
pathogens even after the administration of antimicrobial therapy. However, new molecular diagnostics are currently relatively expensive, are not sufficient for all pathogens and antibiotic sensitivities, and are not universally available.

\section{Antimicrobial therapy}

5. In children with septic shock, we recommend starting antimicrobial therapy as soon as possible, within $1 \mathrm{~h}$ of recognition (strong recommendation, very low quality of evidence).

6. In children with sepsis-associated organ dysfunction but without shock, we suggest starting antimicrobial therapy as soon as possible after appropriate evaluation, within 3 h of recognition (weak recommendation, very low quality of evidence).

Rationale: Antimicrobials are the primary medical therapy that directly targets the underlying cause of sepsis, and there is strong biologic rationale for rapid delivery of antimicrobials in patients with sepsis [44]. Many QI initiatives have shown improved pediatric sepsis outcomes with implementation of a bundle that includes rapid delivery of IV antimicrobials [8, 32-34, 36, 57-61]. Two retrospective observational studies have also demonstrated an association of faster time to antimicrobial therapy with reduced mortality for children with sepsis. The first study was an analysis of 130 children with sepsis (mortality of $12 \%)$, including 103 (79\%) with septic shock, in which the unadjusted OR for mortality among children with antimicrobials delivered within versus after 60 min of sepsis recognition was 0.60 (95\% CI, 0.13-2.86) [63]. The second study was an analysis of 1179 children, including 69\% with septic shock, where completion of a sepsis bundle within $1 \mathrm{~h}$ of sepsis recognition was associated with decreased mortality (OR, 0.59; 95\% CI, 0.38-0.93; $\mathrm{p}=0.02$ ); however, initiation of antimicrobials alone by $1 \mathrm{~h}$ of recognition was not associated with significant mortality reduction (OR, 0.78; 95\% CI, 0.55-1.12; $\mathrm{p}=0.18$ ) [8]. When the adjusted OR of these two studies were pooled, there was a possible reduction in mortality (OR, 0.77; 95\% CI, 0.55-1.08) (Supplemental Table 2 and Supplemental Fig. 1, Supplemental Digital Content 1, http://links.lww.com/PCC/B139). Other secondary endpoints reported in the literature have also been associated with shorter time to initiation of antimicrobial therapy, including reduced LOS, shorter duration of organ dysfunction, and reduced development of new or progressive multiple organ dysfunction syndrome [8, 32-34, 36, 57-61]. Furthermore, indirect evidence from adult sepsis generally supports a benefit to starting antimicrobial therapy as soon as possible after recognition of septic shock [67-72]. Thus, timely antimicrobial therapy-ideally administered as part of a more comprehensive bundle of initial care-should be the goal for children with septic shock.

The definition of "timely" in this context represents an area of controversy relating to challenges in the accurate recognition of patients with sepsis and septic shock and the need to consider balancing QI metrics such as unnecessary antimicrobial usage $[44,73,74]$. One pediatric study [63] indicated a dose-response gradient such that the longer time to antimicrobial therapy, the higher the mortality. Yet the mortality increase reached significance only when antimicrobials were administered greater than $3 \mathrm{~h}$ in comparison to less than $3 \mathrm{~h}$, whereas the mortality of patients receiving antimicrobials within less than $1 \mathrm{~h}$ was not different from those receiving antimicrobials within less than $3 \mathrm{~h}$ in that relatively small study. The second, larger pediatric study demonstrated a significant decrease in mortality if antimicrobials were administered within $1 \mathrm{~h}$, but only in the context of a bundle that included a blood culture and fluid bolus [8]. Thus, available pediatric studies do not provide a clear time cutoff after which the risk of mortality or other adverse outcomes increases, but rather support that there is likely to be an incremental risk for harm as time to antimicrobial initiation increases, in particular beyond $3 \mathrm{~h}$. Notably, the benefit of antimicrobial therapy within $1 \mathrm{~h}$ of recognition has been most prominent in cohorts with a predominance of septic shock (as compared with sepsis without shock) patients $[8,63]$.

Based on limited pediatric evidence and indirect evidence from adult studies, the panel supported that, in children "with septic shock," antimicrobial therapy should be initiated as soon as possible and ideally within $1 \mathrm{~h}$ of recognition. Suspicion of septic shock can usually be guided by clinical findings rapidly ascertained through history and physical examination. Although our recommendation to ideally administer antimicrobial administration within $1 \mathrm{~h}$ of recognition of septic shock establishes a tangible goal that emphasizes the importance of early antimicrobial therapy and assists clinicians in prioritizing bedside care, this cut-point should not be misconstrued as a known biological truth. Thus, dichotomous time-based metrics of the quality of care for children with sepsis, while pragmatic and potentially useful to trend, may be of less value than use of continuous variables such as median time to antimicrobials. Despite a very low quality of evidence on this topic, we provide a strong recommendation because the panel concluded that most patients would accept and most clinicians should seek to initiate antimicrobial therapy as soon as possible after recognition of septic shock in most situations. 
For children "without clinical signs of shock," the panel acknowledged that the diagnosis of sepsis-associated organ dysfunction has additional challenges related to the need to discriminate those with true sepsis from among a large number presenting with suspected infection [44]. In view of the available evidence, we suggest starting antimicrobial therapy as soon as possible after sepsis recognition, while allowing up to $3 \mathrm{~h}$ for appropriate diagnostic investigation for patients without clinical signs of shock and for those with an uncertain diagnosis. However, the diagnostic evaluation should be performed expeditiously and, if and when the evaluation supports a likely infection or evidence of septic shock or other sepsis-associated organ dysfunction becomes manifest, antimicrobial therapy should be immediately administered.

7. We recommend empiric broad-spectrum therapy with one or more antimicrobials to cover all likely pathogens (BPS).

8. Once the pathogen(s) and sensitivities are available, we recommend narrowing empiric antimicrobial therapy coverage (BPS).

9. If no pathogen is identified, we recommend narrowing or stopping empiric antimicrobial therapy according to clinical presentation, site of infection, host risk factors, and adequacy of clinical improvement in discussion with infectious disease and/or microbiological expert advice (BPS).
Rationale: Sepsis mortality is associated with delays to "appropriate" antimicrobial therapy, and hence optimal treatment for sepsis relies on accurate selection of antimicrobials to ensure activity against the major pathogens [50, 63, 70, 75]. "Empiric therapy" refers to the initial choice of antimicrobials pending microbiological results (Table 4) and is based on the predicted likelihood of bacterial pathogens. Empiric therapy should cover a broad range of pathogens that are likely to cause the infection, acknowledging that, in rare circumstances, this may not fully cover very unusual pathogens. "Broad-spectrum therapy" refers to the use of "single- or multi-drug" antimicrobial therapy with activity against multiple groups of bacteria/pathogens. Broad-spectrum therapy is recommended for initial empiric therapy of children with septic shock or sepsisassociated organ dysfunction to increase the likelihood that the initial empirical therapy is effective against the causative pathogens.

The initial choice of empiric antimicrobials should take into account the specific clinical history (e.g., age, site of infection, concomitant disease states, comorbid conditions, indwelling devices). Patients with recent or current hospital exposure should receive empiric therapy that considers known infection or colonization, as well as any recent antimicrobial exposure. Institutions or regions should identify the most appropriate first-line singleagent antimicrobial, taking into account anatomic site of infection, age, local epidemiology, and host comorbidity and risk factors (e.g., ceftriaxone is recommended for

Table 4 Definitions for empiric, targeted/definitive, broad-spectrum, and multiple drug antimicrobial therapy

\begin{tabular}{|c|c|c|}
\hline Term & Definition & Comment \\
\hline Empiric antimicrobial therapy & $\begin{array}{l}\text { Initial antimicrobial therapy started for suspected } \\
\text { infection in the absence of definitive microbio- } \\
\text { logic pathogen identification }\end{array}$ & $\begin{array}{l}\text { Empiric therapy may consist of single or multiple } \\
\text { agents but should be broad spectrum in nature. } \\
\text { Empiric antimicrobial therapy should be based on } \\
\text { local, regional, or national pathogen epidemiology } \\
\text { and patient risk factors }\end{array}$ \\
\hline Targeted/definitive antimicrobial therapy & $\begin{array}{l}\text { Antimicrobial therapy targeted to specific } \\
\text { pathogen(s), usually after microbiologic identifica- } \\
\text { tion }\end{array}$ & $\begin{array}{l}\text { Targeted/definitive therapy may consist of single or } \\
\text { multiple agents, but should not be broader than } \\
\text { required to treat the specific pathogen(s) after } \\
\text { microbiologic identification }\end{array}$ \\
\hline Broad-spectrum antimicrobial therapy & $\begin{array}{l}\text { An antimicrobial regimen with activity against } \\
\text { multiple different groups of bacteria or other } \\
\text { pathogens considered to be likely causes of the } \\
\text { clinical presentation }\end{array}$ & $\begin{array}{l}\text { Broad-spectrum antimicrobial therapy may consist of } \\
\text { single or multiple agents }\end{array}$ \\
\hline Multiple-drug antimicrobial therapy & $\begin{array}{l}\text { More than one antimicrobial agent is needed to } \\
\text { either (1) expand the spectrum of coverage to } \\
\text { include additional pathogens (e.g., vancomycin } \\
\text { for methicillin-resistant Staphyloccocus aureus; } \\
\text { (2) decrease the likelihood of resistance to any par- } \\
\text { ticular single agent (e.g., for patients with known } \\
\text { or high-risk for multi-drug resistant organism); or } \\
\text { (3) provide synergy to treat a suspected or known } \\
\text { pathogen }\end{array}$ & \\
\hline
\end{tabular}


community-acquired sepsis by the National Institute for Health and Care Excellence [NICE] in the United Kingdom) [16]. For complex patients or those recently or currently in hospital, the choice of empiric antimicrobials should also take into account concomitant underlying diseases, chronic organ failure, indwelling devices, the presence of immunosuppression or other form of immunocompromise, recent known infection or colonization with specific pathogens, and recent receipt of antimicrobials $[65,76,77]$. When available, an infectious diseases clinician should be consulted. Other nonbacterial pathogens that are suspected as a cause of infection should also be targeted as part of initial antimicrobial therapy on a case-by-case basis.

Sepsis in children is most commonly due to gramnegative or gram-positive bacteria, although the relative prevalence of these pathogens varies by age, geographic region, location (community vs hospital) of sepsis onset, and other patient factors. Invasive fungal infections are largely restricted to immunocompromised patients and preterm infants. Certain specific conditions put patients at risk for atypical or resistant pathogens, thus requiring specific empiric regimens. For example, neutropenic patients are at risk for an especially wide range of potential pathogens, including resistant gram-negative bacilli and Candida species, and neonates are at risk for sepsis caused by Listeria monocytogenes and disseminated herpes simplex virus (HSV). Children with chronic conditions treated in hospital settings are prone to sepsis with resistant bacteria such as methicillin-resistant Staphylococcus aureus (MRSA) and vancomycin-resistant enterococci. For children at risk for multidrug-resistant bacterial infections, empiric broad-spectrum antimicrobial regimens may require more than one agent to broadly cover such potential pathogens.

For specific empiric broad-spectrum antimicrobial therapy, the reader is directed to published resources $[76,78]$ and the need to consider patient history, allergies, local epidemiology, and suspected site/source of infection. However, general suggestions can be provided here. For previously healthy children with communityacquired sepsis, a third-generation cephalosporin (e.g., ceftriaxone) may be sufficient. Vancomycin should be added in settings where MRSA or ceftriaxone-resistant pneumococci are prevalent, and addition of an aminoglycoside or substitution of a carbapenem is appropriate in settings where ceftriaxone resistance is common in gramnegative bacteria [79]. For immunocompromised patients or hospital-acquired sepsis, antimicrobial therapy should begin with an anti-pseudomonal third- or higher-generation cephalosporin (e.g., cefepime), a broad-spectrum carbapenem (e.g., meropenem, imipenem/cilastatin), or an extended-range penicillin/ $\beta$-lactamase inhibitor combination (e.g., piperacillin/tazobactam) [78]. For neonates, therapy should also include ampicillin for listeria and consideration for empiric acyclovir if there is a clinical concern for HSV [76]. For patients with a suspected or documented intra-abdominal source of infection, therapy should include broad coverage for gastrointestinal pathogens, including anaerobic bacteria, with either an extended-range penicillin/ $\beta$-lactamase inhibitor combination or carbapenem, or addition of clindamycin or metronidazole. For patients who present with sepsis complicating an influenza-like illness during the local influenza season, empiric antiviral therapy should be started while awaiting the respiratory virus testing [80, 81]. Patients at higher risk of antibiotic-resistant infection because of past infection or colonization, local epidemiology, or recent broad-spectrum antibiotic use should receive an individually tailored empiric therapeutic regimen [82]. In cases of suspected toxic shock syndrome or necrotizing fasciitis, empiric treatment should include clindamycin or lincomycin to limit toxin production and enhance bacterial clearance [83]. Finally, for sepsis treated in regions endemic for rickettsial or parasitic pathogens (e.g., malaria), clinicians should consider adding relevant empiric coverage.

"Targeted or definitive therapy" refers to the antimicrobial regimen targeted to a specific pathogen(s) after microbiologic identification. As with empiric therapy, targeted/definitive therapy may be single- or multidrug therapy, but should not be broader than required to treat the specific pathogen(s) after microbiologic identification [84, 85]. Risks of unnecessary continuation of broad-spectrum antibiotic and other antimicrobial therapy include direct side effects and toxicities (such as the nephrotoxicity or ototoxicity of aminoglycosides), infection with Clostridioides difficile (formerly Clostridium) or fungal pathogens, and promotion of antimicrobial resistance in the patient and in the community. In addition, unnecessary exposure to antibiotics may lead to alteration of the human microbiome early in life, the impact of which is poorly understood but has been associated with worse outcomes such as necrotizing enterocolitis in newborns.

Because most microbiological cultures show significant growth within 24 to $36 \mathrm{~h}$ of collection when a pathogen is present [86], empiric treatment should be reevaluated after no more than $48 \mathrm{~h}$ following initiation. If no pathogen is identified and bacterial/fungal infection is deemed unlikely, clinicians should stop empiric antimicrobial therapy to reduce unnecessary exposure to antibiotics/antifungals. However, many children with a clinical diagnosis of septic shock do not have a pathogen isolated $[5,6]$. Patients with negative bacterial microbiological results may have false-negative tests 
due to antibiotic pretreatment, absence of bacteremia (e.g., bacterial pneumonia despite true bacterial infection), or sepsis-related to viral infections [87]. Thus, the decision to continue, narrow, or stop antimicrobial therapy must often be made on the basis of clinician judgment and indirect clinical information, taking into account the clinical presentation, site and type of infection, host risk factors, and adequacy of clinical improvement. Complex patients should be discussed with pediatric infectious diseases and/or microbiology specialists to ensure likely pathogens are treated and that antibiotics and other antimicrobials are stopped when they are no longer necessary.

\section{In children without immune compromise and without} high risk for multidrug-resistant pathogens, we suggest against the routine use of empiric multiple antimicrobials directed against the same pathogen for the purpose of synergy (weak recommendation, very low quality of evidence).

Remarks: In certain situations, such as confirmed or strongly suspected group B streptococcal sepsis, use of empiric multiple antimicrobials directed against the same pathogen for the purpose of synergy may be indicated.

11. In children with immune compromise and/or at high risk for multidrug-resistant pathogens, we suggest using empiric multi-drug therapy when septic shock or other sepsis-associated organ dysfunction is present/suspected (weak recommendation, very low quality of evidence).

Rationale: The selection of an empiric antimicrobial regimen requires consideration of a patient's underlying disease state, potential history of prior infections and colonization with multidrug-resistant organisms, presence of immunosuppression, and possible recent antimicrobial use, as well as local pathogen prevalence and susceptibility profile $[50,88,89]$. Empiric therapy may be single- or multi-drug, but should be broad spectrum in nature as defined in Table 4. For select patients or with concern for particular types of infection, this may necessitate adding a glycopeptide (i.e., vancomycin) to ensure empiric coverage of MRSA or a second gram-negative agent (e.g., aminoglycoside in addition to a beta-lactam or second/third-generation cephalosporin) when antibiotic resistance is a concern. However, routinely including an aminoglycoside or a glycopeptide for synergy or "double-coverage" as part of an empiric regimen is not supported by the available data [89-100].

A recent Cochrane review evaluated beta-lactam monotherapy versus beta-lactam and aminoglycoside combination regimens for sepsis and included 69 trials accounting for 7863 participants, including neonatal and pediatric patients [88]. In trials where the mono- and multidrug arm used the same beta-lactam, no difference in clinical outcomes was observed between study groups. In studies where the monotherapy arm contained a beta-lactam of broader spectrum than the multidrug arm, monotherapy showed a possible benefit for all-cause mortality (OR, 0.85; 95\% CI, 0.71-1.01) and a significant advantage for clinical failure (OR, 0.75; 95\% CI, 0.67-0.84) [88]. Additionally, indirect evidence from adults with sepsis including 13 RCTs comparing empirical mono- versus combination antibiotic therapy suggests mortality and other outcomes are not improved by empiric combination therapy [90]. Therefore, many children with septic shock and other sepsis-associated organ dysfunction do not require empiric multi-drug therapy. Clinicians should continually reevaluate the local epidemiology and resistance rates to ensure monotherapy remains appropriate [88].

Certain clinical scenarios, however, may necessitate multi-drug antimicrobial therapy. For example, in patients at high risk for resistant gram-negative infections with sepsis, combining a beta-lactam/beta-lactamase inhibitor agent (i.e., piperacillin/tazobactam combination) with an aminoglycoside (i.e., gentamicin) can be considered, not for synergy, but for expanded coverage to treat both susceptible and resistant pathogens until final identification and susceptibilities are known [101-103]. Additionally, a synergistic multi-drug regimen may be appropriate in select settings, even for targeted/ definitive therapy, such as device-associated infections, enterococcal endocarditis, staphylococcal endocarditis, group B streptococcal sepsis, and carbapenem-resistant Enterobacteriaceae infections $[104,105]$.

Pediatric patients with cancer and transplant recipients have a substantial degree of immunosuppression and represent a population at higher risk for colonization and infection with multi-drug resistant organisms [106, 107]. The 2017 guidelines for the management of fever and neutropenia (FN) in children with cancer and hematopoietic stem-cell transplantation recommended monotherapy with an anti-pseudomonas beta-lactam, a fourth-generation cephalosporin, or a carbapenem as empiric therapy in high-risk pediatric patients with FN [78]. The three RCTs in high-risk pediatric FN comparing monotherapy with aminoglycoside-containing combination therapy found no significant differences in failure rates, infection-related mortality, or overall mortality $[78,108,109]$. The meta-analysis also confirmed the efficacy and safety of monotherapy without the addition of an aminoglycoside. However, the 2017 guidelines on the management of children with FN did recommend 
addition of a second gram-negative agent and/or a glycopeptide when resistant organisms were suspected for patients who are clinically unstable (i.e., septic shock) and in centers with a high rate of resistant pathogens [78]. Therefore, for children with septic shock or other sepsisassociated organ dysfunction who have immune compromise and/or are at high risk for multidrug-resistant pathogens, we suggest empiric multi-drug therapy.

Currently, specific resistance rate thresholds do not exist to help clinicians decide when the addition of a glycopeptide or second gram-negative agent for sepsis or septic shock is necessary. The U.S. guidelines for the management of community-acquired pneumonia in adults suggest a $25 \%$ rate of high-level macrolide resistance in the community as the threshold beyond which macrolides should not be used [110, 111]. Additionally, current guidelines from the Infectious Diseases Society of America recommend an alternative antibiotic for skin and soft tissue infections if the local clindamycin resistance rate is greater than $10 \%$ [112]. Considering the current rates of morbidity and mortality for patients with sepsis or septic shock, a local or regional antimicrobial resistance rate exceeding $10 \%$ is probably a prudent threshold for the addition of a second agent if that pathogen is suspected $[5,63]$.

12. We recommend using antimicrobial dosing strategies that have been optimized based on published pharmacokinetic/pharmacodynamic principles and with consideration of specific drug properties (BPS).

Rationale: Sepsis may alter the pharmacokinetics and pharmacodynamics of antimicrobials. Therefore, antimicrobial dosing should be individualized to deliver effective and timely treatment of life-threatening infection, while at the same time limiting adverse medication effects. Sub-therapeutic dosing can lead to failure to clear the infection, prolong organ dysfunction, and can lead to the development of antimicrobial resistance. A substantial proportion of sepsis patients are at risk for altered drug metabolism and/or clearance, including those with kidney and hepatic dysfunction and those treated with extracorporeal therapies [113]. In particular, continuous renal replacement therapy (CRRT) and extracorporeal membrane oxygenation (ECMO) both lead to profound alteration of antimicrobial clearance, requiring individual dose adaptation [114]. Therapeutic drug monitoring, where available, can permit individualized antimicrobial dosing to achieve maximal effect while minimizing toxicity [115].

Examples of sepsis and septic shock-related altered pharmacokinetics include increased volume of distribution as a result of fluid therapy and capillary leak [116], decreased antimicrobial clearance as a result of altered renal and hepatic organ perfusion and organ dysfunction [117], and higher unbound drug levels due to hypoalbuminemia leading to increased clearance [118]. Hepatic dysfunction impairs the metabolism of lipophilic and highly albumin-bound antibiotics, leading to drug accumulation and toxicity. In renal dysfunction, timedependent antibiotics cleared by the kidneys, such as the beta-lactams, require reduced dosing frequency.

The three main determinants of antimicrobial efficacy are (1) the time during which the concentration of the drug remains above the minimum inhibitory concentration (MIC) of the causative pathogen $(\mathrm{T}>\mathrm{MIC})$ (time-dependent antibiotics); (2) the peak concentration to MIC ratio (Cmax/MIC) (concentration-dependent antibiotics); and (3) the ratio of the 24-h area under the concentration-time curve divided by the MIC (AUC24/ MIC) (concentration-dependent with time-dependence antibiotics). The main classes of time-dependent antibiotics include beta-lactams (penicillins, cephalosporins, carbapenems, monobactams) and lincosamides (clindamycin and lincomycin). For amoxicillin-clavulanic acid, current published dosing regimens in critically ill children can result in sub-therapeutic concentrations in the early period of sepsis due to augmented renal clearance $[119,120]$. In sepsis, the use of continuous or extended infusions with loading doses, as opposed to intermittent dosing, may lead to improved outcomes in patients treated with beta-lactam antibiotics [121].

The main classes of concentration-dependent antibiotics include aminoglycosides and metronidazole. In some centers, drug concentrations measured within $60 \mathrm{~min}$ before or after administration of aminoglycosides are used to estimate the Cmin and Cmax, respectively, and together with the MIC of the pathogen, can help to guide appropriate antimicrobial dosing [118]. Concentrationdependent antibiotics may require an altered dosing frequency to maximize bacterial killing by preserving the Cmax/MIC.

Glycopeptides, oxazolidinones, fluoroquinolones, polymixins, daptomycin, azithromycin, and tigecycline are examples of concentration-dependent with time-dependent antibiotics. For vancomycin, this can mean higher doses, but that comes with an increased risk of toxicity. For this reason, continuous vancomycin infusions may be considered to achieve optimal concentrations in some patients [122]. For concentration-dependent with timedependent antibiotics, dose optimization involves adjusting the dosing interval rather than administered dose [118].

13. In children with septic shock or sepsis-associated organ dysfunction who are receiving antimicrobials, we rec- 
ommend daily assessment (e.g., clinical, laboratory assessment) for de-escalation of antimicrobial therapy (BPS).

Remarks: This assessment should include a review of the ongoing indication for empiric antimicrobial therapy after the first $48 \mathrm{~h}$ that is guided by microbiologic results and in response to clinical improvement and/or evidence of infection resolution. This recommendation applies to patients being treated with empiric, targeted, and combination therapy.

Rationale: The misuse and overuse of broad-spectrum antimicrobials in healthcare, the community, veterinary medicine, and the environment have contributed to a global public health emergency [123]. De-escalation of antimicrobials, where appropriate, is warranted to minimize adverse effects of unnecessarily prolonged administration. To date, QI efforts in adults have shown that safe and effective antimicrobial de-escalation can be achieved by daily assessment and discussion [124, 125].

Several host biomarkers have also been proposed to aid in the safe de-escalation of antimicrobial therapy. In adults with severe infections and sepsis, procalcitonin has been shown to successfully guide de-escalation [126-130] with an associated improved mortality [131]. Similar reductions in length of antimicrobial therapy have also been safely achieved in neonatal populations [132] using procalcitonin as a guide. In the United Kingdom, the NICE committee concluded that in emergency department and critical care settings, procalcitonin testing shows promise, but currently, insufficient evidence is available to recommend the routine adoption of procalcitonin-guided antimicrobial de-escalation (www.nice.org. uk/guidance/dg18).

Although a relationship between antimicrobial stewardship programs (ASPs) and a decrease in antimicrobial resistance has not yet been shown, studies suggest that inpatient pediatric ASPs may reduce antimicrobial usage without contributing to adverse patient outcomes [123]. The "Start Smart - Then Focus" work from Public Health England suggests a pragmatic approach of the five "antimicrobial prescribing decision" options to include (1) stop antimicrobials if there is no evidence of infection, (2) switch antimicrobials from IV to oral, (3) change antimicrobials-ideally to a narrower spectrum-or broader if required, (4) continue and document next review date or stop date, and (5) outpatient parenteral antimicrobial therapy [133]. De-escalating antimicrobial therapy must be based in sound clinical judgment and needs to be adapted to local epidemiology and identified resistance patterns.
14. We recommend determining the duration of antimicrobial therapy according to the site of infection, microbial etiology, response to treatment, and ability to achieve source control (BPS).

Rationale: The main purposes of antimicrobial therapy in patients with sepsis are to reduce the pathogen load rapidly and to prevent recurrence. Important determinants of the required duration of antimicrobial therapy include site of infection, ability to drain or remove fixed infectious foci, choice of antimicrobial therapy, time to clearance of positive cultures, the nature of the causative pathogen, and the integrity of the host immune response. There is no evidence that severity of sepsis is an important determinant of optimal duration of therapy because illness severity is not expected to affect clearance of infection.

The optimal duration of antimicrobial therapy can differ by site of infection because of a high pathogen burden, poor antimicrobial penetration, or presence of difficult-to-eradicate microbial biofilms at the site. For example, longer duration of therapy is typically required for treatment of endocarditis, undrained abscesses, and prosthetic joint infection without device removal [134136]. Characteristics of the causative organism that may affect optimal duration of therapy include resistance or decreased susceptibility to front-line antimicrobials and propensity to cause deep-seated or difficult-to-eradicate infection. For example, optimal duration of treatment for endocarditis caused by methicillin-susceptible $\mathrm{S}$. aureus may be shorter than for that caused by MRSA [136]. Similarly, although 7-10 days of therapy is appropriate for treatment of uncomplicated gram-negative bacteremia in immunocompetent hosts [137, 138], uncomplicated S. aureus bacteremia requires a longer course of therapy to effect cure [139-141], likely because of unrecognized seeding [142]. Integrity of host immunity may also affect clearance of infection, so antimicrobial therapy for infection in neutropenic pediatric patients with cancer is often continued until resolution of neutropenia [78].

A systematic review evaluated studies describing duration of treatment for clinically and microbiologically-documented infections in children and provides evidence-based clinical guidelines for optimal duration of antimicrobial therapy for specific conditions [143]. Given the lack of studies on the duration of antimicrobial therapy for pediatric patients with sepsis specifically, we refer to this previously published guideline as best evidence. Importantly, there are no data to support that the presence of organ dysfunction or a higher initial illness severity necessitates longer therapy for specific infection types (other than attention to how such organ 
dysfunction may affect antimicrobial pharmacokinetics and pharmacodynamics).

Observational studies suggest that longer exposure to antibiotics is associated with risk of potential adverse events including necrotizing enterocolitis in very low birthweight infants [144], candidemia in hospitalized children [145, 146], development of antimicrobial resistance [147] and C. difficile (formerly Clostridium) infection [148]. Several meta-analyses, RCTs, and observational studies have compared long- versus short-duration antibiotic therapy for serious infections [140, 144, 149-167]. Most studies suggest that shorter courses were associated with similar clinical outcomes compared with longer durations; these include neonatal bacteremia $[158,163]$, pyelonephritis [168], uncomplicated bacterial meningitis [154, 155, 159-161, 164, 165], and pneumonia $[169,170]$. In contrast to these infections, some studies have identified scenarios where longer durations of antimicrobial therapy is superior. For example, an RCT suggested that 14 days of antibiotic therapy was superior to 7 days for treatment of neonates with S. aureus bacteremia [140], and an observational study suggested that greater than 10 days was superior to less than or equal to 10 days of antibiotic therapy in children treated for gramnegative bacteremia without removal of a preexisting central venous catheter (CVC) [162].

\section{Source control}

15. We recommend that emergent source control intervention be implemented as soon possible after a diagnosis of an infection amenable to a source control procedure is made (BPS).

Remarks: Appropriate diagnostic testing to identify the site of infection and microbial etiology should be performed, and advice from specialist teams (e.g., infectious diseases, surgery) should be sought, as appropriate, in order to prioritize interventions needed to achieve source control.

16. We recommend removal of intravascular access devices that are confirmed to be the source of sepsis or septic shock after other vascular access has been established and depending on the pathogen and the risks/ benefits of a surgical procedure (strong recommendation, low quality of evidence).

Rationale: Source control is defined as physical modalities taken to control or remove the source of infection or to prevent spread of the infection systemically or to adjacent tissues [171]. Source control may include percutaneous or deep abscess drainage, drainage of an empyema, septic joint, or subperiosteal abscess, removal of infected hardware or CVCs, or debridement of necrotizing soft-tissue infection. The adult SSC guidelines recommend source control as soon as is reasonably feasible after resuscitation, ideally within 6-12 h of diagnosis [50]. Waiting for patients to clinically stabilize prior to intervention is not recommended, as delaying adequate source control may lead to further clinical deterioration [6]. Although source control as an adjunct to antimicrobial and other medical therapy has been best described for abdominal infections in adults and has been associated with reduction in mortality [172], the role of source control for pediatric sepsis has been less well elucidated [173].

The importance of source control in children has been shown for skin and deep tissue abscesses and necrotizing fasciitis [173-175]. Despite the relative paucity of pediatric data, source control is an important facet of treatment of sepsis, and should not be delayed. Larger collections containing infected material often are poorly penetrated by IV antimicrobials and contribute to direct and hematogenous spread, ongoing inflammation, and organ dysfunction.

A common, but potentially preventable, source of infection is central line-associated bloodstream infections. Delayed removal of a CVC in neonates and in patients with fungemia or Enterobacteriaceae bacteremia increases the risk of death or slows recovery [176179]. Removal of a CVC that is the source of infection is therefore generally warranted unless extenuating circumstances exist. Fungal infection dictates immediate removal, while in case of coagulase-negative Staphylococcus species or clinically stable patients with infection caused by gram-negative bacilli, infections can often be initially treated through the $\mathrm{CVC}$ as a temporizing measure. The decision to remove the CVC, or not, should ultimately be made based on the pathogen suspected/ recovered and host factors, such as immune status (Supplemental Table 3, Supplemental Digital Content 1, http://links.lww.com/PCC/B139).

\section{Fluid therapy}

17. In healthcare systems with availability of intensive care, we suggest administering up to $40-60 \mathrm{~mL} / \mathrm{kg}$ in bolus fluid (10-20 mL/kg per bolus) over the first hour, titrated to clinical markers of cardiac output and discontinued if signs of fluid overload develop, for the initial resuscitation of children with septic shock or other sepsis-associated organ dysfunction (weak recommendation, low quality of evidence).

18. In healthcare systems with no availability of intensive care and in the absence of hypotension, we recommend against bolus fluid administration while starting main- 
tenance fluids (strong recommendation, high quality of evidence).

19. In healthcare systems with no availability of intensive care, if hypotension is present, we suggest administering up to $40 \mathrm{~mL} / \mathrm{kg}$ in bolus fluid (10-20 $\mathrm{mL} / \mathrm{kg}$ per bolus) over the first hour with titration to clinical markers of cardiac output and discontinued if signs of fluid overload develop (weak recommendation, low quality of evidence).

Remarks: Clinical markers of cardiac output may include heart rate, blood pressure, capillary refill time, level of consciousness, and urine output. In all settings, the need for fluid administration should be guided by frequent reassessment of clinical markers of cardiac output, serial blood lactate measurement and advanced monitoring, when available. Signs of fluid overload that should limit further fluid bolus therapy may include clinical signs of pulmonary edema or new or worsening hepatomegaly.

Rationale: Effective fluid resuscitation in septic shock can correct hypovolemia caused by capillary leak, vasodilation, and fluid losses. Without maintenance of adequate atrial filling pressures, cardiac output will fall and organ perfusion will be compromised.

Three RCTs of different volume resuscitation strategies in children with septic shock in settings in which advanced supportive care (e.g., intubation, mechanical ventilation, and intensive care) was accessible have been published [180-182]. These studies have a combined total of only 316 children and showed no difference in mortality between the restrictive and liberal fluid resuscitation groups (Supplemental Table 4 and Supplemental Fig. 2, Supplemental Digital Content 1, http://links.lww. com/PCC/B139).

In geographic settings in which advanced supportive care, including mechanical ventilation, is limited and/ or intensive care is not routinely accessible, the only large-scale RCT of different bolus fluid volume resuscitation strategies in severe infection in children was the Fluid Expansion as Supportive Therapy (FEAST) study (Supplemental Table 4 and Supplemental Fig. 2, Supplemental Digital Content 1, http://links.lww.com/PCC/ B139) [183]. The FEAST study was conducted in Africa in a low-resource setting without access to PICU admission. Children between 60 days and 12 years old with a severe febrile illness and abnormal perfusion were randomized to either rapid volume expansion with $20 \mathrm{~mL} / \mathrm{kg}$ of IV $0.9 \%$ saline or $5 \%$ albumin or no bolus with maintenance fluid only (control group). Among the 3141 study participants, malaria and anemia were highly prevalent. Overall, the RCT demonstrated a lower mortality after $48 \mathrm{~h}$ in children receiving conservative fluid therapy (i.e., no bolus fluid, maintenance fluid only) than among those given liberal initial fluid therapy (i.e., $20 \mathrm{~mL} / \mathrm{kg}$ fluid bolus with maintenance fluid) with a RR of 0.72 (95\% CI, 0.57-0.9). Notably, 29 additional children enrolled with severe hypotension (systolic blood pressure of $<50 \mathrm{~mm}$ $\mathrm{Hg}$ in children younger than 12 months old, $<60 \mathrm{~mm} \mathrm{Hg}$ in children 1 to 5 years old, and $<70 \mathrm{~mm} \mathrm{Hg}$ in children older than 5 years old) were treated with $40 \mathrm{~mL} / \mathrm{kg}$ fluid bolus per the planned protocol without randomization to the control group. One additional child who was randomized to the control group also received a $40 \mathrm{~mL} / \mathrm{kg}$ fluid bolus due to severe hypotension.

For children with septic shock diagnosed by abnormal perfusion or hypotension in healthcare systems with availability of advanced supportive and intensive care, and in the absence of signs of fluid overload, the panel suggests administering up to $40-60 \mathrm{~mL} / \mathrm{kg}$ fluid bolus therapy in the first hour of resuscitation. Fluid resuscitation should be titrated to clinical markers of cardiac output and discontinued if signs of fluid overload develop. Clinical markers of cardiac output can include heart rate, capillary refill, and urine output. Although no high-quality RCTs demonstrate clear superiority of this practice, numerous observational studies have reported improved patient outcomes with routine administration of up to $40-60 \mathrm{~mL} / \mathrm{kg}$ fluid bolus therapy in the first hour of resuscitation [8, 32, 33, 36, 184-187]. The panel provides only a weak recommendation for this resuscitation strategy in healthcare systems with availability of intensive care because a more restrictive fluid resuscitation strategy has not been shown to be inferior in this setting and indirect data [183] indicate harm from rapid fluid boluses in other settings. For this recommendation, the panel judged the balance of observational data supporting initial fluid bolus therapy to outweigh an indirect suggestion of harm because the generalizability of the FEAST trial to healthcare systems with availability of advanced supportive and intensive care is not clear.

For children with septic shock without signs of fluid overload in low-resource settings where advanced supportive and intensive care is not available, the panel recommends against bolus fluid administration, while starting maintenance fluids, in the first hour if hypotension is not present, and suggests administering up to $40 \mathrm{~mL} / \mathrm{kg}$ in bolus fluid (10-20 mL/kg per bolus) over the first hour if hypotension is present. The strong recommendation against bolus fluid if hypotension is not present was based on the FEAST trial, in which rapid bolus fluid in the first hour of resuscitation increased mortality compared with maintenance fluids only.

For the subset of children with septic shock and hypotension, we suggest cautious administration of fluid bolus therapy in low-resource settings because there are 
insufficient data to conclude that fluid resuscitation is not beneficial in children with septic shock and hypotension. In the FEAST study, all children with "severe" hypotension were treated with $40 \mathrm{~mL} / \mathrm{kg}$ of bolus fluid [183], and so it is not known if fluid bolus therapy was beneficial or harmful in this subgroup of children. It should also be noted that children with gastroenteritis were excluded from FEAST, as ongoing fluid losses should be replaced with IV or oral rehydration as indicated. A recent analysis of children with "moderate" hypotension who were randomized to either fluid bolus or maintenance fluid in the FEAST trial was published after completion of our initial systematic review but considered by the panel to be potentially influential [188]. In this analysis, only children with moderate hypotension were included because children with severe hypotension were not allocated to the control (no bolus) arm. Fluid bolus therapy in children with moderate hypotension was not beneficial or harmful compared with maintenance fluid only (RR of death $=1.48 ; 95 \% \mathrm{CI}, 0.61-3.66 ; \mathrm{p}=0.41$ ). Although children who were reclassified as meeting all three World Health Organization (WHO) shock criteria of cold extremities, prolonged capillary refill greater than $3 \mathrm{~s}$, and weak, fast pulse [14] had $48 \%$ mortality in the bolus groups versus $20 \%$ mortality in the control group, this difference was not statistically significant $(\mathrm{p}=0.07)$. These cases were a very small proportion of the total FEAST trial participants (only 72 [2.3\%] had moderate hypotension and 65 [2\%] met the full WHO shock criteria), and no data were provided about differential patient characteristics between these very small post hoc subgroups to assess for potential confounding. Therefore, until further data are available, the panel suggests cautious administration of fluid bolus therapy for the subset of children with septic shock and hypotension in low-resource settings as a weak recommendation based on low quality of evidence.

Although a suggestion of "up to" $40 \mathrm{~mL} / \mathrm{kg}$ was included for hypotensive shock in low-resource settings because this volume was administered to children with severe hypotension in the FEAST study, fluid administration should always be titrated to clinical markers of cardiac output and discontinued if signs of fluid overload develop. For purposes of this weak recommendation, hypotension can be defined as (1) systolic blood pressure of less than $50 \mathrm{~mm} \mathrm{Hg}$ in children younger than 12 months old, less than $60 \mathrm{~mm} \mathrm{Hg}$ in children 1-5 years old, and less than $70 \mathrm{~mm} \mathrm{Hg}$ in children older than 5 years old [183] or (2) by the WHO criteria of cold extremities "with" prolonged capillary refill greater than $3 \mathrm{~s}$ and weak, fast pulse [14]. Although the panel did not review different approaches to fluid bolus therapy in hypotensive children in low-resource settings, WHO recommends $10-20 \mathrm{~mL} / \mathrm{kg}$ of isotonic crystalloid over 30-60 min, followed by an additional $10 \mathrm{~mL} / \mathrm{kg}$ over $30 \mathrm{~min}$ if condition has not improved and signs of fluid overload, cardiac failure, or neurologic deterioration have not developed [14].

Fluid boluses may be administered as 10 or $20 \mathrm{~mL} / \mathrm{kg}$, according to clinician preference. To facilitate rapid IV fluid administration (as well as other IV therapies, such as antimicrobials and vasoactive medications), clinicians should consider alternative methods of vascular access if initial attempts at peripheral vein cannulation are not immediately successful. Intraosseous access is rapid and effective and recommended by Pediatric Advanced Life Support, Advanced Pediatric Life Support, and the International Liaison Committee on Resuscitation. Ultrasound-guided peripheral IV catheter placement, CVCs, and umbilical venous catheter access are alternatives if the skills are immediately available $[189,190]$. In all healthcare systems, repeat boluses should only be administered after reassessment of hemodynamic status if shock has not resolved and signs of fluid overload are not present.

Although fluid bolus therapy should be discontinued if signs of fluid overload are present or develop, early recognition of fluid overload by clinical examination is a challenge in children. Identifying fluid overload is especially difficult in young children, in whom crackles (rales) are often absent even in the context of gross pulmonary edema. Worsening respiratory status, particularly increasing respiratory rate, radiographic evidence of pulmonary edema in an intubated patient, or new or expanding hepatomegaly may be the only clues of evolving fluid overload. Bedside ultrasound may also be helpful to assess fluid overload, as there is emerging evidence to suggest that a "full" inferior vena cava with minimal variation across the respiratory cycle demonstrated on ultrasound indicates a fluid-replete circulation [191].

\section{We suggest using crystalloids, rather than albumin, for the initial resuscitation of children with septic shock or other sepsis-associated organ dysfunction (weak rec- ommendation, moderate quality of evidence).}

Remarks: Although there is no difference in outcomes, this recommendation takes into consideration cost and other barriers of administering albumin compared with crystalloids.

Rationale: The FEAST trial investigated 3141 African children with infection and impaired perfusion, who were randomly assigned to resuscitation with $5 \%$ human albumin solution or $0.9 \%$ saline boluses or no boluses at admission to the hospital. Although both the albumin 
and $0.9 \%$ saline arms exhibited higher mortality than the no bolus arm, comparing human albumin solution to $0.9 \%$ saline (RR, 1.02; 95\% CI, 0.8-1.28) showed no difference in mortality [183]. In the absence of any clear benefit of albumin administration in children with sepsis, and in view of the additional costs in comparison to crystalloids, problems of availability, and the potential risk of blood-borne infection, we suggest against the routine use of albumin for initial fluid resuscitation in children with sepsis.

21. We suggest using balanced/buffered crystalloids, rather than $0.9 \%$ saline, for the initial resuscitation of children with septic shock or other sepsis-associated organ dysfunction (weak recommendation, very low quality of evidence).

Rationale: Increasing evidence from observational studies and RCTs in adults suggests that resuscitation with crystalloid fluids containing high chloride concentrations (e.g., $0.9 \%$ saline) is associated with hyperchloremic acidosis, systemic inflammation, acute kidney injury (AKI), coagulopathy, and mortality when compared with resuscitation with more balanced/buffered crystalloids (e.g., lactated Ringer's, PlasmaLyte) [192]. Although no pediatric RCTs compare balanced/ buffered crystalloids to $0.9 \%$ saline, there are two large observational studies in children with sepsis [193, 194]. They included a total of 30,532 children with sepsis, 2100 of whom received only balanced/buffered crystalloids for the first $72 \mathrm{~h}$ of hospital admission, and 28,432 who received $0.9 \%$ saline (Supplemental Table 5, Supplemental Digital Content 1, http://links.lww.com/ PCC/B139). These studies showed that use of balanced/ buffered crystalloids was associated with lower mortality (OR, 0.79; 95\% CI, 0.65-0.95) but not AKI (OR, 0.98; 95\% CI, 0.94-1.02) [193, 194]. Indirect evidence from adult patients, including two large RCTs, also demonstrates benefit with balanced/buffered crystalloids over $0.9 \%$ saline, with adult patients who received larger volumes of fluid and those with sepsis exhibiting the greatest benefit $[192,195]$. Taken together, these data support that the desirable consequences of balanced/ buffered crystalloids probably outweigh the undesirable consequences (including cost), especially in those who require large volume of fluid resuscitation. Therefore, pending further high-quality pediatric data, we suggest that balanced/buffered crystalloids should generally be preferred over $0.9 \%$ saline for resuscitation of children with septic shock or other sepsis-associated organ dysfunction without a specific indication for an alternative fluid type (e.g., $0.9 \%$ saline may be preferred in patients with hyponatremia or concern for increased intracranial pressure).

22. We recommend against using starches in the acute resuscitation of children with septic shock or other sepsis-associated organ dysfunction (strong recommendation, moderate quality of evidence).

Rationale: No studies compare starches with other fluids in children. However, in adults with severe sepsis and septic shock (Supplemental Table 6, Supplemental Digital Content 1, http://links.lww.com/PCC/ B139), two large RCTs showed increased risk of mortality, coagulopathy, and AKI in patients receiving hydroxyethyl starch (HES) $[196,197]$. A meta-analysis further confirmed the risk of harm with HES [198]. In the United States, the Food and Drug Administration has restricted the use of HES [199] and the European Medicines Agency has recommended complete suspension of its use [200]. Therefore, we strongly recommend against the use of HES in children with sepsis.

\section{We suggest against using gelatin in the resuscitation of children with septic shock or other sepsis-associated organ dysfunction (weak recommendation, low quality of evidence).}

Rationale: One RCT of gelatin-derived fluid in pediatric septic shock compared it to $0.9 \%$ saline in 60 patients. The estimates were imprecise, and showed no difference in mortality, days of using vasoactive medications, or AKI between the two groups [201] (Supplemental Table 7, Supplemental Digital Content 1, http://links.lww.com/PCC/ B139). In the absence of any data indicating benefit of gelatin in children, we suggest against its use in pediatric sepsis.

\section{Hemodynamic monitoring}

24. We were unable to issue a recommendation about whether to target mean arterial blood pressure (MAP) at the 5th or 50th percentile for age in children with septic shock and other sepsis-associated organ dysfunction. However, in our practice, we target MAP to between the 5th and 50th percentile or greater than 50 th percentile for age.

Rationale: Although no data from RCTs support specific hemodynamic targets in children, evidence suggests that targeting MAP of approximately $65 \mathrm{~mm} \mathrm{Hg}$ (5th percentile) in adults with septic shock may be beneficial [202]. In the absence of evidence from RCTs, we were unable to reach consensus to recommend a specific 
MAP target for children. However, in our practice, 37\% of panel members reported targeting MAP between the 5th and 50th percentile for age and 45\% reported targeting MAP greater than 50th percentile for age. Many panelists also commented that lower blood pressures are acceptable if other hemodynamic variables (e.g., mental status, perfusion, urine output, lactate) are improving. RCTs to define optimal hemodynamic targets, including MAP, are urgently required to inform practice in pediatric sepsis. In settings where direct measurement of MAP is less reliable, systolic blood pressure provides a reasonable alternative.

A previous recommendation to target perfusion pressure (MAP minus central venous pressure [CVP]) lacks supporting data [203]. Prioritizing CVP measurement is also impractical during early resuscitation (such as in most pediatric emergency departments); CVP also provides an unreliable assessment of left ventricular preload.

25. We suggest not using bedside clinical signs in isolation to categorize septic shock in children as "warm" or "cold" (weak recommendation, very low quality of evidence).

26. We suggest using advanced hemodynamic variables, when available, in addition to bedside clinical variables to guide the resuscitation of children with septic shock or other sepsis-associated organ dysfunction (weak recommendation, low quality of evidence).

Remarks: Advanced hemodynamic monitoring may include cardiac output/cardiac index, systemic vascular resistance, or central venous oxygen saturation (Scvo2).

Rationale: The ACCM previously recommended clinical assessment of children in septic shock to differentiate "warm" versus "cold" shock based on extremity temperature, capillary refill, pulse strength, diastolic blood pressure, and pulse pressure. Depending on "warm" or "cold" classification, different resuscitation strategies were suggested (e.g., fluid and vasopressors for "warm" shock and inotropes for "cold" shock). However, a number of observational studies have demonstrated very poor correlation of clinical assessments with cardiac index and systemic vascular resistance as measured by advanced monitoring [204-209]. Indeed, many children who appeared to have "warm" shock by clinical examination had evidence of myocardial dysfunction, thus demonstrating the challenge of using clinical signs alone to direct therapy. Hence, we suggest not attempting to make this distinction using clinical assessments alone, although this categorical distinction may be helpful if advanced hemodynamic monitoring is available to assess patient physiology more accurately. Examples of advanced monitoring include invasive arterial blood pressure monitoring with pulse contour analysis, ultrasound Doppler of the ascending or descending thoracic aorta (suprasternal or esophageal Doppler), cardiac ultrasound/echocardiography [210], or measurement of Scvo2 [190]. All of these variables (other than Scvo2) will provide additional assessment of cardiac index and/or systemic vascular resistance index beyond clinical signs, which may then be used to direct and titrate treatment. There is also emerging evidence that fluid responsiveness may be predicted by aortic blood flow peak velocity variation in mechanically ventilated children [211]. In an RCT of 90 children admitted to a PICU in Egypt, addition of serial echocardiography provided early recognition of septic myocardial dysfunction and hypovolemia that was not apparent on clinical assessment and resulted in faster shock reversal, less fluid overload, shorter LOS, and lower mortality compared with the group without serial echocardiography [210]. When advanced hemodynamic monitoring is available, it is appropriate to target the normal range for variables such as cardiac index, systemic vascular resistance index, stroke index, and Scvo2 (Table 5). No evidence supports targeting a supranormal range of cardiac index.

Until recently, adult guidelines have recommended early goal-directed therapy (EGDT) based on the protocol published by Rivers et al. [212]. This recommendation described the use of a series of "goals" that included CVP and Scvo2. This approach is no longer recommended following a failure to show reduction in mortality in three subsequent large multicenter RCTs [213-215]. In children, there has only been one small RCT supporting the use of a protocolized approach including targeting Scvo2 greater than $70 \%$. This study included 102 children with fluid-refractory septic shock (RSS) and showed a reduced risk of death (RR, 0.3 ; 95\% CI, 0.13-0.68) from a very high baseline mortality of 39\% [26]. No high-quality RCTs have investigated other hemodynamic variables to guide therapy in children (Supplemental Table 8 and Supplemental Fig. 3, Supplemental Digital Content 1, http:// links.lww.com/PCC/B139).

\section{We suggest using trends in blood lactate levels, in addi- tion to clinical assessment, to guide resuscitation of children with septic shock and other sepsis-associated organ dysfunction (weak recommendation, very low quality of evidence).}

Remarks: In children with an elevated blood lactate, repeat testing that reveals a persistent elevation in blood lactate may indicate incomplete hemodynamic resuscitation and should prompt efforts, as needed, to further promote hemodynamic stability. 
Table 5 Normal ranges for advanced monitoring

\begin{tabular}{llll} 
Variable & Formula & Normal range & Units \\
$\mathrm{Cl}$ & $\mathrm{Cl}=$ cardiac output/body surface area & $\mathrm{L} / \mathrm{min} / \mathrm{m}^{2}$ \\
$\mathrm{SI}$ & $\mathrm{SI}=\mathrm{Cl} /$ heart rate & $30-5.5$ & $\mathrm{~mL} / \mathrm{m}^{2}$ \\
$\mathrm{SVRI}$ & $\mathrm{SVRI}=80 \times($ mean arterial pressure-central venous pres- \\
& sure $/ \mathrm{Cl}$ & $800-1600$ & $\mathrm{dyne-s} / \mathrm{cm}^{5} / \mathrm{m}^{2}$ \\
& & \\
\hline
\end{tabular}

$\mathrm{Cl}$ cardiac index, SI stroke index, SVRI systemic vascular resistance index

Rationale: Although blood lactate is not a direct measure of tissue perfusion, increased lactate is associated with worse outcomes in children [11]. Only one pediatric observational study of lactate-guided resuscitation, which included 77 children with sepsis in the ED, was available (Supplemental Table 9, Supplemental Digital Content 1, http://links.lww.com/PCC/B139). This study showed that lactate normalization was associated with a decreased risk of persistent organ dysfunction (RR, 0.46; 95\% CI, 0.29-0.73 and adjusted RR, 0.47; 95\% CI, 0.290.78) [56]. There is also indirect evidence from adult sepsis, with six RCTs (total of 1007 patients) evaluating lactate-guided resuscitation of patients with septic shock [216-221]. The pooled estimates across all RCTs showed significant reduction in mortality compared with resuscitation without lactate monitoring (RR, 0.66; 95\% CI, 0.55-0.81) (Supplemental Table 9, Supplemental Digital Content 1, http://links.lww.com/PCC/B139). Therefore, although there was not sufficient evidence to propose a recommendation to measure lactate to differentiate lowversus high-risk of sepsis among children with infection or suspected infection (see Recommendation 2), we do suggest that blood lactate levels be used to help guide resuscitation of children with established septic shock or other sepsis-associated organ dysfunction.

\section{Vasoactive medications}

28. We suggest using epinephrine, rather than dopamine, in children with septic shock (weak recommendation, low quality of evidence).

29. We suggest using norepinephrine, rather than dopamine, in children with septic shock (weak recommendation, very low quality of evidence).

30. We were unable to issue a recommendation for a specific first-line vasoactive infusion for children with septic shock. However, in our practice, we select either epinephrine or norepinephrine as the first-line vasoactive infusion guided by clinician preference, individual patient physiology, and local system factors.

31. We were unable to issue a recommendation about initiating vasoactive agents through peripheral access in children with septic shock. However, in our practice, we often or sometimes administer a dilute concentra- tion of the initial vasoactive medication through a peripheral vein if central venous access is not readily accessible.

Remarks: It is reasonable to begin vasoactive infusions after $40-60 \mathrm{~mL} / \mathrm{kg}$ of fluid resuscitation if the patient continues to have evidence of abnormal perfusion, or sooner if fluid overload develops or other concerns for fluid administration are present. Either epinephrine or norepinephrine may be administered through a peripheral vein (or intraosseous, if in place) if central venous access is not readily accessible. Dopamine may be substituted as the first-line vasoactive infusion, administered either peripherally or centrally, if epinephrine or norepinephrine is not readily available.

Rationale: Epinephrine and norepinephrine both have vasopressor and inotropic effects, are widely used, and are effective in treating children with fluid-RSS. No studies directly compare epinephrine with norepinephrine. However, epinephrine has been compared with dopamine in two RCTs in children with fluid-RSS [222, 223]. Across both studies, epinephrine was associated with a lower risk of mortality (RR, 0.63; 95\% CI, 0.40-0.99) and more organ failure-free days among survivors by day 28 (MD 4 more days; 95\% CI, 2.0-6.0) (Supplemental Table 10 and Supplemental Fig. 4, Supplemental Digital Content 1, http://links.lww.com/PCC/B139).

Norepinephrine has not been studied in children with septic shock, but in a randomized trial of norepinephrine versus saline in sedated, mechanically ventilated children, mortality was not different between groups (RR, 0.50; 95\% CI, 0.10-2.43; Supplemental Table 11a, Supplemental Digital Content 1, http://links.lww.com/PCC/B139) but the norepinephrine group showed higher urine output $(\mathrm{p}=0.016)$ and improved blood pressure $(\mathrm{p}=0.04)$ suggesting improved perfusion relative to saline [224]. Evidence from adult trials (Supplemental Table 11b, Supplemental Digital Content 1, http://links.lww.com/PCC/ B139) shows a lower mortality rate (RR, 0.93; 95\% CI, 0.86-1.00) and lower incidence of arrhythmias (RR, 0.48; 95\% CI, 0.40-0.58) with norepinephrine than with dopamine, and no difference in mortality with epinephrine 
than with norepinephrine (RR, 0.96; 95\% CI, 0.77-1.21) [225].

Evidence is insufficient to recommend either epinephrine or norepinephrine as the initial vasoactive agent for children with fluid-RSS. In a survey of our panel members, an equal number used epinephrine and norepinephrine as the first-line vasoactive medication with a general preference for epinephrine to treat myocardial dysfunction and low cardiac output and for norepinephrine to increase systemic vascular resistance. It therefore seems reasonable to use either epinephrine or norepinephrine as the initial vasoactive agent, with the choice made based on individual patient physiology, clinician preference, and local system factors. Once cardiac ultrasound/ echocardiography or other advanced monitoring is available, selection of vasoactive therapy should be driven by individual patient physiology.

No pediatric data identify when shock becomes "fluidrefractory" and, thus, to guide when to start vasoactive infusions. However, excessive fluid resuscitation can lead to fluid overload, which has been associated with increased mortality in critically ill children [226]. A trial comparing a fluid-sparing strategy with early initiation of vasoactive medications compared to a fluid-liberal resuscitation strategy is currently ongoing (SQUEEZE trial, ClinicalTrials.gov NCT03080038). Until further data are available, we consider it reasonable to begin vasoactive infusions after $40-60 \mathrm{~mL} / \mathrm{kg}$ of fluid resuscitation if the patient continues to have evidence of abnormal perfusion. Additional fluid resuscitation may be concurrently administered if the patient demonstrates physiologic improvement following each fluid bolus and without signs of fluid overload.

All vasoactive agents, including norepinephrine, may be initiated through peripheral venous (or intraosseous, if in place) access if central venous access is not readily available to avoid delays in therapy [227, 228]. However, central venous access should be obtained as soon as reasonably practicable. In our practice, $82 \%$ of panel members reported at least sometimes administering the initial vasoactive infusion peripherally if central venous or intraosseous access was not readily available, particularly in the emergency department or other nonPICU settings. Most panelists preferred epinephrine or dopamine to norepinephrine if peripheral infusion was needed. Although epinephrine or norepinephrine is the preferred first-line medication, dopamine may be substituted as the first-line vasoactive infusion, administered either peripherally or centrally, if neither epinephrine nor norepinephrine is readily available.

32. We suggest either adding vasopressin or further titrating catecholamines in children with septic shock who require high-dose catecholamines (weak recommendation, low quality of evidence).

Remarks: No consensus was achieved on the optimal threshold for initiating vasopressin. Therefore, this decision should be made according to individual clinician preference.

Rationale: Vasopressin-receptor agonists (vasopressin or terlipressin) have been studied in three RCTs in children (Supplemental Table 12, Supplemental Digital Content 1, http://links.lww.com/PCC/B139). Vasopressin was compared with saline in one study in children with vasodilatory shock [229] and in one study of children with severe lung disease [230]. Terlipressin was compared with usual care in children with septic shock [231]. The mortality rate (RR, 1.14; 95\% CI, 0.80-1.62) and ischemic events (RR, 1.56; 95\% CI, 0.41-5.91) were higher vasopressin/terlipressin. There were fewer vasoactive-free days with vasopressin (median 25.2 days in vasopressin [interquartile range (IQR) 0.0-28.3], median 27.5 days in control [IQR, 23.1-28.9]). In six RCTs in adults, renal replacement therapy was required less often with vasopressin (RR, 0.74; 95\% CI, 0.51-1.08) [232]. Weighing the benefit of avoiding renal replacement therapy against the potential harm from ischemic events and the nonsignificant difference in mortality, we suggest that vasopressin may be added or catecholamines may be further titrated in children on high doses of catecholamines.

33. We were unable to issue a recommendation about adding an inodilator in children with septic shock and cardiac dysfunction despite other vasoactive agents. However, in our practice, we sometimes use inodilators in children with septic shock and evidence of persistent hypoperfusion and cardiac dysfunction despite other vasoactive agents.

Rationale: There are no RCTs of inodilators (including milrinone, dobutamine, or levosimendan) in children with septic shock with persistent hypoperfusion and cardiac dysfunction. A report of two children described improvement in cardiac output with addition of inodilators [233]. A case series of 10 children with meningococcal septic shock treated with milrinone described improved core-to-peripheral temperature gradient, with stable blood pressure and no change in acidosis [234]. These data were not sufficient to formulate a recommendation. However, in our practice, $77 \%$ of panel members reported at least sometimes using inodilators in children with septic shock who had evidence of persistent hypoperfusion and cardiac dysfunction despite other vasoactive 
agents, typically in a PICU with advanced hemodynamic monitoring available.

\section{Ventilation}

34. We were unable to issue a recommendation about whether to intubate children with fluid-refractory, catecholamine-resistant septic shock. However, in our practice, we commonly intubate children with fluidrefractory, catecholamine-resistant septic shock without respiratory failure.

Rationale: There are no RCTs and/or observational studies of children receiving early intubation for refractory shock without respiratory failure compared with delayed or no intubation for the same condition, nor is there suitable indirect evidence to substantiate a formal recommendation. However, it is well understood that a high metabolic demand from refractory shock typically indicated by progressive lactic acidemia and end-organ dysfunction can be, at least in part, mitigated by early invasive mechanical ventilation even without clinical symptoms of acute pulmonary edema or respiratory failure [235-237]. Furthermore, chest radiograph findings can "lag" behind clinical deterioration [238, 239] such that patients with refractory shock and a "negative" chest radiograph may still progress toward more overt ARDS. Lung ultrasound may provide an alternative tool to chest radiograph in detecting lung pathology, but its utility to identify which sepsis patients may benefit from early mechanical ventilation is not yet clear [240-243]. For these reasons, 48\% of panel members often or always and 35\% sometimes intubate children with fluid-refractory, catecholamine-resistant septic shock even in the absence of clear respiratory failure, while $17 \%$ rarely or never do so. Of note, when intubating, caution should be exercised to avoid worsening hypotension or precipitating cardiac arrest as medications used for inducing anesthesia at the time of tracheal intubation, along with conversion from spontaneous breathing to use of positive pressure ventilation, may result in a transient deterioration in patient hemodynamics. The panel does recognize that in some settings, invasive mechanical ventilation may not be available or feasible-or may even be detrimental. In these instances, transport of the patient to a higher level of care can be life-saving.

35. We suggest not to use etomidate when intubating children with septic shock or other sepsis-associated organ dysfunction (weak recommendation, low quality of evidence).

Rationale: Etomidate is a short-acting IV anesthetic agent that has been used for inducing anesthesia and sedation for tracheal intubation in patients with unstable hemodynamics. However, concerns regarding the drug's effect on adrenal function have been raised in adult studies. No RCTs exist in critically ill children with or without sepsis comparing etomidate to another anesthesia/sedative regimen. Two observational studies included children. One study from 1984 [244] enrolled acutely injured adults and children (44 intubated with etomidate vs 90 intubated with a benzodiazepine and opioid). A more recent study [245] enrolled children with meningococcal sepsis or septic shock with 23 intubated with etomidate as compared with 37 intubated with any other combination of sedatives. Although caution must be taken given the small sample size, each of these studies reported higher mortality after use of etomidate (pooled OR, 4.51; 95\% CI, 1.82-11.16) (Supplemental Table 13, Supplemental Digital Content 1, http://links.lww.com/PCC/B139). In addition, den Brinker et al. [245] reported a significant association of etomidate with adrenal insufficiency, with cortisol to adrenocorticotropin hormone $(\mathrm{ACTH})$ ratios decreasing by $83 \%$ after etomidate exposure. Indirect evidence is available from four RCTs in adults [246-249]. In the largest of these trials, Jabre et al. [249] compared 234 critically ill adults intubated with etomidate to 235 intubated with an alternative medication regimen and found higher adrenal insufficiency in the etomidate group (OR, 1.79; 95\% CI, 1.37-2.36). Pooled odds of all four adult studies was 1.89 (95\% CI, 1.47-2.44) with all studies suggesting significantly increased risk of adrenal insufficiency after etomidate administration. Importantly, this effect was seen even after one dose of etomidate. Unfortunately, there is no conclusive evidence to recommend an optimal alternative induction agent to etomidate, although ketamine and fentanyl are routinely available and can offer favorable hemodynamic profiles in the setting of shock.

\section{We suggest a trial of noninvasive mechanical ventila- tion (over invasive mechanical ventilation) in children with sepsis-induced pediatric ARDS (PARDS) without a clear indication for intubation and who are respond- ing to initial resuscitation (weak recommendation, very low quality of evidence).}

Remarks: When noninvasive mechanical ventilation is initiated, clinicians should carefully and frequently reevaluate the patient's condition.

Rationale: Noninvasive mechanical ventilation with continuous positive airway pressure ventilation or bilevel positive airway pressure ventilation may allow for decreased work of breathing and improved oxygenation in the face of sepsis-induced PARDS. Therefore, 
it is possible to avoid intubation in sepsis patients who are identified early with mild PARDS physiology and no evidence of advancing end-organ dysfunction. However, no RCTs in either critically ill children or children with sepsis-induced PARDS compare the effect of noninvasive ventilation to invasive mechanical ventilation on clinical outcomes. Observational studies have tested whether noninvasive mechanical ventilation could mitigate the need for invasive mechanical ventilation but none specifically focused on children with sepsis [250-256]. We undertook a meta-analysis of three observational studies that evaluated the association of noninvasive mechanical ventilation with mortality in a general PICU population $[252,254,257]$. Using unadjusted estimates pooled from the data across all three studies, we found noninvasive ventilation to be associated with a decreased risk of death (RR, 0.21; 95\% CI, 0.09-0.47) (Supplemental Fig. 5, Supplemental Digital Content 1, http://links.lww.com/PCC/ B139). One additional RCT in immunocompromised children with acute respiratory dysfunction did not find that early noninvasive ventilation reduced intubation compared with standard care, but the trial was small (42 participants) due to low consent and overall slow recruitment and the direct relevance to children with sepsisinduced PARDS without a clear indication for intubation and who are responding to initial resuscitation was not clear [258]. Thus, it is reasonable to try noninvasive mechanical ventilation in children with sepsis-induced PARDS who do not have a clear indication for intubation. However, noninvasive ventilation should be reserved for children with sepsis who are responding to initial resuscitation, do not have evidence for ongoing or worsening end-organ dysfunction, and in whom close monitoring and frequent reevaluation can be ensured [253, 255, 259]. This recommendation for children with sepsis-induced PARDS aligns with the 2015 PALICC [260] and 2017 Pediatric Mechanical Ventilation Consensus Conference (PEMVECC) [261] guidelines.

37. We suggest using high positive end-expiratory pressure (PEEP) in children with sepsis-induced PARDS (weak recommendation, very low quality of evidence).

Remarks: The exact level of high PEEP has not been tested or determined in PARDS patients. Some RCTs and observational studies in PARDS have used and advocated for use of the ARDS-network PEEP to Fio2 grid though adverse hemodynamic effects of high PEEP may be more prominent in children with septic shock.

Rationale: PEEP helps to prevent alveolar collapse, restore end-expiratory lung volume, and improve mean airway pressures, all of which help to improve adequate oxygenation in PARDS patients and minimize unnecessary use of high Fio2. Adult ARDS patients have been successfully managed with judicious and strict application of a PEEP/Fio2 grid, initially implemented in an ARDS-network trial [262]. This grid has been applied in children with PARDS enrolled in RCTs [263], but a pediatric-specific PEEP/Fio2 grid has not been determined or validated. In 2017, a multicenter observational study by the Collaborative Pediatric Critical Care Research Network reported that pediatric critical care clinicians almost uniformly limit PEEP to $10 \mathrm{~cm}$ $\mathrm{H} 2 \mathrm{O}$ irrespective of oxygenation and Fio2 [264]. This is in contrast to the PEMVECC [261] and PALICC [24] recommendations for use of PEEP in excess of $15 \mathrm{~cm}$ $\mathrm{H} 2 \mathrm{O}$ for severe PARDS patients. Our panel reviewed several observational studies of PARDS patients, all published since 2007, each including 12-30\% sepsis-induced PARDS [264-276]. The largest, a multicenter study by Khemani et al. [276], evaluated 1134 PARDS patients of whom $26 \%$ were managed with lower PEEP relative to ARDS Network (ARDSnet) protocol and experienced greater mortality than those managed in accordance with a higher PEEP strategy as recommended by the ARDSnet PEEP/Fio2 grid (Supplemental Table 14, Supplemental Digital Content 1, http://links.lww.com/PCC/ B139). After adjustment for relevant comorbidities, pediatric patients managed with a PEEP strategy at or above that recommended by the ARDSnet low PEEP/Fio2 grid had a decreased odds of death compared with children managed with PEEP lower than that recommended by the ARDSnet low PEEP/Fio2 grid (adjusted OR, 0.50; 95\% CI, 0.31-0.81).

The panel concluded that PEEP levels greater than $10 \mathrm{~cm} \mathrm{H2O}$ may be necessary with progressive hypoxemia, with the precise amount of "high" PEEP carefully titrated for each individual while attending to the potential adverse hemodynamic effects of increasing intrathoracic pressure in children with septic shock. Therefore, although the optimal approach to setting PEEP has not yet been determined in children with PARDS, carefully increasing PEEP for children with sepsis-induced PARDS who require Fio 2 exceeding $60 \%$ and/or exhibit ongoing hypoxemia is reasonable, rather than continuing to manage such children with a low-PEEP or moderate-PEEP strategy of less than or equal to $10 \mathrm{~cm} \mathrm{H} 2 \mathrm{O}$.

\section{We cannot suggest for or against the use of recruitment maneuvers in children with sepsis-induced PARDS and refractory hypoxemia.}

Remarks: If a recruitment maneuver is considered, the use of a stepwise, incremental and decremental PEEP titration maneuver is preferred over sustained inflation techniques that have not been optimized through direct 
testing in PARDS patients. All PARDS patients must be carefully monitored for tolerance of the maneuver.

Rationale: ARDS is characterized by decreased lung compliance, risk for atelectasis, and increased intrapulmonary shunt. Recruitment maneuvers have been used in both children and adults temporarily to increase transpulmonary pressure to recruit lung units with the goal of improving both oxygenation and ventilation. Most recruitment maneuvers include either sustained inflation or a step-wise incremental or decremental PEEP titration methodology. However, many clinicians and researchers remain concerned that the optimal strategy for lung recruitment has not been determined and injudicious implementation of recruitment maneuvers can result in hemodynamic compromise [277], hypercarbia [278], and/or ventilator-induced lung injury [279]. PEMVECC did not recommend use of recruitment maneuvers in children, citing an overall lack of evidence in this area [261]. In contrast, the 2015 PALICC provided a weak recommendation in favor of recruitment maneuvers with prioritization of a slow stepwise incremental and decremental PEEP method [24].

Two observational studies are potentially informative about use of recruitment maneuvers in children with sepsis-induced PARDS [267, 268]. (Supplemental Table 15, Supplemental Digital Content 1, http://links.lww.com/ PCC/B139) First, Boriosi et al. [280] enrolled 21 children with lung injury, of whom $66 \%$ had sepsis, and used incremental PEEP recruitment maneuvers. Patients experienced improved oxygenation as measured by both the Pao2 to Fio2 (P/F) ratio and alveolar-to-arterial oxygen gradient for the $4 \mathrm{~h}$ after recruitment. Second, Duff et al. [281] enrolled 32 children and used the sustained inflation technique, which also resulted in improved oxygenation for the ensuing $6 \mathrm{~h}$. However, neither study tested the association of recruitment maneuvers with clinical outcomes, such as ventilator days or mortality. Consequently, despite the potential for benefit for some patients coupled with the possibility of harm [282, 283], insufficient data do not allow us to recommend either for or against recruitment maneuvers in sepsis-induced PARDS patients at this time.

39. We suggest a trial of prone positioning in children with sepsis and severe PARDS (weak recommendation, low quality of evidence).

Remarks: Research trials in adults with ARDS and children with PARDS have emphasized prone positioning for at least $12 \mathrm{~h}$ per day, as tolerated.
Rationale: Prone positioning almost uniformly improves oxygenation in adults with ARDS and children with PARDS. Although the exact mechanisms continue to be elucidated, prone position has been shown to recruit areas of collapsed, de-recruited lung with resultant improved elastance, decreased lung stress and strain, and improved functional residual capacity [284]. Given that pulmonary perfusion is thought to be consistent both dorsally and ventrally, an improvement in lung aeration can be met with continued perfusion, thereby reducing ventilation-perfusion mismatching [285]. Most recent RCTs in adults support use of prone positioning as a potentially life-saving management strategy (Supplemental Table 16, Supplemental Digital Content 1, http://links.lww.com/ PCC/B139), especially in those meeting severe ARDS criteria (i.e., $\mathrm{P} / \mathrm{F}<150 \mathrm{~mm} \mathrm{Hg}$ ) [286]. This benefit is seen particularly in patients who are positioned for prolonged periods of time, most commonly reported as $12-20 \mathrm{~h}$ per day. Two pediatric RCTs tested the use of prone positioning in PARDS patients [263,287]. Pooled analyses of these two studies yielded a RR of 0.99 (95\% CI, 0.36-2.69) for mortality in prone positioning as compared with supine positioning for this patient population (Supplemental Table 16 and Supplemental Fig. 6, Supplemental Digital Content 1, http://links.lww.com/PCC/B139). Importantly, no serious adverse events were reported in these trials, although the prone positioning methodology was protocolized in each with particular attention to avoid accidental endotracheal extubation and pressure injury. PALICC [24] did not recommend routine use of prone positioning in PARDS patients but suggested its consideration in severe PARDS. The panel noted that the National Institutes of Health has approved and funded an international RCT of prone positioning in severe PARDS (ClinicalTrials.gov identifier NCT02902055).

40. We recommend against the routine use of inhaled nitric oxide (iNO) in all children with sepsis-induced PARDS (strong recommendation, low quality of evidence).

41. We suggest using iNO as a rescue therapy in children with sepsis-induced PARDS and refractory hypoxemia after other oxygenation strategies have been optimized (weak recommendation, moderate quality of evidence).

Rationale: The presumptive mechanism of sepsisinduced PARDS involves alveolar epithelial injury, vascular endothelial injury, and activation of inflammatory, fibrosis, and coagulation cascades. As such, PARDS is not a disease process primarily of pulmonary arterial 
hypertension, the therapeutic target of iNO therapy, and so is not recommended for routine use in children with sepsis-associated PARDS. Nonetheless, many PARDS patients have comorbidities that include risk for pulmonary hypertension (e.g., chronic lung disease after prematurity, congenital heart disease after repair or palliation) or clinical features, such as acidemia and hypoxemia that increase pulmonary arterial pressures. Thus, iNO therapy may be considered in children with documented pulmonary hypertension or severe right ventricular dysfunction $[239,288]$. Such use of iNO in sepsis must be balanced against its lack of availability or high cost in many areas of the world and, that once in place, iNO use carries a potential patient safety consideration as inadvertent and abrupt discontinuation of the therapy can result in a rapid and potentially life-threatening rebound pulmonary hypertensive crisis.

Several small RCTs [289-291] and observational studies have described significant improvement in oxygenation after iNO therapy [292]. Many, but not all, of these studies include patients with sepsis [290, 291, 293-296], and few analyze longer term, clinically relevant outcomes such as mortality. A 2016 Cochrane review indicated no mortality benefit from iNO administration (RR, 0.78; 95\% CI, 0.51-1.18) in three RCTs [297]. Our analysis of two recent observational studies, one conducted in children on ECMO and another in children with severe PARDS, respectively, suggest possible increased mortality risk [294, 296], whereas one RCT of 55 PARDS patients indicated improved duration of mechanical ventilation in PARDS survivors [291] (Supplemental Table 17 and Supplemental Fig. 7, Supplemental Digital Content 1, http://links.lww.com/ PCC/B139). Taken together, these data do not support "routine" use of iNO in all children with sepsisinduced PARDS but do raise the potential for benefit as an emergency rescue therapy for severe, sepsis-induced PARDS with refractory hypoxemia after other oxygenation strategies have been optimized. Emergency rescue use of iNO may allow time to realize benefit from other therapies, such as lung recruitment, or provide a bridge to ECMO or another intervention. However, when iNO is used, we agree with the PALICC recommendation that "assessment of benefit must be undertaken promptly and serially to minimize toxicity and to eliminate continued use without established effect" [24]. These recommendations align with the 2004 guidelines for use of iNO therapy in neonates and children issued by the European Society for Pediatric and Neonatal Intensive Care [288], PALICC guidelines [24], and a
2017 Cochrane review [292] as no relevant change in evidence has become available.

42. We were unable to issue a recommendation to use high-frequency oscillatory ventilation (HFOV) versus conventional ventilation in children with sepsisinduced PARDS. However, in our practice, there is no preference to use or not use HFOV in patients with severe PARDS and refractory hypoxia.

Rationale: HFOV provides a sustained mean airway pressure with superimposed high frequency, pendellufttype, oscillatory breaths that may improve oxygenation in patients with moderate-to-severe lung disease while minimizing barotrauma, volutrauma, and atelectrauma. However, the most efficacious timing of application, optimal settings, and ideal population of patients likely to benefit have not been well established. HFOV may be difficult to apply effectively in centers with little experience and is not universally available. Despite these practical limitations, both PALICC [24] and PEMVECC [261] endorsed cautionary use of HFOV as an alternative type therapy in patients with severe PARDS. In our panel, clinicians who use versus those who do not use HFOV in patients with severe PARDS and refractory hypoxia were nearly evenly distributed.

Application of HFOV in adult ARDS patients has yielded concerning results due to a potentially increased mortality observed in the adult OSCILLATE RCT [298] and a neutral result in the adult OSCAR RCT [299]. Pediatric data include two observational studies with a nonHFOV control group and three randomized trials. In the two observational studies, oxygenation improved with HFOV relative to conventional ventilation but there was a nonsignificant trend toward increased mortality (Guo et al. [300]: $34.6 \%$ vs $22.7 \%$; adjusted OR, 2.74; $95 \%$ CI, $0.52-14.6$ and Bateman et al. [301]: $25 \%$ vs $17 \%$; adjusted OR, 1.28; 95\% CI, 0.92-1.79). Among three small RCTs, however, a trend toward reduced mortality in those managed with HFOV was observed (pooled RR, 0.77; 95\% CI, 0.43-1.36) [302-304]. A large, multicenter, international RCT of HFOV compared with conventional mechanical ventilation in severe PARDS patients, including children with and without sepsis, is underway and will seek to address many of these issues (www.clinicaltrials.gov/ NCT02902055).

43. We suggest using neuromuscular blockade in children with sepsis and severe PARDS (weak recommendation, very low quality of evidence). 
Remarks: The exact duration of neuromuscular blockade to use in severe PARDS patients has not been determined to date. Most of the adult RCT data and pediatric observational data support treatment for 24-48 h after ARDS onset.

Rationale: Indirect evidence from three adult RCTs [305-307] found that early use of neuromuscular blocking agents (NMBAs) for up to $48 \mathrm{~h}$ in adults with severe ARDS, defined as $\mathrm{P} / \mathrm{F}$ ratio less than $150 \mathrm{~mm} \mathrm{Hg}$, improved 90-day survival and shortened duration of mechanical ventilation without increasing muscle weakness. In a multi-center double-blind RCT [307], 340 patients with early severe ARDS, meeting criteria within $48 \mathrm{~h}$, were randomized to receive either cisatracurium besylate or placebo once adequately sedated. After adjustment for baseline P/F, plateau pressure, and the Simplified Acute Physiology Score, the cisatracurium group had a hazard ratio for death at 90 days of 0.68 (95\% CI, 0.48-0.98) compared with the placebo group. Early use of NMBAs was also associated with decreased organ system dysfunction, less air leak, and a decreased pro-inflammatory response [308]. These findings remained consistent when combined with earlier smaller studies from the same group of investigators in a meta-analysis. However, the more recent ROSE trial of early neuromuscular blockade in adults with moderate to severe ARDS was stopped for futility at the second interim analysis (enrollment of 1006 patients) with a 90-day mortality difference of $42.5 \%$ in the intervention group versus $42.8 \%$ in the control group. In this study, the intervention group received continuous cisatracurium and deep sedation for $48 \mathrm{~h}$ compared with the control group that received lighter sedation targets (Richmond Agitation Scale of 0 to -1 ). Both groups received low tidal volume ventilation with high PEEP strategy. Notably, only $13.8 \%$ of patients enrolled in ROSE had nonpulmonary sepsis as a primary diagnosis.

In pediatrics, there are no prospective data regarding the use of NMBAs in PARDS (with or without sepsis), although there is an ongoing pediatric trial in the Netherlands (ClinicalTrials.gov NCT02902055). In one large retrospective study of 317 children with PARDS, of whom 23\% experienced sepsis-induced PARDS [309], mortality was lower in those children treated with neuromuscular blockade ( $8.8 \%$ vs $17.7 \%$ ). However, duration of mechanical ventilation was longer in the treatment group and proportion with neuromuscular weakness was not assessed (Supplemental Table 18, Supplemental Digital Content 1, http://links.lww.com/PCC/B139).

\section{Corticosteroids}

44. We suggest against using IV hydrocortisone to treat children with septic shock if fluid resuscitation and vasopressor therapy are able to restore hemodynamic stability (weak recommendation, low quality of evidence).

45. We suggest that either IV hydrocortisone or no hydrocortisone may be used if adequate fluid resuscitation and vasopressor therapy are not able to restore hemodynamic stability (weak recommendation, low quality of evidence).

Rationale: A potential role for IV hydrocortisone as adjunctive therapy for septic shock is supported by various roles of cortisol in homeostasis and the stress response. For example, cortisol directly decreases reuptake of norepinephrine [310], augments beta-adrenergic receptor sensitivity in the heart, and enhances calcium availability in myocardial and vascular smooth muscle cells [311] promoting myocardial contractility and vasoconstriction, respectively. Cortisol helps to inhibit prostacyclin and endogenous nitric oxide production, resulting in increased vascular tone [312], modulation of capillary leak [313], and augmentation of the betaadrenergic receptor in the heart [312]. However, potential adverse side effects of corticosteroid therapy include hyperglycemia [314, 315], catabolism-related diffuse neuromuscular weakness (including the diaphragm) [316, 317], and hospital-acquired infections [318]. These effects may be under-appreciated in critically ill patients but can contribute to worse outcomes [319].

At least one pediatric [320] and several adult [321] interventional trials examining adjunctive corticosteroids for septic shock have concluded that this drug class hastens resolution of shock. Of the four adult, high-quality contemporary RCTs, two reported a mortality reduction and two did not [322-326]. A recent meta-analysis of 42 RCTs including 9969 adults and 225 children with sepsis found that corticosteroids possibly result in a small reduction in short-term mortality (RR, 0.93; 95\% CI, 0.84-1.03), long-term mortality (0.94; 95\% CI, 0.891.00 ), faster resolution of shock, and shorter LOS, while also possibly increasing the risk of neuromuscular weakness (RR, 1.21; 95\% CI, 1.01-1.52) [327]. Despite a weak recommendation to treat sepsis with hydrocortisone based on the findings noted in the overall meta-analysis [328], the pediatric studies enrolled a combined small number of subjects, reported inconsistent conclusions, had methodologic limitations, and did not demonstrate an overall mortality reduction [320, 329-331] (Supplemental Table 19, Supplemental Digital Content 1, http:// links.lww.com/PCC/B139).

Observational cohort studies have reported either harm or no benefit with hydrocortisone in children with septic shock [5, 332-336]. For example, a retrospective analysis of the REsearching severe Sepsis and Organ 
dysfunction in children: a gLobal perspectiVE trial of activated protein $\mathrm{C}$ in pediatric sepsis found no differences in mortality, duration of mechanical ventilation and vasoactive-inotropic support, or PICU stay among 193 children who received and 284 who did not receive open-labeled corticosteroids [333]. Despite the post hoc analysis, age, sex, Pediatric Risk of Mortality (PRISM) III scores, baseline number of dysfunctional organs, and baseline Pediatric Overall Performance Category scores did not differ between corticosteroid-treated and corticosteroid nontreated groups.

Several pediatric and adult studies have attempted to use random cortisol and/or cosyntropin-stimulated cortisol serum concentrations to identify which patients with septic shock may benefit from hydrocortisone therapy, but reliable cutoffs have not been clearly identified. Challenges relate to variability in (1) the cortisol assay itself; (2) cortisol metabolism (11-beta-hydroxysteroid dehydrogenase) during sepsis; (3) corticosteroid-binding globulin concentrations; and (4) multiple tissue (e.g., elastase, anti-glucocorticoid compounds) and cellular (e.g., glucocorticoid receptor) factors. Therefore, use of random cortisol or stimulation tests to guide corticosteroid prescription in children with septic shock cannot be recommended as this time. However, for any patient with a clinical concern for primary adrenal insufficiency (e.g., a patient with significant and unexplained hypoglycemia, hyponatremia, and/or hyperkalemia), a highdose cosyntropin-stimulation test should be performed. Interpretation should focus on the baseline serum ACTH concentration (above normal indicating primary adrenal insufficiency) and the 60-minute stimulated serum cortisol concentration $(<18 \mu \mathrm{g} / \mathrm{dL}$ indicating primary adrenal insufficiency) [337].

In summary, no high-quality investigations currently support or refute the routine use of adjunctive corticosteroids for pediatric septic shock or other sepsis-associated organ dysfunction. At the time of this publication, an RCT is in progress to examine the potential risks and benefits of adjunctive hydrocortisone for fluid and vasoactive-inotropic recalcitrant septic shock in children. However, this uncertainty does not apply to children presenting with septic shock or other sepsis-associated organ dysfunction who also have acute or chronic corticosteroid exposure, hypothalamic-pituitary-adrenal axis disorders, congenital adrenal hyperplasia or other corticosteroid-related endocrinopathies, or have recently been treated with ketoconazole or etomidate, for whom prescription of stress-dose hydrocortisone is indicated, with or without evaluation of the adrenal axis [338].

\section{Endocrine and metabolic}

46. We recommend against insulin therapy to maintain a blood glucose target at or below $140 \mathrm{mg} / \mathrm{dL}$ (7.8 mmol/L) (strong recommendation, moderate quality of evidence).

47. We were unable to issue a recommendation regarding what blood glucose range to target for children with septic shock or other sepsis-associated organ dysfunction. However, in our practice, there was consensus to target blood glucose levels below $180 \mathrm{mg} / \mathrm{dL}$ $(10 \mathrm{mmol} / \mathrm{L})$ but there was not consensus about the lower limit of the target range.

Rationale: Although hyperglycemia has been associated with poor outcomes in numerous studies of critically ill children and adults, three prospective multicenter RCTs of glucose control to a low target range (including 50-80, $70-100,72-126,80-110 \mathrm{mg} / \mathrm{dL}$ or $2.8-4.4,3.9-5.6$, 4.0-7.0, 4.4-6.1 mmol/L) have not demonstrated clinical benefit in children [339-341] (Supplemental Table 20, Supplemental Digital Content 1, http://links.lww.com/ PCC/B139). One single-center RCT did show substantial mortality benefit, but there was a high rate of severe hypoglycemia and the higher target range cohort had substantially higher blood glucose levels than those used in the other multicenter RCTs [342]. A trial involving children with burn injuries, a unique PICU population, demonstrated no mortality benefit but did find a significant reduction in morbidity [343]. Notably, all trials included sepsis patients but none targeted them exclusively. Meta-analyses of all published prospective trials in children have shown no clinical benefits overall, but showed a substantially higher risk of hypoglycemia when using insulin therapy to maintain a glucose target below $140 \mathrm{mg} / \mathrm{dL}(7.8 \mathrm{mmol} / \mathrm{L})[344,345]$. Even brief episodes of severe hypoglycemia during septic shock in children may be a risk factor for poor long-term developmental outcomes [346-349].

Treating hyperglycemia greater than or equal to $180 \mathrm{mg} / \mathrm{dL}(\geq 10 \mathrm{mmol} / \mathrm{L})$ may be desirable as incidence of insulin-induced hypoglycemia in the studied pediatric cohorts with targets of $140-180 \mathrm{mg} / \mathrm{dL}$ $(7.8-10.0 \mathrm{mmol} / \mathrm{L})$ is extremely low. There are, however, no direct comparisons between treatment to less than $180 \mathrm{mg} / \mathrm{dL}(10.0 \mathrm{mmol} / \mathrm{L})$ and no treatment. Therefore, evidence cannot definitively guide this therapeutic target. However, given that the guidelines for adults recommend an upper limit of $180 \mathrm{mg} / \mathrm{dL}(10 \mathrm{mmol} / \mathrm{L})$ 
and given the lack of harm demonstrated in the pediatric trials with those targets, treating children with septic shock or other sepsis-associated organ dysfunction with IV insulin with a goal upper blood glucose target of $180 \mathrm{mg} / \mathrm{dL}(10 \mathrm{mmol} / \mathrm{L})$ is reasonable. The lower target, that is, the glucose concentration below which insulin infusion should be discontinued, has also not been specifically studied, but is reasonable to set at $140-150 \mathrm{mg} /$ $\mathrm{dL}(7.8-8.3 \mathrm{mmol} / \mathrm{L})$, based on similar principles. In a survey of our panel members, $32.5 \%$ always or often and $17.5 \%$ sometimes target glucose levels between 140 and $180 \mathrm{mg} / \mathrm{dL}$. Regardless of the glucose target, the overriding goal during insulin therapy should be avoidance of hypoglycemia.

48. We were unable to issue a recommendation as to whether to target normal blood calcium levels in children with septic shock or sepsis-associated organ dysfunction. However, in our practice, we often target normal calcium levels for children with septic shock requiring vasoactive infusion support.

Rationale: Calcium has an essential role in nearly all cellular processes, including myocardial contractility and vasomotor tone. As such, intracellular and circulating levels of calcium are tightly regulated. During septic shock, derangements in calcium regulation frequently occur in critically ill adults and children. However, a systematic review of adult literature found no evidence to support treating hypocalcemia of critical illness [350]. Calcium supplementation may actually worsen organ dysfunction and is correlated with adverse outcomes in critically ill adult patients receiving PN [351]. Although the prevalence of hypocalcemia in critically ill children has been reported to be up to $75 \%$ and is associated with organ dysfunction [352], no studies in children with septic shock have investigated the effect of calcium supplementation to treat hypocalcemia. However, in our practice, $65 \%$ of panel members always or often and $20 \%$ sometimes target normal calcium levels with parenteral calcium administration in children with septic shock requiring vasoactive infusion support. Only $15 \%$ of panel members rarely or never target normal calcium levels.

49. We suggest against the routine use of levothyroxine in children with septic shock and other sepsis-associated organ dysfunction in a sick euthyroid state (weak recommendation, low quality of evidence).

Rationale: Critically ill children, similar to adults, develop low tri-iodothyronine (T3) and low normal thyroxine (T4) concentrations without the compensatory rise in thyroid-stimulating hormone that is typical of the "sick euthyroid" state or hypothyroxinemia of nonthyroidal illness [353]. The decrease in T3 is due both to increased thyroid hormone turnover and to decreased de-iodination of T4 to T3, with redirection of T4 metabolism toward higher levels of biologically inactive reverse T3. The magnitude of the drop in T3 within the first $24 \mathrm{~h}$ of illness reflects the severity of illness [354]. Although of theoretical benefit, few trials of thyroid hormone replacement have been conducted in critically ill children and none in children with sepsis. Two prospective RCTs in children undergoing cardiac surgery (without sepsis) showed no difference in mortality, vasoactive days, or PICU LOS [355, 356]. One open-label study in premature neonates also showed no difference in clinical outcomes [357]. Taken together, there are no direct data to inform a recommendation for children with sepsis, and no indirect data from other critically ill children to support a recommendation for the routine use of levothyroxine in children with septic shock and other sepsis-associated organ dysfunction in a sick euthyroid state.

\section{We suggest either antipyretic therapy or a permissive approach to fever in children with septic shock or other sepsis-associated organ dysfunction (weak recommen- dation, moderate quality of evidence).}

Rationale: Fever is a complex physiologic response associated with sepsis, and it remains unclear whether fever is a beneficial [358] or a harmful [359] response to infection. Potential benefits include inhibiting the growth of some pathogens and increased neutrophil production and lymphocyte proliferation. Conversely, fever is associated with an increased metabolic rate (which may or may not have detrimental effects in patients with sepsis) and may impair some components of immune function. Fever can also make patients uncomfortable [360]. Thus, the putative benefits of maintaining normothermia by treating fever are unclear.

No direct evidence for or against the use of antipyretics in febrile children with sepsis-associated organ dysfunction exists. Rather, the panel had to consider indirect data extrapolated from studies in adults. One systematic review of adult patients studied the use of antipyretics and physical cooling methods included eight RCTs (1507 patients) and eight observational studies (17,432 patients) [361]. This study had 28-day mortality as the primary outcome, with additional outcomes of early mortality (i.e., death on or prior to day 14), frequency of acquisition of hospital-acquired infection, frequency of shock reversal, and mean changes in body temperature, heart rate, and minute ventilation. No difference was noted in 28-day mortality. Effects on early mortality differed 
between the randomized (favored reduced mortality with antipyretic therapy) and observational (favored increased mortality with antipyretic therapy) studies. Although antipyretic therapy successfully decreased body temperature, there was no effect on heart rate, minute ventilation, shock reversal, or acquisition of nosocomial infections. This study did not assess outcome measures of patient comfort. Based on available data, we are not able to recommend the optimal approach to fever in children with sepsis. However, it is reasonable to provide antipyretic therapy to optimize patient comfort, to reduce metabolic demand under certain clinical scenarios (e.g., refractory shock, pulmonary hypertension), and to reduce extreme body temperatures.

\section{Nutrition}

51. We were unable to issue a recommendation regarding early hypocaloric/trophic enteral feeding followed by slow increase to full enteral feeding versus early full enteral feeding in children with septic shock or sepsisassociated organ dysfunction without contraindications to enteral feeding. However, in our practice, there is a preference to commence early enteral nutrition within $48 \mathrm{~h}$ of admission in children with septic shock or sepsis-associated organ dysfunction who have no contraindications to enteral nutrition and to increase enteral nutrition in a stepwise fashion until nutritional goals are met.

Rationale: No studies examine the enteral nutrition advancement strategy in children with septic shock or other sepsis-associated organ dysfunction. Indirect evidence from a small RCT in critically ill children examines early $(6-24 \mathrm{~h})$ versus late enteral nutrition $(>24 \mathrm{~h})$ in, respectively, 57 and 52 children [362]. Early enteral feeding had no effect on duration of PICU stay, but a trend toward lower mortality in the early feeding group ( $30 \%$ in early feeding vs $48 \%$ in late feeding; $\mathrm{p}=0.07$ ) was shown. There is also indirect evidence from the EDEN trial in adults [363] in which 200 patients were randomized to receive either trophic or full enteral feeding for the first 6 days. This study demonstrated no difference in number of ventilator-free days, mortality at 60 days, or infectious complications, but trophic enteral feeding was associated with less gastrointestinal intolerance. Because neither of these studies was conclusive nor directly studied children with septic shock or other sepsis-associated organ dysfunction, no evidence-based recommendation could be made by the panel. However, in critically ill children, a stepwise approach to increasing enteral feeds has been shown to reduce time needed to reach nutritional goals [364-367]. In our practice, $60 \%$ of panel members always or often and $20 \%$ sometimes commence early enteral feeding within $48 \mathrm{~h}$ of admission in children with septic shock or sepsis-associated organ dysfunction who have no contraindications to enteral nutrition, while $20 \%$ of panel members rarely or never pursue this practice.

\section{We suggest not withholding enteral feeding solely on the basis of vasoactive-inotropic medication administra- tion (weak recommendation, low quality of evidence).}

Remarks: Enteral feeding is not contraindicated in children with septic shock after adequate hemodynamic resuscitation who no longer require escalating doses of vasoactive agents or in whom weaning of vasoactive agents has started.

Rationale: We reviewed indirect evidence from three observational studies (two retrospective and one prospective) in postoperative/cardiac pediatric populations. These studies reported that enteral feeding was tolerated in patients on nonescalating/weaning doses of vasoactive agents without increased adverse effects or gastrointestinal complications [368-370]. In another study of 339 critically ill children, there was no association between enteral feeding and the development of severe gastrointestinal outcomes such as vomiting, diarrhea, abdominal distension, bleeding, necrotizing enterocolitis, or perforation [368]. However, in the report, the decision to start enteral nutrition may have been biased by the clinical condition of the patient. In a retrospective study of 52 critically ill children, the use of vasoactive medications was not associated with an increase in feeding intolerance or gastrointestinal complications [369]. In a prospective observational study of critically ill children who received postpyloric feeding, 44 of 65 patients (67.7\%) with shock and 284 of 461 patients $(61.6 \%)$ without shock received enteral nutrition within $48 \mathrm{~h}$. Although gastrointestinal complications were more common in children admitted with shock, no association between the incidence of digestive tract complications and early (first $48 \mathrm{~h}$ ) or late administration of postpyloric enteral nutrition was reported [370]. Based on these studies which, while providing indirect evidence, all consistently found that enteral feeding was not associated with harm, we recommend not to withhold enteral nutrition solely because vasoactive-inotropic medications are being used. Current evidence supports starting enteral nutrition in hemodynamically stable patients who are no longer requiring fluid resuscitation or escalating doses of vasoactive agents.

53. We suggest enteral nutrition as the preferred method of feeding and that parenteral nutrition may be withheld 
in the first 7 days of PICU admission in children with septic shock or other sepsis-associated organ dysfunction (weak recommendation, moderate quality of evidence).

Rationale: No studies have been published on this specific issue of nutrition in children with septic shock or other sepsis-associated organ dysfunction. However, in a general cohort of 1440 critically ill children enrolled in the international multicenter RCT of pediatric early versus late PN in critical illness [371], withholding parenteral nutrition during the first week in PICU when enteral nutrition was less than $80 \%$ of prescribed goal was clinically superior to providing supplemental parental nutrition within $24 \mathrm{~h}$ of admission [372]. Secondary analyses of the PEPaNIC trial showed that withholding PN was also beneficial in term neonates and children who were undernourished at admission [373, 374], although withholding parenteral nutrition in term neonates was also associated with increased risk of severe hypoglycemia [373]. A long-term follow-up 2 years after PICU admission showed that withholding parenteral nutrition for 1 week did not affect survival, anthropometrics, or health status, but did improve certain domains of neurocognitive development [375]. Although the results of the PEPaNIC trial corroborated the findings from adult RCTs, the optimal timing of parenteral nutrition in the critically ill child with sepsis is still not clear [371, 376-378]. Our recommendation is based on one trial, and therefore, the evidence to withhold PN in the first 7 days of PICU admission is of moderate certainty and must be explored further using pragmatic timing for PN in the first week, particularly in severely malnourished patients and neonates.

54. We suggest against supplementation with specialized lipid emulsions in children with septic shock or other sepsis-associated organ dysfunction (weak recommendation, very low quality of evidence).

Rationale: In two RCTs evaluating immunomodulatory formulas, including lipid emulsions, in critically ill children, outcomes were not significantly different [379, 380]. One RCT was terminated during interim analysis because of unlikely benefit in the intervention arm [380]. In another small RCT, use of enteral feeding supplemented with or without omega-3 fatty acids in 120 critically ill children with sepsis was investigated [381]. Univariate analyses showed a significant difference in inflammatory mediators and reduction in PICU LOS, but these outcome benefits were not evident in the multivariable analyses. Taken together, although promising, insufficient evidence is available to support routine supplementation in pediatric sepsis with specialized lipid emulsions.

55. We suggest against the routine measurements of gastric residual volumes (GRVs) in children with septic shock or other sepsis-associated organ dysfunction (weak recommendation, low quality of evidence).

Rationale: Although routine measurement of GRV is a relatively common practice in PICUs, there is no direct evidence in pediatric sepsis. In a two-center observational cohort study of critically ill children admitted with a variety of diagnoses, one center reported routine use of GRV monitoring while the other center did not practice GRV measurements [382]. The center that advanced enteral nutrition without routine measurements of GRV did not have an increase in the incidence of vomiting, ventilator acquired pneumonia, or necrotizing enterocolitis in comparison with the other PICU (Supplemental Table 21, Supplemental Digital Content 1, http://links.lww.com/PCC/B139). Although there are likely some children for whom measuring GRV would likely be useful (e.g., gastroparesis, omphalocele, gastroschisis), no evidence supports routine measurements in all patients at this time and, if measured, GRV is not sufficient to diagnose EN intolerance.

56. We suggest administering enteral feeds through a gastric tube, rather than a postpyloric feeding tube, to children with septic shock or other sepsis-associated organ dysfunction who have no contraindications to enteral feeding (weak recommendation, low quality of evidence).

Rationale: In three small RCTs, gastric versus postpyloric enteral feeding were compared in mechanically-ventilated children with a variety of diagnoses [383-385]. The outcomes reported included lower caloric achievement with gastric feeding and delayed start of enteral feeding with postpyloric feeding [383, 384]. No significant difference was found in the incidence of ventilator-associated pneumonia between gastric and postpyloric feeding [385]. On the basis of these studies, there is no clear evidence that postpyloric feeding is beneficial and there is concern for potential harm through delayed optimization of enteral nutrition. Therefore, we suggest that feeding with a gastric tube is physiologic and, based on current evidence, the preferred method for enteral nutrition. Postpyloric feeding may be considered in patients in whom gastric feeding is either contraindicated (e.g., high-risk 
for aspiration) or was not tolerated/advanced, and as a result, nutritional goals were unable to be met.

57. We suggest against the routine use of prokinetic agents for the treatment of feeding intolerance in children with septic shock or other sepsis-associated organ dysfunction (weak recommendation, low quality of evidence).

Rationale: Prokinetic agents, such as metoclopramide and erythromycin, are often used in the PICU in an effort to reduce feeding intolerance [386]. Indirect evidence for this question was provided from the only pediatric randomized control trial, which was a combined intervention of enteral zinc, selenium, glutamine, and IV metoclopramide. In critically ill children, this combined intervention failed to reduce the development of sepsis or incidence of hospital-acquired infection in immunocompetent children, although the intervention including metoclopramide did reduce the rate of hospital-acquired infection and sepsis in immunocompromised children. However, the application of this study to children who already have sepsis is not clear. Prokinetic agents are also not without risk as they have been associated with prolongation of the QT interval and ventricular arrhythmias [387-389]. Further investigation is needed to determine if prokinetic agents are beneficial in patients with sepsis, particularly in immunocompromised children.

58. We suggest against the use of selenium in children with septic shock or other sepsis-associated organ dysfunction (weak recommendation, low quality of evidence).

Rationale: Although clinical research examining the use of selenium among critically ill neonates and adults has been done (Supplemental Table 22, Supplemental Digital Content 1, http://links.lww.com/PCC/B139), there no data regarding selenium supplementation as potential adjunctive therapy for pediatric sepsis. Selenium plays a key role as a cofactor for glutathione peroxidase, iodothyronine deiodinase, and thioredoxin [390]; accordingly, selenium deficiency could affect thyroid metabolism and the response to oxidative stress during critical illness. Furthermore, low serum selenium concentrations are common in critical illness [391, 392] and infection [393], and have been associated with measures of oxidative stress in neonates [394] and adults [395].

A systematic review of investigations examining selenium supplementation in preterm neonates reported improved outcomes, including reduction in occurrence of sepsis [396]. Similarly, a published systematic review and meta-analysis of the effect of parenteral selenium supplementation in critically ill adult sepsis patients concluded that this intervention reduced risk of mortality
[397], but when the meta-analysis was updated to include the results of a more recent RCT, there was no difference in mortality in those treated with or without selenium supplementation [50]. In an interventional trial examining the potential benefit of zinc, selenium, glutamine, and metoclopramide administration to critically ill children, there was no reduction in the primary outcome measure, namely, time until the first episode of nosocomial infection/sepsis [380]. Based on lack of interventional trials examining selenium supplementation in the setting of pediatric sepsis and sepsis-associated organ dysfunction, we suggest against its use as a weak recommendation.

59. We suggest against the use of glutamine supplementation in children with septic shock or other sepsis-associated organ dysfunction (weak recommendation, low quality of evidence).

Rationale: During catabolic stress, the human body is unable to produce adequate quantities of glutamine and, therefore, its essential role as a fuel source for enterocytes and immune cells is diminished. Over the past 2 decades, several investigations of glutamine administration alone and in various combinations with other nutritional supplements have been conducted in critically ill populations [380, 398-404], including those with sepsis [399, 405-407]. Contemporary studies have not found glutamine in any form (enteral or parenteral) and/or in combination with other nutritional elements to significantly improve morbidity or mortality in critically ill infants, children, and adults, including those with sepsis [408-410] (Supplemental Table 23, Supplemental Digital Content 1, http://links.lww.com/PCC/B139). However, single-element studies administering only glutamine to children with sepsis and septic shock are scarce. An RCT by Jordan et al. [401] randomized children ( 49 control; 49 interventional) with sepsis and septic shock requiring PN to either standard PN or PN with glutamine supplementation for the purpose of examining oxidative stress and inflammatory response. This investigation supports earlier studies in broader populations finding no differences in PICU $(p=0.062)$ or hospital LOS $(p=0.09)$ or hospital mortality $(\mathrm{p}=0.31)$. Two other studies of glutamine administration in combination with other elements to children with septic shock and critical illness are available [380, 399]. The RCT by Briassoulis et al. [399] examined children with septic shock receiving glutamine in combination with arginine, antioxidants, and omega-3 fatty acids. Although the main outcome of change in cytokines showed some promise, no difference was noted between groups for hospital survival ( $80 \%$ vs $87 \%$ ) or LOS $(10.4 \pm 2.2$ vs $11.4 \pm 2.5$ days) [15]. Carcillo et al. [380] randomized 283 subjects from eight PICUs to a control 
group receiving whey protein formula or an intervention group receiving formula with zinc, selenium, glutamine, and IV metoclopramide supplementation. There was no difference between hospital-acquired infections and clinical sepsis per 100 days $(\mathrm{p}=0.81)$, PICU LOS $(\mathrm{p}=0.16)$, or 28-day mortality (8/139 [5.8\%] vs $15 / 145$ [10.3\%]). Subjects from this trial were also categorized by immune status with the suggestion that immune status may play a role in the effectiveness of nutritional supplemental, including glutamine [411]. However, no direct evidence regarding glutamine supplementation in children with sepsis exists; hence, we suggest against the use of glutamine therapy in children with septic shock or other sepsis-associated organ dysfunction until further data become available.

60. We suggest against the use of arginine in the treatment of children with septic shock or other sepsis-associated organ dysfunction (weak recommendation, very low quality of evidence).

Rationale: Reduced availability of arginine in sepsis may lead to decreased endogenous nitric oxide synthesis, loss of microcirculatory regulation, and altered immune response [412-414]. In the only pediatric RCT of arginine supplementation in children with sepsis [415], 10 children received infusions of arginine and had enhanced arginine oxidation and increased nitric oxide levels, but no clinical outcomes were reported. In indirect data from adult studies, RCTs of L-arginine supplementation have been small and have reported both positive and negative effects on mortality [416-420]. One trial in septic adults found decreased mortality [418], but other studies found no benefit or increased mortality in adults with sepsis $[416,419,420]$. Some authors found improvement in secondary outcomes in patients with sepsis, such as reduced infectious complications and shorter LOS, but the relevance of these findings and their applicability to children with sepsis in the face of potential harm is unclear. Hence, in the absence of evidence of demonstrated benefit, we suggest against the use of arginine therapy in children with sepsis-associated organ dysfunction until further data become available.

61. We suggest against using zinc supplementation in children with septic shock and other sepsis-associated organ dysfunction (weak recommendation, very low quality of evidence).
Rationale: Alterations in zinc homeostasis and associations between zinc levels and outcomes have been reported in the critically ill. Benefits of zinc supplementation have been shown in some forms of infectious illnesses. However, no trials of zinc supplementation in children with sepsis have been conducted. One RCT in critically ill children comparing daily supplementation with zinc, selenium, glutamine, and metoclopramide versus whey protein was stopped during interim analysis due to futility [380]. Based on conflicting studies in the adult literature, routine supplementation of zinc is not recommended in nutritional guidelines for critically ill adults [421]. Future RCTs examining the optimal timing and dose of zinc in children with sepsis and septic shock and its impact on immune response and clinical outcomes might help answer this question.

62. We suggest against the use of ascorbic acid (vitamin $C$ ) in the treatment of children with septic shock or other sepsis-associated organ dysfunction (weak recommendation, very low quality of evidence).

Rationale: Ascorbic acid (vitamin C) has multiple physiologic functions. Most importantly in the setting of sepsis, vitamin $C$ is an antioxidant and neutralizes reactive oxygen and nitrogen radicals, inhibits activation of pro-inflammatory cytokines, increases endogenous vasopressor synthesis, and inhibits bacterial replication [422-424]. Adults with sepsis frequently have very low levels of vitamin C. In one study, $88 \%$ of adults with septic shock had hypovitaminosis C [425]. Small studies in adults suggest that treatment of septic patients with vitamin $C$ may improve organ dysfunction [426] and reduce mortality [427]. Vitamin $C$ has also been used as a component of combination therapy, typically with thiamine and corticosteroids, in adults with sepsis [428]. One study compared such treatment in 47 adult patients with sepsis to historical control patients [429]. Treatment was associated with decreased hospital mortality (OR, 0.13; 95\% CI, 0.04-0.48), shorter duration of vasopressor therapy, and improved organ dysfunction scores (Supplemental Table 24, Supplemental Digital Content 1, http://links.lww.com/PCC/ B139).

Currently, there are no data on the use of vitamin $\mathrm{C}$ in critically ill children or in pediatric sepsis. The prevalence of low vitamin $C$ levels in septic children is unknown, and no studies have investigated the effect of vitamin $C$ supplementation, either alone or in combination with other agents, in the treatment of pediatric sepsis. 
63. We suggest against the use of thiamine to treat children with sepsis-associated organ dysfunction (weak recommendation, low quality of evidence).

Rationale: Thiamine is a crucial factor in cellular metabolism. In its active form, thiamine pyrophosphate is an essential coenzyme used to generate energy (adenosine triphosphate) from glucose. The human body does not produce thiamine and, with a short half-life and small body stores, thiamine deficiency can develop within days of critical illness and inadequate nutrition, resulting in impaired oxidative and carbohydrate metabolism. Low blood concentrations of thiamine have been reported at admission of critically ill children and adults with sepsis and septic shock [430-432]. A study examining thiamine deficiency in children admitted to the PICU showed that low blood thiamine concentration in those with severe sepsis or septic shock was associated with mortality (OR, 8.40; 95\% CI, 1.38-51.0) [431]. In an RCT of 88 adults with septic shock (Supplemental Table 25, Supplemental Digital Content 1, http://links.lww.com/PCC/B139), there were no differences between treatment with thiamine versus placebo for the primary outcome of change in lactate levels or the secondary outcomes of mortality, shock reversal, and LOS [430]. However, on post hoc analysis, thiamine treatment in the subgroup with thiamine deficiency at admission was associated with lower lactate level within $24 \mathrm{~h}$ and lower mortality $(\mathrm{p}=0.047)$. However, more evidence is needed to recommend whether thiamine supplementation should be used to treat children with septic shock or other sepsis-associated organ dysfunction. Also, it may be important for this evidence to be considered in the context of thiamine status at PICU admission.

64. We suggest against the acute repletion of vitamin $D$ deficiency (VDD) for treatment of septic shock or other sepsis-associated organ dysfunction (weak recommendation, very low quality of evidence).

Rationale: A systematic review and meta-analysis of 17 studies including 2783 patients showed that approximately half of critically ill children have VDD (25-hydroxy vitamin D level $<50 \mathrm{nmol} / \mathrm{L}$ or $<20 \mathrm{ng} / \mathrm{mL}$ ) at PICU admission [189]. Further, VDD was associated with higher illness severity, multiple organ dysfunction, and mortality across these studies. Six of these studies focused on or separately analyzed children with sepsis [433-437]. Three studies reported a greater need for vasoactive agents in VDD children [433-435], although mortality across these six studies was not associated with VDD [433-437] (Supplemental Table 26, Supplemental Digital Content 1, http://links.lww.com/PCC/B139).
Vitamin D levels are lowered by fluid resuscitation, which can confound the association with illness severity and disease complications [434]. In addition, free or bioavailable 1,25(OH)2D is the active form which is influenced by the level of vitamin D binding protein (VDBP) and a patient's VDBP genotype, which was not estimated or measured in prior studies [438]. Although vitamin D levels are a potentially modifiable risk factor via supplementation, a meta-analysis of rapid normalization of vitamin D levels concluded that it is best achieved using loading therapy that takes into account disease status, determines baseline vitamin D level, and considers patient weight [439-441]. A loading dose greater than 300,000 international units should be avoided outside of RCTs evaluating risk and benefit.

Hypervitaminosis D is associated with hypercalcemia and other severe complications [442] and vitamin D overdoses can be fatal [443]. No current data support that rapid acute correction of VDD is an effective treatment in septic shock or improves outcomes of septic children. Further, measurement of $25(\mathrm{OH}) 2 \mathrm{D}$ levels is not currently a standard component of sepsis care and methods of accurately measuring bioavailable vitamin $\mathrm{D}$ are not yet widely validated. However, if VDD is diagnosed, repletion should occur as a usual part of general holistic pediatric care according to recommended guidelines independently of the presence of sepsis [444].

\section{Blood products}

65. We suggest against transfusion of $R B C s$ if the blood hemoglobin concentration is greater than or equal to $7 \mathrm{~g} / \mathrm{dL}$ in hemodynamically stabilized children with septic shock or other sepsis-associated organ dysfunction (weak recommendation, low quality of evidence).

Remarks: According to the 2018 Transfusion and Anemia Expertise Initiative (TAXI) guidelines, for the purposes of RBC transfusion, "hemodynamically stabilized" is defined as a MAP higher than 2 sds below normal for age and no increase in vasoactive medications for at least $2 \mathrm{~h}$.

66. We cannot make a recommendation regarding hemoglobin transfusion thresholds for critically ill children with unstable septic shock.

Rationale: The only study evaluating specific RBC transfusion thresholds in children with sepsis is a post hoc subgroup analysis of the Transfusion Requirements in the PICU (TRIPICU) study [445] (Supplemental Table 27, Supplemental Digital Content 1, http://links .lww.com/PCC/B139). This study included 137 stabilized critically ill children (MAP $>2$ sds below normal for age 
and cardiovascular support not increased for at least $2 \mathrm{~h}$ before enrollment) with sepsis, with a hemoglobin less than or equal to $9.5 \mathrm{~g} / \mathrm{dL}$ within 7 days after PICU admission. Patients were randomized to receive RBCs if hemoglobin decreased to either less than $7.0 \mathrm{~g} / \mathrm{dL}$ (restrictive group) or $9.5 \mathrm{~g} / \mathrm{dL}$ (liberal group). No differences were found between the restrictive versus liberal group in the primary endpoint of new or progressive multiple organ dysfunction syndrome $(18.8 \%$ vs $19.1 \%)$ or mortality $(p=0.44)$. These results are similar to those from primary analysis of the TRIPICU study [446], as well as in adults [447]. Our suggestion against transfusion if hemoglobin is greater than $7 \mathrm{~g} / \mathrm{dL}$ in hemodynamically stable children with sepsis parallels the TAXI recommendations [448].

Insufficient data are available to guide $\mathrm{RBC}$ transfusion therapy in children with unstable septic shock. Two pediatric RCTs did demonstrate decreased mortality when red blood transfusion to goal hemoglobin greater than or equal to 10 (hematocrit $>30 \%$ ) was included as part of an EGDT algorithm targeting Scvo2, but the impact of each individual component, including red blood transfusion, is unclear [190, 449]. In critically ill adults, the Transfusion Requirements in Septic Shock trial randomized 998 subjects with septic shock to either a transfusion threshold hemoglobin of $7 \mathrm{~g} / \mathrm{dL}$ or $9 \mathrm{~g} / \mathrm{dL}$ [450]. At randomization, all patients had hypotension (mean arterial pressure $<70 \mathrm{~mm} \mathrm{Hg}$ ) and/or were being treated with vasopressors. Ninety-day mortality showed no differences (RR, 0.94; 95\% CI, 0.78-1.09), suggesting that a restrictive transfusion strategy in hemodynamically unstable septic adults was safe (Supplemental Table 27, Supplemental Digital Content 1, http://links.lww.com/ $\mathrm{PCC} / \mathrm{B} 139)$. The SSC recommends that RBC transfusion in adults occur only when hemoglobin concentration decreases to less than $7.0 \mathrm{~g} / \mathrm{dL}$ in the absence of extenuating circumstances, such as myocardial ischemia, severe hypoxemia, or acute hemorrhage (strong recommendation, high quality of evidence) [50]. This adult recommendation is also valid for hemodynamically unstable patients.

However, in the absence of pediatric data, we are not able to provide a recommendation for critically ill children with unstable septic shock.

67. We suggest against prophylactic platelet transfusion based solely on platelet levels in nonbleeding children with septic shock or other sepsis-associated organ dysfunction and thrombocytopenia (weak recommendation, very low quality of evidence).

Rationale: One observational study demonstrated an association between the administration of platelet transfusions to critically ill children and worse clinical outcomes (Supplemental Table 28, Supplemental Digital Content 1, http://links.lww.com/PCC/B139), including longer ICU LOS, progressive organ dysfunction, and increased mortality [451]. Indirect evidence can be found in an RCT of 660 infants born at less than 34 weeks gestational age, the majority of whom were treated for sepsis, that compared a platelet transfusion threshold of 50,000/ $\mathrm{mm} 3$ (high threshold) with $25,000 / \mathrm{mm} 3$ (low threshold) [452]. More infants in the high- versus low-threshold group received at least one platelet transfusion $(90 \%$ vs $53 \%)$. More adverse events, including new major bleeding or death, were also seen in the high threshold group (OR, 1.57 ; 95\% CI, 1.06-2.32).

Although existing evidence does not support a platelet threshold at which transfusion is absolutely indicated, the risk of spontaneous bleeding may be greater at lower platelet counts, for example, less than 10-20,000/ $\mathrm{mm} 3$. In addition, some populations of thrombocytopenic critically ill children may have a relatively high risk of bleeding, such as those with oncological diagnoses or those receiving ECMO. Because the threshold at which the benefits of platelet transfusion outweigh the risks is unknown, clinical judgment based on patient risk factors for bleeding in addition to the measured platelet level must be exercised carefully.

68. We suggest against prophylactic plasma transfusion in nonbleeding children with septic shock or other sepsisassociated organ dysfunction and coagulation abnormalities (weak recommendation, very low quality of evidence).

Remarks: Prophylactic plasma transfusion refers to situations in which there is an abnormality in laboratory coagulation testing but no active bleeding.

Rationale: No direct data exist to inform a recommendation about plasma transfusion in pediatric sepsis. One RCT evaluates prophylactic plasma transfusion in critically ill children without sepsis. Pieters et al. [453] randomized 81 children less than 2 years old requiring primary repair of craniosynostosis to receive plasma using either a prophylactic (in absence of bleeding) or reactive (when the patient was bleeding) strategy. The prophylactic plasma transfusion group received a significantly higher volume of plasma compared with the reactive group ( $29.7 \mathrm{vs} 16.1 \mathrm{~mL} / \mathrm{kg} ; \mathrm{p}<0.001)$. Despite an improvement in coagulation values in the prophylactic group, there was no difference in packed RBC transfusion requirements or blood loss between the two groups (Supplemental Table 29, Supplemental Digital Content 1, http://links.lww.com/PCC/B139). Additionally, a meta-analysis published in 2012 that included 80 RCTs 
(mostly in adults) concluded that there was no consistent evidence for benefit of prophylactic plasma transfusion across a range of indications that were evaluated [454]. Observational studies in critically ill children have shown that plasma transfusions are associated with worse clinical outcomes [455, 456]. Furthermore, plasma transfusion frequently fails to correct abnormal coagulation tests in critically ill adults and children [456, 457]. We therefore suggest against prophylactic plasma transfusions for children with septic shock and other sepsis-associated organ dysfunction who are not bleeding.

However, some specific patient populations might benefit from prophylactic plasma transfusions, such as patients with worsening coagulation tests at high risk for disseminated intravascular coagulopathy, children with comorbid cancer, or children with sepsis on extracorporeal life support.

\section{Plasma exchange, renal replacement, and extracorporeal support}

69. We suggest against using plasma exchange (PLEX) in children with septic shock or other sepsis-associated organ dysfunction without thrombocytopenia-associated multiple organ failure (TAMOF) (weak recommendation, very low quality of evidence).

70. We cannot suggest for or against the use of PLEX in children with septic shock or other sepsis-associated organ dysfunction with TAMOF.

Rationale: Therapeutic PLEX for septic shock or sepsis-associated organ dysfunction aims to normalize the plasma milieu of a systemically inflamed septic patient. Currently, no large RCTs have evaluated PLEX in pediatric septic shock or sepsis-associated organ dysfunction. Rimmer et al. [458] performed a meta-analysis that included four small RCTs evaluating PLEX in adults $(\mathrm{n}=128)$ and pediatric $(\mathrm{n}=66)$ patients with sepsis and septic shock. PLEX was associated with reduced mortality in adults (RR, 0.63; 95\% CI, 0.42-0.96), but not in children (RR, 0.96; 95\% CI, 0.28-3.38). However, because of the heterogeneity of the patient population, inclusion criteria, technical modalities of PLEX (filtration vs centrifugation), and types of replacement fluid (plasma vs albumin) in these four studies as well as the costs and potential risks, PLEX cannot be routinely recommended as this time (Supplemental Table 30, Supplemental Digital Content 1, http://links.lww.com/PCC/B139). Similarly, the American Society for Apheresis recommended that the "optimum role of apheresis therapy is not established" in sepsis with multiple organ failure [459].

TAMOF is an inflammatory phenotype of sepsisinduced multiple organ dysfunction in children that can be identified clinically by new-onset thrombocytopenia and evolving multiple organ dysfunction [460, 461]. Autopsies performed on patients who died with TAMOF revealed disseminated microvascular thromboses in various organs [460]. These patients had deficient activity of a disintegrin and metalloproteinase with thrombospondin type 1 motif (ADAMTS-13), elevated von Willebrand factor (VWF) activity, and the presence of ultra-large plasma VWF [460, 462]. Decreased activity of ADAMTS-13 leads to high circulating levels of ultralarge VWF that induce widespread platelet activation and thrombotic microangiopathy. A number of inflammatory mediators are elevated in sepsis that can inhibit or inactivate ADAMTS-13 including interleukin-6, granulocyte elastase, plasmin, thrombin, cell-free hemoglobin, Shiga toxins, and immunoglobulin G auto-antibody [463-468].

Three studies have examined the utility of PLEX in children with sepsis and TAMOF [460, 469, 470]. In the most recent and largest study $(n=81)$, Fortenberry et al. [469] reported that PLEX was associated with lower 28-day mortality by multivariate analysis (adjusted RR, 0.45; 95\% CI, 0.23-0.90) and by propensity score weighting (adjusted RR, 0.46; 95\% CI, 0.220.97). In a retrospective cohort study from the Turkish TAMOF Network $(\mathrm{n}=42)$, PLEX was associated with lower 28-day mortality compared with the no PLEX group (27\% vs $70 \% ; p=0.004)$ [470]). In the third study, Nguyen et al. [460] randomized 10 children to either PLEX or standard therapy. The 5 patients who received PLEX had restoration of ADAMTS-13 activity and greater survival (5/5) compared with standard therapy $(1 / 5 ; \mathrm{p}<0.05)$. Taken together, these data support a biologic rationale for the use of PLEX in TAMOF, that is, the removal of pathologic ultra-large VWF and ADAMTS-13 inhibitors and restoration of ADAMTS-13 activity. This approach of using PLEX is similar to the rationale for using PLEX in thrombotic thrombocytopenic purpura [471]. Although the panel acknowledges a potential benefit for PLEX and encourages an RCT to better define the utility of PLEX in children with sepsis and TAMOF, a recommendation could not be made based on existing data.

71. We suggest using renal replacement therapy to prevent or treat fluid overload in children with septic shock or other sepsis-associated organ dysfunction who are unresponsive to fluid restriction and diuretic therapy (weak recommendation, very low quality of evidence).

Rationale: Renal replacement therapy is increasingly being used in PICUs for renal and nonrenal conditions. The rationale for renal replacement therapy in septic shock includes impending or established fluid overload following initial resuscitation or for cytokine removal, 
reversal of coagulopathy, to buffer lactic acidosis, to address AKI, or a combination of these factors. CRRT may be useful for treating established fluid overload or to prevent further fluid overload while allowing liberal volume administration for nutrition, antimicrobials, and other medications, sedation, and transfusions. In addition, certain techniques of continuous blood purification may help to regulate systemic inflammation and promote kidney recovery [472]. Fluid overload has been shown to cause increased morbidity and mortality in various intensive care settings, and there is documented favorable association of CRRT in fluid overload [473].

However, no high-quality studies in critically ill children with sepsis exist to directly determine whether RRT is definitively beneficial compared with diuretics and/or fluid restriction. Most of the data come from adult studies where outcomes have varied from mortality to ICU LOS and ventilator- and vasoactive-free days. One study addressed the timing of CRRT initiation in 27 children with sepsis and multiple organ dysfunction, demonstrating that CRRT was associated with survival when started within $48 \mathrm{~h}$ of admission compared with those started on CRRT after $48 \mathrm{~h}$ of admission ( $61 \%$ vs $33 \%$; $\mathrm{p}<0.001)$. However, timing of CRRT initiation was at the discretion of the treating team, raising concern for confounding between groups, and all patients in both groups experienced normalization of kidney function [474] (Supplemental Table 31, Supplemental Digital Content 1, http:// links.lww.com/PCC/B139).

The possible benefits of CRRT must also be weighed against potential risks, including the need for an invasive catheter, costs, limited availability in some centers, the need for clinician and nursing-specialist expertise, and the challenge of optimal timing (e.g., following resuscitation for fluid removal or earlier for acute cytokine clearance). Therefore, as the initial treatment strategy, we judge that fluid restriction and use of diuretics are reasonable in the presence of impending or established fluid overload with CRRT reserved as a second-line option to prevent or treat fluid overload in children with septic shock or other sepsis-associated organ dysfunction who are unresponsive to fluid restriction and diuretic therapy.

72. We suggest against high-volume hemofiltration (HVHF) over standard hemofiltration in children with septic shock or other sepsis-associated organ dysfunction who are treated with renal replacement therapy (weak recommendation, low quality of evidence).

Rationale: HVHF for critically ill patients with septic shock and AKI is an appealing strategy for maintaining acid-base and fluid homeostasis, or for having a potential immunomodulatory effect in sepsis by removal of toxins and other inflammatory mediators, especially cytokines that contribute to organ injury and dysfunction.

In adults, use of higher CRRT flux rates $(>35 \mathrm{~mL} / \mathrm{kg} /$ hr filtration-dialysis), while initially encouraging, has not shown overall mortality benefit in subsequent RCTs and meta-analysis. A 2017 Cochrane review found no significant benefit in mortality, severity of organ dysfunction, LOS, or adverse effects with HVHF versus standard hemofiltration rates in critically ill adults [475]. Notably, the results of this meta-analysis show that very few studies have been conducted to investigate the use of HVHF in critically ill patients with septic shock (four studies totaling 201 participants).

In a study involving 155 pediatric patients with severe sepsis, HVHF treatment did not significantly reduce 28-day mortality compared with standard volume CRRT. Furthermore, there were no significant reductions in plasma levels of inflammatory mediators or in improving hemodynamic variables for HVHF. However, the incidence of hyperglycemia was significantly higher in HVHF group than in CVVH group [476] (Supplemental Table 32, Supplemental Digital Content 1, http://links .lww.com/PCC/B139).

\section{We suggest using venovenous ECMO in children with sepsis-induced PARDS and refractory hypoxia (weak recommendation, very low quality of evidence).}

Rationale: ECMO was introduced more than 40 years ago to support patients with reversible but severe cardiovascular and/or respiratory failure refractory to conventional medical therapy. As such, children with life-threatening sepsis-induced ARDS are often considered as candidates for ECMO rescue [477], and PALICC endorsed ECMO for the treatment of refractory hypoxia [24]. The use of ECMO in pediatric sepsis has increased over the past decade [478, 479]; whether this has improved survival remains to be determined [480]. To date, no RCT examining the effect of ECMO on outcome in pediatric sepsis has been published. In the absence of such data, using propensity score matching, Barbaro et al. [481] reported that children with severe PARDS enrolled in the RESTORE trial had similar mortality rates when supported with ECMO $(15 / 61,25 \%)$ as compared with those who were not $(18 / 61,30 \%)$ [482] (Supplemental Table 33, Supplemental Digital Content 1, http://links lww.com/PCC/B139). Research is underway to determine optimal pre-ECMO candidacy [483] as measures of renal, hepatic, neurologic, and hematologic dysfunction, and particularly the presence of bloodstream infections, seem to discriminate mortality risk better than traditional pediatric severity of illness scores such as PRISM, Pediatric Index of Mortality, and Pediatric Logistic Organ 
Dysfunction. Clearly, ECMO is not available worldwide, and transfer of highly unstable patients to higher levels of care that offer the therapy can carry substantial risk. However, adult and pediatric data suggest a potential association with improved mortality, particularly if transfer is to high volume ECMO centers $[484,485]$.

74. We suggest using venoarterial ECMO as a rescue therapy in children with septic shock only if refractory to all other treatments (weak recommendation, very low quality of evidence).

Rationale: Several anecdotal reports of use of venoarterial ECMO in the management of RSS in children exist. More recent reports suggest that venoarterial ECMO may be associated with better survival than conventional therapy, and strategies to maximize flow rates to reverse shock and multiple organ dysfunction may play an important role $[486,487]$. However, considerable concern surrounds the risks of this highly invasive therapy, such as hemorrhage and thromboembolic events.

The most recent and largest report of venoarterial ECMO in 44 pediatric patients with RSS secondary to bacterial, viral, or fungal infection admitted to seven tertiary PICUs across five different countries compared their outcome to 120 children with RSS managed by conventional therapy [488]. Inclusion in the study required children to meet three of four criteria for severe septic shock in the first $24 \mathrm{~h}$ of their ICU stay: arterial $\mathrm{pH}$ less than or equal to 7.15, arterial lactate greater than or equal to $4.0 \mathrm{mmol} / \mathrm{L}$, base excess less than or equal to $-10 \mathrm{mmol} / \mathrm{L}$, and in-hospital cardiac arrest. Patients were excluded if they had cyanotic congenital heart disease, myocarditis, or an out-of-hospital cardiac arrest. The results showed no significant difference in survival to hospital discharge (50\% in the venoarterial ECMO cohort vs $40 \%$ in the conventional therapy cohort). Survival was significantly higher in patients who received high ECMO flows ( $>150 \mathrm{~mL} / \mathrm{kg} / \mathrm{min}$ at $4 \mathrm{~h}$ after institution of ECMO) compared with children who received standard ECMO flows or no ECMO (Supplemental Table 34, Supplemental Digital Content 1, http://links.lww.com/PCC/B139).

The potential use of venoarterial ECMO for RSS suggests that the definition of RSS should be standardized across institutions. As yet, no universal definition of RSS in children exists. One published definition that could be applied is from the European Society of Paediatric and Neonatal Intensive Care [10]. The suggested definition for RSS was blood lactate greater than $8 \mathrm{mmol} / \mathrm{L}$ or a $1 \mathrm{mmol} / \mathrm{L}$ lactate increase after $6 \mathrm{~h}$ of resuscitation and high vasoactive dependency (vasopressor-inotrope score $>200$ ), or myocardial dysfunction defined as the occurrence of a resuscitation-responsive cardiac arrest in PICU or cardiac ultrasound findings with left ventricle ejection fraction less than $25 \%$ or a cardiac index less than $2.2 \mathrm{~L} / \mathrm{min} / \mathrm{m}^{2}$.

\section{Immunoglobulins}

75. We suggest against the routine use of IV immune globulin (IVIG) in children with septic shock or other sepsis-associated organ dysfunction (weak recommendation, low quality of evidence).

Remarks: Although routine use of IVIG is not recommended, select patients may benefit from such treatment.

Rationale: The proposed rationale for IVIG in severe infections is to boost passive immunity through neutralization of bacterial toxins, promoting opsonization of bacteria, and inhibition of immune cell proliferation and inflammatory mediators. However, IVIG has considerable batch-tobatch variability and its true biologic activity is not clear. There are no high-quality studies of IVIG in critically ill children with sepsis, and small observational studies have reported conflicting results [489]. An RCT of polyclonal IVIG in 100 children with sepsis demonstrated a reduction in mortality (28\% vs 44\%), LOS (6 vs 9 days), and less progression to complications ( $8 \%$ vs $32 \%$ ) [490]. However, a more recent multicenter trial of polyclonal IVIG in 3493 neonates with suspected or proven serious infection found no significant differences in mortality or major disability [491]. Other studies have been carried out with specific monoclonal antibodies (e.g., monoclonal antibody against endotoxin in children with meningococcal septic shock), but there are no definitive data to support general benefit of polyclonal immunoglobulin in neonates or children with septic shock at this time. Data from adult patients with septic shock also do not support a routine benefit of IVIG [492], although administration of immunoglobulin M- and immunoglobulin A-enriched polyclonal IVIG has shown possible efficacy [493] (Supplemental Table 35, Supplemental Digital Content 1, http://links.lww.com/PCC/B139).

For patients with toxic shock syndrome, especially those with streptococcal etiology, polyclonal IVIG may have clinical utility [494]. Other potential pediatric populations that may benefit from IVIG in sepsis are those with necrotizing fasciitis (although evidence in adults does not support use $[495,496])$, and those with primary humoral immunodeficiencies or immunocompromised with documented low immunoglobulin levels.

\section{Prophylaxis}

76. We suggest against the routine use of stress ulcer prophylaxis in critically ill children with septic shock or other sepsis-associated organ dysfunction, except 
for high-risk patients (weak recommendation, very low quality of evidence).

Remarks: Although routine stress-ulcer prophylaxis is not recommended, some high-risk patients may benefit from stress ulcer prophylaxis. Studies have supported benefit of stress-ulcer prophylaxis when baseline rate of clinically important bleeding is approximately $13 \%$.

Rationale: Stress ulcer prophylaxis should not be routinely administered to children with septic shock or other sepsis-associated organ dysfunction, as evidence for benefit is lacking [497] and may increase risk of adverse effects, such as pneumonia or C. difficile (formerly Clostridium) infection [498]. Rather than routine, universal administration of stress-ulcer prophylaxis, individual patients should be assessed for the presence of risk factors of clinically important gastrointestinal bleeding. These include multiple organ dysfunction [499], prolonged mechanical ventilation ( $>48 \mathrm{~h}$ ), coagulopathy, persistent shock, and treatment with corticosteroids and nonsteroidal anti-inflammatory agents [500].

The risk of GI bleeding is also reduced by mucosal protection introduced by gastric feeding. Early enteral nutrition could therefore be a viable alternative to pharmacological stress-ulcer prophylaxis. A meta-analysis of 1836 adult patients reported that, in the presence of enteral nutrition, pharmacological stress ulcer prophylaxis did not significantly change the risk of GI bleeding. Notably, in those patients who received enteral nutrition and were treated with stress ulcer prophylaxis, the risk of pneumonia was increased compared with patients not treated with stress ulcer prophylaxis (OR, 2.81; 95\% CI, 1.2-6.6) [501] (Supplemental Table 36, Supplemental Digital Content 1, http://links.lww.com/PCC/B139).

77. We suggest against routine deep vein thrombosis (DVT) prophylaxis (mechanical or pharmacologic) in critically ill children with septic shock or other sepsisassociated organ dysfunction, but potential benefits may outweigh risks and costs in specific populations (weak recommendation, low quality of evidence).

Rationale: An open-label RCT of low molecular weight heparin to prevent CVC-associated thrombosis in the PICU was terminated early because of poor recruitment [502]. Eleven of 78 patients (14.1\%) randomized to reviparin had DVT proven on venogram versus 10 of 80 controls (12.5\%) (OR, 1.15; 95\% CI, 0.42-3.23). Three adverse events (major bleed or death) all occurred in the control group and no deaths occurred because of venous thromboembolism (Supplemental Table 37, Supplemental Digital Content 1, http://links.lww.com/PCC/B139).
A subsequent systematic review found the quality of evidence to be low and that the efficacy of low molecular weight heparin in preventing $\mathrm{CVC}$-associated thrombosis is unknown [503]. It is important to highlight that these studies were specific to children with CVCs who may or may not have had sepsis and that they may not apply to the general thromboembolic risk in children with sepsis.

Although CVCs represent the principal risk factor for DVT in infants [504], older children may have other risk factors. For example, the risk of DVT increases in adolescence, obesity, cancer, and in those with multiple medical conditions, especially renal and cardiac disease [505, 506]. At present, it is unknown whether certain high-risk populations of children with sepsis may benefit from DVT prophylaxis.

\section{Knowledge gaps and research opportunities}

This report from the SSC pediatric guidelines panel covers five main topic areas (i.e., early recognition and infection, hemodynamics, ventilation, endocrine and metabolic therapies, and adjunctive therapies) with a total of 77 recommendations arising from 67 PICO questions. On review of these evidence-based analyses, it is clear that, for many PICO questions, the literature review failed to identify sufficient data to develop strong (or even weak in some instances) recommendations for critically ill children with septic shock or other sepsis-associated organ dysfunction. These SSC pediatric guidelines, at the same time, also identified gaps that can inform future research opportunities. As new research populates the evidencebase, it can then be used to develop future iterations of the SSC pediatric guidelines, creating a cycle designed to grow the evidence and increase the number of strong recommendations in the future. Further clarity is needed from both informative pathophysiology studies as well as well-designed RCTs, and the panelists have listed these in the text. The design of meaningful and effective future research should be informed by the needs identified by the collective clinical expertise within the panel.

Overall, the process of developing the SSC-pediatric guidelines generated at least 29 pathophysiology questions warranting further study and 23 RCTs (i.e., total of 52 studies). We present these questions as research opportunities, but have not yet prioritized these opportunities into a formal research agenda (Table 6). We envision that many of the pathophysiology questions can be taken up by individual research groups, and we hope that the SSC children's guidelines document will serve as a template of current evidence and how best to fill the gaps in our knowledge. In contrast, the necessary RCTs will need a coordinated national/international effort and our community will need to prioritize the most appropriate 
Table 6 Knowledge gaps and research opportunities [refer to numbered recommendations in guidelines and Appendix 1

\begin{tabular}{|c|c|c|}
\hline Subgroup & Pathophysiology & Clinical trials \\
\hline \multirow{4}{*}{$\begin{array}{l}\text { Screening, diagnosis, and systematic } \\
\text { management of sepsis: four patho- } \\
\text { physiology studies and two RCTs }\end{array}$} & $\begin{array}{l}\text { Ql screening tool algorithms to recognize clinical deterioration } \\
\text { (see Rec 1) }\end{array}$ & Pediatric sepsis recognition (see Rec 1) \\
\hline & Define the optimal level of hyperlactatemia (see Rec 2) & \multirow{3}{*}{$\begin{array}{l}\text { Initial or serial measurement of blood lactate } \\
\text { directly informs evaluation and/or manage- } \\
\text { ment (see Rec 2) }\end{array}$} \\
\hline & Protocol/guideline for management (see Rec 3) & \\
\hline & $\begin{array}{l}\text { New molecular technologies in identifying pathogens before } \\
\text { blood culture positivity or after antibiotic administration (see } \\
\text { Rec 4) }\end{array}$ & \\
\hline \multirow[t]{7}{*}{$\begin{array}{l}\text { Antimicrobial therapy: seven patho- } \\
\text { physiology studies }\end{array}$} & $\begin{array}{l}\text { The definition of "timely" antimicrobials in a bundle of initial care } \\
\text { (see Recs } 5 \text { and 6) }\end{array}$ & \\
\hline & QI metrics to assess unnecessary antimicrobials (see Rec 7) & \\
\hline & $\begin{array}{l}\text { Antimicrobial resistance rate thresholds to help decide when the } \\
\text { addition of a glycopeptide or second gram-negative agent is } \\
\text { necessary (see Rec } 10 \text { and 11) }\end{array}$ & \\
\hline & $\begin{array}{l}\text { Alteration in the pharmacokinetics and pharmacodynamics of } \\
\text { antimicrobials (see Rec 12) }\end{array}$ & \\
\hline & $\begin{array}{l}\text { The relationship between antimicrobial stewardship programs } \\
\text { and a decrease in antimicrobial resistance (see Rec 13) }\end{array}$ & \\
\hline & $\begin{array}{l}\text { The use of procalcitonin as a guide to antimicrobial therapy and } \\
\text { relationship to outcome (see Rec 13) }\end{array}$ & \\
\hline & $\begin{array}{l}\text { The determinants of optimal duration of antimicrobial therapy } \\
\text { (see Rec 14) }\end{array}$ & \\
\hline
\end{tabular}

Source control: one pathophysiology Role of source control (see Rec 15 and 16) studies

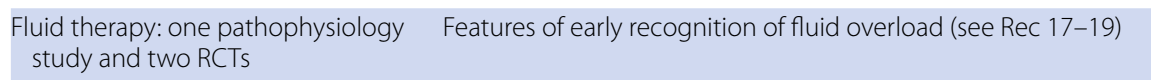

Hemodynamic monitoring: two RCTs

Clinical markers of cardiac output to guide
fluid resuscitation (see Rec 17-19)
Balanced crystalloid vs $0.9 \%$ saline (see Rec
21)
Specific hemodynamic targets ( $>5$ th
vs $>50$ th mean arterial pressure percentile)
(see Rec 24 )
Lactate-guided resuscitation (see Rec 27 )

\begin{tabular}{|c|c|}
\hline \multirow{3}{*}{$\begin{array}{l}\text { Vasoactive medications: three patho- } \\
\text { physiology studies }\end{array}$} & The choice of first-line vasoactive infusion (see Rec 30) \\
\hline & $\begin{array}{l}\text { The optimal threshold for using continuous infusion of vasopres- } \\
\text { sin (see Rec 32) }\end{array}$ \\
\hline & The use and effects of inodilators (see Rec 33) \\
\hline \multirow[t]{3}{*}{$\begin{array}{l}\text { Ventilation: four pathophysiology } \\
\text { studies and five RCTs }\end{array}$} & $\begin{array}{l}\text { Noninvasive modalities to identify the need for early mechanical } \\
\text { ventilation (see Rec 36) }\end{array}$ \\
\hline & $\begin{array}{l}\text { Does early noninvasive mechanical ventilation vs invasive } \\
\text { mechanical ventilation in sepsis-induced PARDS mitigate the } \\
\text { need for subsequent invasive mechanical ventilation (see Rec } \\
\text { 36) }\end{array}$ \\
\hline & $\begin{array}{l}\text { The optimal approach to setting PEEP in mechanical ventilation } \\
\text { for sepsis-induced PARDS (see Rec } 37 \text { ) }\end{array}$ \\
\hline
\end{tabular}

The use of recruitment maneuvers in mechanical ventilation for sepsis-induced PARDS (see Rec 38)
Early-vs delayed-endotracheal intubation for refractory shock without respiratory failure (see Rec 34)

During mechanical ventilation, low-vs moderate-PEEP strategy for sepsis-induced PARDS (see Rec 37)

During mechanical ventilation, prone positioning in sepsis-induced severe PARDS (current RCT will need secondary analysis of this subgroup) (see Rec 39)

High-frequency oscillatory ventilation vs conventional mechanical ventilation in sepsis-induced severe PARDS (current RCT will need secondary analysis of this subgroup) (see Rec 42)

Neuromuscular blocking agent during mechanical ventilation for sepsis-induced severe PARDS (current RCT will need secondary analysis of this subgroup) (see $\operatorname{Rec} 43)$ 
Table 6 (continued)

\begin{tabular}{|c|c|c|}
\hline Subgroup & Pathophysiology & Clinical trials \\
\hline Corticosteroids: one RCT & & $\begin{array}{l}\text { Adjunctive corticosteroids for refractory } \\
\text { septic shock (see Rec 45) }\end{array}$ \\
\hline \multirow[t]{2}{*}{$\begin{array}{l}\text { Endocrine and metabolic therapies: } \\
\text { two pathophysiology studies and } \\
\text { one RCT }\end{array}$} & $\begin{array}{l}\text { The optimal glucose target (between } 140 \text { and } 180 \mathrm{mg} / \mathrm{dL} \text { ) } \\
\text { necessitating control with insulin in children with septic shock } \\
\text { or other sepsis-associated organ dysfunction (see Rec 47) }\end{array}$ & \multirow[t]{2}{*}{$\begin{array}{l}\text { Fever management in children with septic } \\
\text { shock or other sepsis-associated organ } \\
\text { dysfunction (see Rec 50) }\end{array}$} \\
\hline & $\begin{array}{l}\text { Hypocalcemia and supplements in children with septic shock or } \\
\text { other sepsis-associated organ dysfunction (see Rec 48) }\end{array}$ & \\
\hline \multirow{9}{*}{$\begin{array}{l}\text { Nutrition: three pathophysiology } \\
\text { studies and seven RCTs }\end{array}$} & Lipid solution effects on inflammatory physiology (see Rec 54) & \multirow{2}{*}{$\begin{array}{l}\text { Early- vs late- enteral nutrition in children } \\
\text { with septic shock or other sepsis-associated } \\
\text { organ dysfunction (see Recs 51, 52, and 53) }\end{array}$} \\
\hline & $\begin{array}{l}\text { The role of prokinetic agents in immunocompromised children } \\
\text { with septic shock or other sepsis-associated organ dysfunction }\end{array}$ & \\
\hline & & \multirow{2}{*}{$\begin{array}{l}\text { Bolus vs continuous enteral feeding in } \\
\text { children with septic shock or other sepsis- } \\
\text { associated organ dysfunction (see Rec 51, } \\
52 \text {, and 53) }\end{array}$} \\
\hline & \multirow[t]{6}{*}{$\begin{array}{l}\text { The prevalence of low serum vitamin C levels in children with } \\
\text { septic shock or other sepsis-associated organ dysfunction (see } \\
\text { Rec 62) }\end{array}$} & \\
\hline & & $\begin{array}{l}\text { Enteral nutrition vs parenteral supplementa- } \\
\text { tion of nutritional intake in the first } 7 \text { days } \\
\text { of management of children with septic } \\
\text { shock or other sepsis-associated organ } \\
\text { dysfunction (see Rec 53) }\end{array}$ \\
\hline & & $\begin{array}{l}\text { Dietary supplements (selenium, glutamine, } \\
\text { arginine, zinc) in children with septic shock } \\
\text { or other sepsis-associated organ dysfunc- } \\
\text { tion (see Recs 58,59,60, and 61) }\end{array}$ \\
\hline & & $\begin{array}{l}\text { Vitamin C supplementation in children with } \\
\text { septic shock or other sepsis-associated } \\
\text { organ dysfunction (see Rec 62) }\end{array}$ \\
\hline & & $\begin{array}{l}\text { Thiamine deficiency and supplementation in } \\
\text { children with septic shock or other sepsis- } \\
\text { associated organ dysfunction (see Rec 63) }\end{array}$ \\
\hline & & $\begin{array}{l}\text { Vitamin D deficiency and supplementation in } \\
\text { children with septic shock or other sepsis- } \\
\text { associated organ dysfunction (see Rec 64) }\end{array}$ \\
\hline
\end{tabular}

Blood products: two pathophysiol- Optimal hemoglobin level in children with septic shock or other ogy studies sepsis-associated organ dysfunction (see Recs 65 and 66)

The threshold at which the benefits of platelet transfusion outweigh the risks in children with septic shock or other sepsis-associated organ dysfunction (see Rec 67)

\begin{tabular}{|c|c|c|}
\hline \multirow[t]{2}{*}{$\begin{array}{l}\text { Plasma exchange, renal replacement, } \\
\text { and extracorporeal support: two } \\
\text { pathophysiology studies and two } \\
\text { RCTs }\end{array}$} & $\begin{array}{l}\text { Optimal timing and approach for ECMO in refractory shock (see } \\
\text { Rec 74) } \\
\text { To define optimal pre-ECMO candidacy (see Recs } 73 \text { and 74) }\end{array}$ & $\begin{array}{l}\text { Plasma exchange in children with septic } \\
\text { shock or sepsis-associated organ dysfunc- } \\
\text { tion with thrombocytopenia-associated } \\
\text { organ failure (see Rec 70) }\end{array}$ \\
\hline & & $\begin{array}{l}\text { Renal replacement therapy vs diuretics in the } \\
\text { first } 48 \mathrm{~h} \text { in children with septic shock or } \\
\text { other sepsis-associated organ dysfunction } \\
\text { (see Recs 71-72) }\end{array}$ \\
\hline \multicolumn{3}{|l|}{ Immunoglobulins } \\
\hline Prophylaxis: one RCT & & $\begin{array}{l}\text { Stress ulcer prophylaxis in relation to feed- } \\
\text { ing in children with septic shock or other } \\
\text { sepsis-associated organ dysfunction (see } \\
\text { Rec 76) }\end{array}$ \\
\hline
\end{tabular}

ECMO extracorporeal membrane oxygenation, PARDS pediatric acute respiratory distress syndrome, PEEP positive end-expiratory pressure, Q/ quality improvement, $R C T$ randomized clinical trial, $R e c$ recommendation number

studies at different phases of management (i.e., recognition, fluid resuscitation, first $48 \mathrm{~h}$ ).

\section{Author details}

${ }^{1}$ Children's Hospital of Philadelphia, University of Pennsylvania Perelman School of Medicine, Philadelphia, PA, USA. ${ }^{2}$ Great Ormond Street Hospital for Children, London, UK. ${ }^{3}$ Department of Medicine, Division of Critical Care, McMaster University, Hamilton, ON, Canada. ${ }^{4}$ Department of Health Research Methods and Impact, McMaster University, Hamilton, ON, Canada. ${ }^{5}$ Department of Pediatrics, Boston Children's Hospital and Harvard Medical School, Boston, MA, USA. ${ }^{6}$ C.S. Mott Children's Hospital, Ann Arbor, MI, USA. ${ }^{7}$ St. Mary's Hospital, London, UK. ${ }^{8}$ Paediatric Critical Care Research Group, The University 
of Queensland and Queensland Children's Hospital, Brisbane, QLD, Australia. ${ }^{9}$ Red Cross War Memorial Children's Hospital and University of Cape Town, Cape Town, South Africa. ${ }^{10}$ Children's Hospital of Pittsburgh, Pittsburgh, PA, USA. ${ }^{11}$ University of Liverpool, Liverpool, UK. ${ }^{12}$ Connecticut Children's Medical Center, Hartford, CT, USA. ${ }^{13}$ Duke Children's, Durham, NC, USA. ${ }^{14}$ St. Christopher's Hospital for Children, Philadelphia, PA, USA. ${ }^{15}$ Texas Children's Hospital, Houston, TX, USA. ${ }^{16}$ Paris South University Hospitals-Assistance Publique Hopitaux de Paris, Paris, France. ${ }^{17}$ Physiopathology and Therapeutic Innovation Unit-INSERM U999, South Paris-Saclay University, Paris, France. ${ }^{18}$ King's College Hospital, London, UK. ${ }^{19}$ University Hospital Southampton NHS Foundation Trust and University of Southampton, Southampton, UK. ${ }^{20}$ The Latin America Sepsis Institute, São Paulo, Brazil. ${ }^{21}$ Nationwide Children's Hospital, Columbus, OH, USA. ${ }^{22}$ Rady Children's Hospital, San Diego, CA, USA. ${ }^{23}$ Centre Hospitalier Universitaire de Lyon, Lyon, France. ${ }^{24}$ Erasmus University Medical Center, Rotterdam, The Netherlands. ${ }^{25}$ All India Institute of Medical Sciences, New Delhi, India. ${ }^{26}$ Children's Hospital of Richmond at VCU, Richmond, VA, USA. ${ }^{27}$ Beatrix Children's Hospital, Groningen, The Netherlands. ${ }^{28}$ Radboud University Medical Centre, Nijmegen, The Netherlands. ${ }^{29}$ National University Health System, Singapore, Singapore. ${ }^{30}$ Royal Children's Hospital, Melbourne, VIC, Australia. ${ }^{31}$ Department of Anesthesiology, Critical Care and Pain, Boston Children's Hospital and Harvard Medical School, Boston, MA, USA. ${ }^{32}$ Rigshospitalet Hospital, Copenhagen, Denmark. ${ }^{33}$ Children's Hospital of Los Angeles, Los Angeles, CA, USA. ${ }^{34}$ New York University Langone Medical Center, New York, NY, USA. ${ }^{35}$ Stony Brook University, Stony Brook, NY, USA. ${ }^{36}$ Advocate Children's Hospital, Park Ridge, IL, USA. ${ }^{37}$ Apollo Hospitals, Chennai, India. 38 Johns Hopkins Children's Center, Baltimore, MD, USA. ${ }^{39}$ Children's Hospital Colorado, Aurora, CO, USA. ${ }^{40}$ University of the West of England, Bristol, UK. ${ }^{41}$ College of Nursing, University of lowa, lowa City, IA, USA. ${ }^{42}$ St. Jude Children's Research Hospital, Memphis, TN, USA. ${ }^{43}$ Cincinnati Children's Hospital, Cincinnati, OH, USA. ${ }^{44}$ Seattle Children's Hospital, Seattle, WA, USA. ${ }^{45}$ British Columbia Children's Hospital, Vancouver, BC, Canada. ${ }^{46}$ Institute of Integrative Biology of the Cell-CNRS, CEA, Univ Paris Sud, Gif-Sur-Yvette, France.

\section{Acknowledgements}

We wish to thank Darlene Barkman, MA, and Janna Pogers, PT, MPT, NCS, CSRS for their sensitive and insightful comments from the perspective of parents of children with sepsis. Their input, particularly related to ranking the importance of outcomes to consider through the literature search, provided valuable direction to the panel. We also wish to thank Rebecca Skidmore and James D. Medd for their dedication as they conducted the literature searches for the five panels. Their experience and professionalism contributed greatly to the final publication. Finally, appreciation is extended to Deborah L. McBride for project management and editorial support.

The following sponsoring organizations with formal liaison appointees endorse this guideline: American Academy of Pediatrics; Australia and New Zealand Intensive Care Society; Canadian Critical Care Society; European Society of Intensive Care Medicine; European Society of Paediatric and Neonatal Intensive Care; Pediatric Infectious Disease Society; Scandinavian Society of Anaesthesiology and Intensive Care Medicine; Society of Critical Care Medicine; UK Sepsis Trust; World Federation of Pediatric Intensive and Critical Care Societies. These guidelines were solely funded by the European Society of Intensive Care Medicine and the Society of Critical Care Medicine.

\section{Compliance with ethical standards}

\section{Conflict of interest}

Drs. Weiss and Peters served as arbiters for conflict interest management and adjudication throughout the guidelines process following standard operating procedures set forth by Society of Critical Care Medicine (SCCM) and endorsed by European Society of Intensive Care Medicine. Dr. Weiss participates in Pediatric Acute Lung Injury and Sepsis Investigators (PALISI) and Shock Society. Dr. Peters participates in the UK PICS study group (vice-chair) and has testified as an expert witness in cases of clinical negligence, causation of injuries. Dr. Agus participates in the American Academy of Pediatrics (AAP), Pediatric Academic Societies (PAS), American Pediatric Society, Society for Pediatric Research, and The American Society for Clinical Investigation, and he has testified as an expert witness in cases related to ICU and/or endocrinology in children. Dr. Flori participates in American Thoracic Society (ATS) State Chapter (Executive Board Member-Michigan and California State Chapters) and PALISI Network, Grant funding from Gerber Foundation (Steering committee member for various studies being implemented through the Network from intramural funding, governmental or other Foundation Grant funding). Dr. Nadel received funding form La Jolla Pharmaceutical (consulting), and he participates in the European Society of Pediatric and Neonatal Intensive Care (ESPNIC) (Medical President). Dr. Brierley participates in the ESPNIC. Dr. Carrol participates in National Institute for Health and Care Excellence (NICE) (Diagnostic Advisory Committee panel) and National Institutes for Health Research (two scientific panels, i4i and DTF). Dr. Cheifetz participates in American Association for Respiratory Care and ATS (volunteer activities) and has testified as an expert witness for medical malpractice cases, he is an advisor to Philips, and a contributor to Up-to-Date. Dr. Cies received funding from Allergan, Merck, Thermo Fisher Scientific, and Atlantic Diagnostic Laboratories (consultant), and he participates in Pediatric Pharmacy Advocacy Group (multiple positions), Society of Infectious Diseases Pharmacists (Vice-Chair of the Inter-organizations Liaison Committee), and the American College of Clinical Pharmacists (member and fellow). Dr. Cruz has testified as an expert witness in cases of children with tuberculosis-related meningitis and is an associate editor for Pediatrics. Dr. De Luca serves as Medical President-elect on the Executive Committee of ESPNIC, he served as a consultant and lecturer on the external advisory board and received research and educational grants from Chiesi Farmaceutici S.p.A and AbbVie, and travel grants from AbbVie, he has been a lecturer for Philips, Radiometer, and Waire. Dr. Faust served as chair of the UK NICE Guideline Committee for Sepsis in Children and Adults published in 2016 and for Lyme disease published in 2018, serves as a regional representative to the UK NHS England Clinical Reference Group for commissioning pediatric specialist medicine care (immunology and infection). Dr. Hall receives funding from La Jolla Pharmaceuticals (consultant on the data safety monitoring board for a clinical trial of a sepsis therapeutic), and he participates in the ATS (online journal club editor) and the American Board of Pediatrics (Critical Care Medicine sub-board). Dr. Ishimine participates in SAEM (Consensus Conference Co-Chair), American Board of Pediatrics/American Board of Emergency Medicine (Immediate Past Chair of the Pediatric Emergency Medicine Subboard), and the American College of Emergency Physicians (Pediatric Emergency Medicine Committee member). Dr. Javouhey received funding from CSL Behring (trial on IV Immunoglobulins in toxic shock syndrome in children). Dr. Karam participates in BloodNet, PALISI, ISBT, AABB, and CCCTG. Dr. Kneyber participates in the European Society for Pediatric and Neonatal Intensive Care. Dr. MacLaren participates in Extracorporeal Life Support Organization (Executive Committee). Dr. Mehta participates in the American Society for Parenteral and Enteral Nutrition (president). Dr. Møller participates in the Science Systems and Applications, Inc (board member). Dr. Newth received funding from Philips Research North America (consulting concerning monitoring in PICU), and he participates in the ATS. Dr. Nishisaki's institutional department receives an unrestricted grant from Nihon Kohden (involves an activity to develop a device to measure capillary refill time), and he participates in the Society for Simulation in Healthcare and International Society for Pediatric Simulation. Dr. Nunnally participates in American College of Critical Care Medicine (Regent), Society of Critical Care Anesthesiologists (director), American Society of Anesthesiologists (committee), International Anesthesia Research Society, and NYSA. Dr. Randolph's institution received funding from Genentech (influenza biomarker study research support); she has received funding from Bristol Myers Squibb (consultant in 2017) and La Jolla Pharmaceuticals (design of pediatric septic shock trial of angiotensin II); and she participates in the ATS and the International Sepsis Forum. Dr. Ranjit participates as the Chancellor of College of Pediatric Critical Care, India. Dr. Tume participates in ESPNIC (Nursing President) and the UK PICS Scientific and Education Committee. Dr. Verger participates in the American Association of Critical-Care Nurses (Cert. Corp. Governance Committee) and the Academy of Nursing (Acute and Critical Care Special Interest Group). Dr. Williams participates in the Pediatric Cardiac Intensive Care Society. $\mathrm{Dr}$. Wolf received funding support for participation in industry-sponsored research from Merck \& Co, Astellas, and Cempra Pharmaceuticals, and he received other support from Karius, Empatica, and Bluespark Technologies. Dr. Zimmerman (Past President of SCCM) received funding from Immunexpress, Seattle (sepsis biomarker research), and he participates in the AAP and PAS. Dr. Tissieres received funding from Baxter acute therapies, Bristol-Myers Squibb Company, Chiesi Farmaceutici S.p.A., Faron Pharmaceuticals (consulting, renal replacement therapy), and Biomerieux, funding from La Jolla Pharmaceuticals, Chiesi Farmaceutici S.p.A., and is President ESPNIC (research grant, biomarkers sepsis), and he participates in the Swiss Intensive Care Society, Swiss Pediatric Society, and the French Society of Intensive Care. The remaining authors have disclosed that they do not have any potential conflicts of interest. 


\section{Appendix: Summary of guidelines}

\section{Screening, diagnosis, and systematic management of sepsis}

1. In children who present as acutely unwell, we suggest implementing systematic screening for timely recognition of septic shock and other sepsis-associated organ dysfunction (weak recommendation, very low quality of evidence). PICO 1

Remarks: Systematic screening needs to be tailored to the type of patients, resources, and procedures within each institution. Evaluation for the effectiveness and sustainability of screening should be incorporated as part of this process

2. We were unable to issue a recommendation about using blood lactate values to stratify children with suspected septic shock or other sepsis-associated organ dysfunction into low-versus high-risk of having septic shock or sepsis. PICO 2

3. We recommend implementing a protocol/guideline for management of children with septic shock or other sepsis-associated organ dysfunction (BPS). PICO 3

4. We recommend obtaining blood cultures before initiating antimicrobial therapy in situations where this does not substantially delay antimicrobial administration (BPS). PICO 4

\section{Antimicrobial therapy}

5. In children with septic shock, we recommend starting antimicrobial therapy as soon as possible, within $1 \mathrm{~h}$ of recognition (strong recommendation, very low quality of evidence). PICO 6

6. In children with sepsis-associated organ dysfunction but without shock, we suggest starting antimicrobial therapy as soon as possible after appropriate evaluation, within $3 \mathrm{~h}$ of recognition (weak recommendation, very low quality of evidence). PICO 6

7. We recommend empiric broad-spectrum therapy with one or more antimicrobials to cover all likely pathogens (BPS). PICO 5

8. Once the pathogen(s) and sensitivities are available, we recommend narrowing empiric antimicrobial therapy coverage (BPS). PICO 5

9. If no pathogen is identified, we recommend narrowing or stopping empiric antimicrobial therapy according to clinical presentation, site of infection, host risk factors, and adequacy of clinical improvement in discussion with infectious disease and/or microbiological expert advice (BPS). PICO 5

10. In children without immune compromise and without high risk for multidrug-resistant pathogens, we suggest against the routine use of empiric multiple antimicrobials directed against the same pathogen for the purpose of synergy (weak recommendation, very low quality of evidence). PICO 8/9

Remarks: In certain situations, such as confirmed or strongly suspected group B streptococcal sepsis, use of empiric multiple antimicrobials directed against the same pathogen for the purpose of synergy may be indicated

11. In children with immune compromise and/or at high risk for multidrug-resistant pathogens, we suggest using empiric multi-drug therapy when septic shock or other sepsis-associated organ dysfunction is present/suspected (weak recommendation, very low quality of evidence). PICO 8/9

12. We recommend using antimicrobial dosing strategies that have been optimized based on published pharmacokinetic/pharmacodynamic principles and with consideration of specific drug properties (BPS). $\mathrm{PICO} 7$

13. In children with septic shock or sepsis-associated organ dysfunction who are receiving antimicrobials, we recommend daily assessment (e.g., clinical, laboratory assessment) for de-escalation of antimicrobial therapy (BPS). PICO 11

Remarks: This assessment should include a review of the ongoing indication for empiric antimicrobial therapy after the first $48 \mathrm{~h}$ that is guided by microbiologic results and in response to clinical improvement and/or evidence of infection resolution. This recommendation applies to patients being treated with empiric, targeted, and combination therapy
14. We recommend determining the duration of antimicrobial therapy according to the site of infection, microbial etiology, response to treatment, and ability to achieve source control (BPS). PICO 10

\section{Source control}

15. We recommend that emergent source control intervention be implemented as soon possible after a diagnosis of an infection amenable to a source control procedure is made (BPS). PICO 12 Remarks: Appropriate diagnostic testing to identify the site of infection and microbial etiology should be performed, and advice from specialist teams (e.g., infectious diseases, surgery) should be sought, as appropriate, in order to prioritize interventions needed to achieve source control

16. We recommend removal of intravascular access devices that are confirmed to be the source of sepsis or septic shock after other vascular access has been established and depending on the pathogen and the risks/benefits of a surgical procedure (strong recommendation, low quality of evidence). PICO 13

\section{Fluid therapy}

17. In healthcare systems with availability of intensive care, we suggest administering up to $40-60 \mathrm{~mL} / \mathrm{kg}$ in bolus fluid $(10-20 \mathrm{~mL} / \mathrm{kg}$ per bolus) over the first hour, titrated to clinical markers of cardiac output and discontinued if signs of fluid overload develop, for the initial resuscitation of children with septic shock or other sepsis-associated organ dysfunction (weak recommendation, low quality of evidence). PICO 17

18. In healthcare systems with no availability of intensive care and in the absence of hypotension, we recommend against bolus fluid administration while starting maintenance fluids (strong recommendation, high quality of evidence). PICO 17

19. In healthcare systems with no availability of intensive care, if hypotension is present, we suggest administering up to $40 \mathrm{~mL} / \mathrm{kg}$ in bolus fluid (10-20 mL/kg per bolus) over the first hour with titration to clinical markers of cardiac output and discontinued if signs of fluid overload develop (weak recommendation, low quality of evidence). PICO 17 Remarks: Clinical markers of cardiac output may include heart rate, blood pressure, capillary refill time, level of consciousness, and urine output. In all settings, the need for fluid administration should be guided by frequent reassessment of clinical markers of cardiac output, serial blood lactate measurement and advanced monitoring, when available. Signs of fluid overload that should limit further fluid bolus therapy may include clinical signs of pulmonary edema or new or worsening hepatomegaly

20. We suggest using crystalloids, rather than albumin, for the initial resuscitation of children with septic shock or other sepsis-associated organ dysfunction (weak recommendation, moderate quality of evidence). PICO 15 Remarks: Although there is no difference in outcomes, this recommendation takes into consideration cost and other barriers of administering albumin compared to crystalloids

21. We suggest using balanced/buffered crystalloids, rather than $0.9 \%$ saline, for the initial resuscitation of children with septic shock or other sepsis-associated organ dysfunction (weak recommendation, very low quality of evidence). PICO 14

22. We recommend against using starches in the acute resuscitation of children with septic shock or other sepsis-associated organ dysfunction (strong recommendation, moderate quality of evidence) PICO 16

23. We suggest against using gelatin in the resuscitation of children with septic shock or other sepsis-associated organ dysfunction (weak recommendation, low quality of evidence). PICO 16

\section{Hemodynamic monitoring}

24. We were unable to issue a recommendation about whether to target mean arterial blood pressure (MAP) at the $5^{\text {th }}$ or $50^{\text {th }}$ percentile for age in children with septic shock and other sepsis-associated organ dysfunction. PICO 21 
25. We suggest not using bedside clinical signs in isolation to categorize septic shock in children as "warm" or "cold" (weak recommendation, very low quality of evidence). PICO 20

26. We suggest using advanced hemodynamic variables, when available, in addition to bedside clinical variables to guide the resuscitation of children with septic shock or other sepsis-associated organ dysfunction (weak recommendation, low quality of evidence). PICO 18 Remarks: Advanced hemodynamic monitoring may include cardiac output/cardiac index, systemic vascular resistance, or central venous oxygen saturation $\left(\mathrm{SCVO}_{2}\right)$

27. We suggest using trends in blood lactate levels, in addition to clinical assessment, to guide resuscitation of children with septic shock and other sepsis-associated organ dysfunction (weak recommendation, very low quality of evidence). PICO 19

Remarks: In children with an elevated blood lactate, repeat testing that reveals a persistent elevation in blood lactate may indicate incomplete hemodynamic resuscitation and should prompt efforts, as needed, to further promote hemodynamic stability

\section{Vasoactive medications}

28. We suggest using epinephrine, rather than dopamine, in children with septic shock (weak recommendation, low quality of evidence). PICO 22

29. We suggest using norepinephrine, rather than dopamine, in children with septic shock (weak recommendation, very low quality of evidence). PICO 23

30. We were unable to issue a recommendation for a specific first-line vasoactive infusion for children with septic shock. PICO 22/23

31. We were unable to issue a recommendation about initiating vasoactive agents through peripheral access in children with septic shock. PICO 26

Remarks: It is reasonable to begin vasoactive infusions after $40-60 \mathrm{~mL} / \mathrm{kg}$ of fluid resuscitation if the patient continues to have evidence of abnormal perfusion. Either epinephrine or norepinephrine may be administered through a peripheral vein (or intraosseous, if in place) if central venous access is not readily accessible. Dopamine may be substituted as the first-line vasoactive infusion, administered either peripherally or centrally, if epinephrine or norepinephrine is not readily available

32. We suggest either adding vasopressin or further titrating catecholamines in children with septic shock who require high-dose catecholamines (weak recommendation, low quality of evidence). PICO 25 Remarks: No consensus was achieved on the optimal threshold for initiating vasopressin. Therefore, this decision should be made according to individual clinician preference

33. We were unable to issue a recommendation about adding an inodilator in children with septic shock and cardiac dysfunction despite other vasoactive agents. PICO 24

\section{Ventilation}

34. We were unable to issue a recommendation about whether to intubate children with fluid-refractory, catecholamine-resistant septic shock. PICO 27

35. We suggest not to use etomidate when intubating children with septic shock or other sepsis-associated organ dysfunction (weak recommendation, low quality of evidence). PICO 28

36. We suggest a trial of non-invasive mechanical ventilation (over invasive mechanical ventilation) in children with sepsis-induced pediatric ARDS (PARDS) without a clear indication for intubation and who are responding to initial resuscitation (weak recommendation, very low quality of evidence). PICO 29

Remarks: When non-invasive mechanical ventilation is initiated, clinicians should carefully and frequently re-evaluate the patient's condition
37. We suggest using high positive end-expiratory pressure (PEEP) in children with sepsis-induced PARDS (weak recommendation, very low quality of evidence). PICO 30

Remarks: The exact level of high PEEP has not been tested or determined in PARDS patients. Some RCTs and observational studies in PARDS have used and advocated for use of the ARDS-network PEEP to fractional inspired oxygen $\left(\mathrm{FiO}_{2}\right)$ grid though adverse hemodynamic effects of high PEEP may be more prominent in children with septic shock

38. We cannot suggest for or against the use of recruitment maneuvers in children with sepsis-induced PARDS and refractory hypoxemia. PICO 31

Remarks: If a recruitment maneuver is considered, the use of a stepwise, incremental and decremental PEEP titration maneuver is preferred over sustained inflation techniques that have not been optimized through direct testing in PARDS patients. All PARDS patients must be carefully monitored for tolerance of the maneuver

39. We suggest a trial of prone positioning in children with sepsis and severe PARDS (weak recommendation, low quality of evidence). PICO 32

Remarks: Research trials in adults with ARDS and children with PARDS have emphasized prone positioning for at least $12 \mathrm{~h}$ per day, as tolerated

40. We recommend against the routine use of inhaled nitric oxide (iNO) in all children with sepsis-induced PARDS (strong recommendation, low quality of evidence). PICO 33

41. We suggest using iNO as a rescue therapy in children with sepsisinduced PARDS and refractory hypoxemia after other oxygenation strategies have been optimized (weak recommendation, moderate quality of evidence). PICO 33

42. We were unable to issue a recommendation to use high-frequency oscillatory ventilation (HFOV) versus conventional ventilation in children with sepsis-induced PARDS. PICO 34

43. We suggest using neuromuscular blockade in children with sepsis and severe PARDS (weak recommendation, very low quality of evidence). PICO 35

Remarks: The exact duration of neuromuscular blockade use in severe PARDS patients has not been determined to date. Most of the adult RCT data and pediatric observational data support treatment for 24-48 $\mathrm{h}$ after ARDS onset

\section{Corticosteroids}

44. We suggest against using intravenous hydrocortisone to treat children with septic shock if adequate fluid resuscitation and vasopressor therapy are able to restore hemodynamic stability (weak recommendation, low quality of evidence). PICO 47

45. We suggest that either intravenous hydrocortisone or no hydrocortisone may be used if adequate fluid resuscitation and vasopressor therapy are not able to restore hemodynamic stability (weak recommendation, low quality of evidence). PICO 47

\section{Endocrine and metabolic}

46. We recommend against insulin therapy to maintain glucose target at or below $140 \mathrm{mg} / \mathrm{dL}$ ( $7.8 \mathrm{mmol} / \mathrm{L}$ ) (strong recommendation, moderate quality of evidence). PICO 52/60

47. We were unable to issue a recommendation regarding what blood glucose range to target for children with septic shock and other sepsis-associated organ dysfunction. PICO 52/60

48. We were unable to issue a recommendation as to whether to target normal blood calcium levels in children with septic shock or sepsisassociated organ dysfunction. PICO 62

49. We suggest against the routine use of levothyroxine in children with septic shock and other sepsis-associated organ dysfunction in a sick euthyroid state (weak recommendation, low quality of evidence). PICO 63 
50. We suggest either antipyretic therapy or a permissive approach to fever in children with septic shock or other sepsis-associated organ dysfunction (weak recommendation, moderate quality of evidence). $\mathrm{PICO} 64$

\section{Nutrition}

51. We were unable to issue a recommendation regarding early hypocaloric/trophic enteral feeding followed by slow increase to full enteral feeding versus early full enteral feeding in children with septic shock or sepsis-associated organ dysfunction without contraindications to enteral feeding. PICO 51

52. We suggest not withholding enteral feeding solely on the basis of vasoactive-inotropic medication administration (weak recommendation, low quality of evidence). PICO 48

Remarks: Enteral feeding is not contraindicated in children with septic shock after adequate hemodynamic resuscitation who no longer require escalating doses of vasoactive agents or in whom weaning of vasoactive agents has started

53. We suggest enteral nutrition as the preferred method of feeding and that parenteral nutrition may be withheld in the first 7 days of PICU admission in children with septic shock or other sepsis-associated organ dysfunction (weak recommendation, moderate quality of evidence). PICO 49/50

54. We suggest against supplementation with specialized lipid emulsions in children with septic shock or other sepsis-associated organ dysfunction (weak recommendation, very low quality of evidence). PICO 53

55. We suggest against the routine measurements of gastric residual volumes (GRV) in children with septic shock or other sepsis-associated organ dysfunction (weak recommendation, low quality of evidence). PICO 54

56. We suggest administering enteral feeds through a gastric tube, rather than a post-pyloric feeding tube, to children with septic shock or other sepsis-associated organ dysfunction who have no contraindications to enteral feeding (weak recommendation, low quality of evidence). PICO 55

57. We suggest against the routine use of prokinetic agents for the treatment of feeding intolerance in children with septic shock or other sepsis-associated organ dysfunction (weak recommendation, low quality of evidence). PICO 56

58. We suggest against the use of selenium in children with septic shock or other sepsis-associated organ dysfunction (weak recommendation, low quality of evidence). PICO 57

59. We suggest against the use of glutamine supplementation in children with septic shock or other sepsis-associated organ dysfunction (weak recommendation, low quality of evidence). PICO 58

60. We suggest against the use of arginine in the treatment of children with septic shock or other sepsis-associated organ dysfunction (weak recommendation, very low quality of evidence). PICO 59

61. We suggest against using zinc supplementation in children with septic shock and other sepsis-associated organ dysfunction (weak recommendation, very low quality of evidence). PICO 61

62. We suggest against the use of ascorbic acid (vitamin C) in the treatment of children with septic shock or other sepsis-associated organ dysfunction (weak recommendation, very low quality of evidence). PICO 65

63. We suggest against the use of thiamine to treat children with sepsisassociated organ dysfunction (weak recommendation, low quality of evidence). PICO 66
64. We suggest against the acute repletion of vitamin D deficiency

(VDD) for treatment of septic shock or other sepsis-associated organ dysfunction (weak recommendation, very low quality of evidence).

PICO 67

\section{Blood products}

65. We suggest against transfusion of red blood cells if the blood hemoglobin concentration is $\geq 7 \mathrm{~g} / \mathrm{dL}$ in hemodynamically stabilized children with septic shock or other sepsis-associated organ dysfunction (weak recommendation, low quality of evidence). PICO 38 Remarks: According to the 2018 Transfusion and Anemia Expertise Initiative (TAXI) guidelines, for the purposes of red blood cell transfusion, "hemodynamically stabilized" is defined as a mean arterial blood pressure higher than 2 standard deviations below normal for age and no increase in vasoactive medications for at least $2 \mathrm{~h}$

66. We cannot make a recommendation regarding hemoglobin transfusion thresholds for critically ill children with unstable septic shock. $\mathrm{PICO} 38$

67. We suggest against prophylactic platelet transfusion based solely on platelet levels in non-bleeding children with septic shock or other sepsis-associated organ dysfunction and thrombocytopenia (weak recommendation, very low quality of evidence). PICO 40

68. We suggest against prophylactic plasma transfusion in non-bleeding children with septic shock or other sepsis-associated organ dysfunction and coagulation abnormalities (weak recommendation, very low quality of evidence). PICO 39

Remarks: Prophylactic plasma transfusion refers to situations in which there is an abnormality in laboratory coagulation testing but no active bleeding

\section{Plasma exchange, renal replacement, and extracorporeal support}

69. We suggest against using plasma exchange in children with septic shock or other sepsis-associated organ dysfunction without thrombocytopenia-associated multiple organ failure (TAMOF) (weak recommendation, very low quality of evidence). PICO 37

70. We cannot suggest for or against the use of plasma exchange in children with septic shock or other-sepsis-associated organ dysfunction with TAMOF. PICO 37

71. We suggest using renal replacement therapy to prevent or treat fluid overload in children with septic shock or other sepsis-associated organ dysfunction who are unresponsive to fluid restriction and diuretic therapy (weak recommendation, very low quality of evidence). $\mathrm{PICO} 43$

72. We suggest against high-volume hemofiltration over standard hemofiltration in children with septic shock or other sepsis-associated organ dysfunction who are treated with renal replacement therapy (weak recommendation, low quality of evidence). PICO 44

73. We suggest using veno-venous (VV) extracorporeal membrane oxygenation (ECMO) in children with sepsis-induced PARDS and refractory hypoxia (weak recommendation, very low quality of evidence). $\mathrm{PICO} 36$

74. We suggest using veno-arterial (VA) ECMO as a rescue therapy in children with septic shock only if refractory to all other treatments (weak recommendation, very low quality of evidence). PICO 45

\section{Immunoglobulins}

75. We suggest against the routine use of intravenous immune globulin (IVIG) in children with septic shock or other sepsis-associated organ dysfunction (weak recommendation, low quality of evidence). PICO 46

Remarks: Although routine use of IVIG is not recommended, select patients may benefit from such treatment 


\section{Prophylaxis}

76. We suggest against the routine use of stress ulcer prophylaxis in critically ill children with septic shock or other sepsis-associated organ dysfunction, except for high-risk patients (weak recommendation, very low quality of evidence). PICO 41

Remarks: Although routine stress-ulcer prophylaxis is not recommended, some high-risk patients may benefit from stress ulcer prophylaxis. Studies have supported benefit of stress ulcer prophylaxis when baseline rate of clinically important bleeding is approximately $13 \%$

77. We suggest against routine deep vein thrombosis (DVT) prophylaxis (mechanical or pharmacologic) in critically ill children with septic shock or other sepsis-associated organ dysfunction, but potential benefits may outweigh risks and costs in specific populations (weak recommendation, low quality of evidence). PICO 42

\section{Publisher's Note}

Springer Nature remains neutral with regard to jurisdictional claims in published maps and institutional affiliations.

\section{Published online: 7 February 2020}

\section{References}

1. Fleischmann-Struzek C, Goldfarb DM, Schlattmann P et al (2018) The global burden of paediatric and neonatal sepsis: a systematic review. Lancet Respir Med 6:223-230

2. Balamuth F, Weiss SL, Neuman Ml et al (2014) Pediatric severe sepsis in U.S. children's hospitals. Pediatr Crit Care Med 15:798-805

3. Odetola FO, Gebremariam A, Freed GL (2007) Patient and hospital correlates of clinical outcomes and resource utilization in severe pediatric sepsis. Pediatrics 119:487-494

4. Ruth A, McCracken CE, Fortenberry JD et al (2014) Pediatric severe sepsis: current trends and outcomes from the Pediatric Health Information Systems database. Pediatr Crit Care Med 15:828-838

5. Weiss SL, Fitzgerald JC, Pappachan J, Sepsis Prevalence, Outcomes, and Therapies (SPROUT) Study Investigators and Pediatric Acute Lung Injury and Sepsis Investigators (PALISI) Network et al (2015) Global epidemiology of pediatric severe sepsis: The sepsis prevalence, outcomes, and therapies study. Am J Respir Crit Care Med 191:1147-1157

6. Schlapbach LJ, Straney L, Alexander J, ANZICS Paediatric Study Group et al (2015) mortality related to invasive infections, sepsis, and septic shock in critically ill children in Australia and New Zealand, 2002-13: a multicentre retrospective cohort study. Lancet Infect Dis 15:46-54

7. Ames SG, Davis BS, Angus DC et al (2018) Hospital variation in riskadjusted pediatric sepsis mortality. Pediatr Crit Care Med 19:390-396

8. Evans IVR, Phillips GS, Alpern ER et al (2018) Association between the New York sepsis care mandate and in-hospital mortality for pediatric sepsis. JAMA 320:358-367

9. Prout AJ, Talisa VB, Carcillo JA et al (2018) Children with chronic disease bear the highest burden of pediatric sepsis. J Pediatr 199(194-199):e1

10. Morin L, Ray S, Wilson C, ESPNIC Refractory Septic Shock Definition Taskforce the Infection Systemic Inflammation Sepsis section of ESPNIC et al (2016) Refractory septic shock in children: a European Society of Paediatric and Neonatal Intensive Care definition. Intensive Care Med 42:1948-1957

11. Schlapbach L, MacLaren G, Festa M, Australian \& New Zealand Intensive Care Society (ANZICS) Centre for Outcomes \& Resource Evaluation (CORE) and Australian \& New Zealand Intensive Care Society (ANZICS) Paediatric Study Group et al (2017) Prediction of pediatric sepsis mortality within $1 \mathrm{~h}$ of intensive care admission. Intensive Care Med 43:1085-1096

12. Weiss SL, Balamuth F, Hensley J et al (2017) The epidemiology of hospital death following pediatric severe sepsis: when, why, and how children with sepsis die. Pediatr Crit Care Med 18:823-830
13. Cvetkovic M, Lutman D, Ramnarayan P et al (2015) Timing of death in children referred for intensive care with severe sepsis: implications for interventional studies. Pediatr Crit Care Med 16:410-417

14. World Health Organization: Guideline (2016) Updates on paediatric emergency triage, assessment and treatment: care of critically-III children. Geneva, Switzerland, World Health Organization. https://www. ncbi.nlm.nih.gov/books/NBK350523/. Accessed 18 Aug 2019

15. Davis AL, Carcillo JA, Aneja RK et al (2017) The American college of critical care medicine clinical practice parameters for hemodynamic support of pediatric and neonatal septic shock: executive summary. Pediatr Crit Care Med 18:884-890

16. NICE. National Institute for Health and Care Excellence (2016) Sepsis: recognition, diagnosis and early Management (NICE Guideline 51). https://www.nice.org.uk/guidance/ng51. Accessed 18 Aug 2019

17. Goldstein B, Giroir B, Randolph A, International Consensus Conference on Pediatric Sepsis (2005) International pediatric sepsis consensus conference: definitions for sepsis and organ dysfunction in pediatrics. Pediatr Crit Care Med 6:2-8

18. Singer M, Deutschman CS, Seymour CW et al (2016) The third international consensus definitions for sepsis and septic shock (Sepsis-3). JAMA 315:801-810

19. Matics TJ, Sanchez-Pinto LN (2017) Adaptation and validation of a pediatric sequential organ failure assessment score and evaluation of the sepsis-3 definitions in critically ill children. JAMA Pediatr 171:e172352

20. Schlapbach LJ, Straney L, Bellomo R et al (2018) Prognostic accuracy of age-adapted SOFA, SIRS, PELOD-2, and qSOFA for in-hospital mortality among children with suspected infection admitted to the intensive care unit. Intensive Care Med 44:179-188

21. Schlapbach LJ, Kissoon N (2018) Defining pediatric sepsis. JAMA Pediatr 172:312-314

22. Leteurtre S, Duhamel A, Salleron J, Groupe Francophone de Réanimation et d'Urgences Pédiatriques (GFRUP) et al (2013) PELOD-2: an update of the PEdiatric logistic organ dysfunction score. Crit Care Med 41:1761-1773

23. Proulx F, Gauthier M, Nadeau D et al (1994) Timing and predictors of death in pediatric patients with multiple organ system failure. Crit Care Med 22:1025-1031

24. Pediatric Acute Lung Injury Consensus Conference Group (2015) Pediatric acute respiratory distress syndrome: consensus recommendations from the pediatric acute lung injury consensus conference. Pediatr Crit Care Med 16:428-439

25. Higgins JP, Altman DG, Gøtzsche PC, Cochrane Bias Methods Group; Cochrane Statistical Methods Group et al (2011) The cochrane collaboration's tool for assessing risk of bias in randomised trials. BMJ 343:d5928

26. Wells G, Shea B, O'Connell D et al (2009) The Newcastle-Ottawa Scale (NOS) for assessing the quality of nonrandomised studies in meta-analyses. http://www.ohri.ca/programs/clinical_epidemiology/oxford.htm. Accessed 18 Aug 2019

27. Guyatt GH, Oxman AD, Vist GE, GRADE Working Group: GRADE et al (2008) An emerging consensus on rating quality of evidence and strength of recommendations. BMJ 336:924-926

28. Neumann I, Brignardello-Petersen R, Wiercioch W et al (2016) The GRADE evidence-to-decision framework: a report of its testing and application in 15 international guideline panels. Implement Sci 11:93

29. Alexander PE, Gionfriddo MR, Li SA et al (2016) A number of factors explain why WHO guideline developers make strong recommendations inconsistent with GRADE guidance. J Clin Epidemiol 70:111-122

30. Guyatt GH, Schünemann HJ, Djulbegovic B et al (2015) Guideline panels should not GRADE good practice statements. J Clin Epidemiol 68:597-600

31. Paul R, Melendez E, Stack A et al (2014) Improving adherence to PALS septic shock guidelines. Pediatrics 133:e1358-e1366

32. Paul R, Neuman MI, Monuteaux MC et al (2012) Adherence to PALS sepsis guidelines and hospital length of stay. Pediatrics 130:e273-e280

33. Lane RD, Funai $T$, Reeder $R$ et al (2016) High reliability pediatric septic shock quality improvement initiative and decreasing mortality. Pediatrics 138:e20154153

34. Balamuth F, Weiss SL, Fitzgerald JC et al (2016) Protocolized treatment is associated with decreased organ dysfunction in pediatric severe sepsis. Pediatr Crit Care Med 17:817-822 
35. Akcan Arikan A, Williams EA, Graf JM et al (2015) Resuscitation bundle in pediatric shock decreases acute kidney injury and improves outcomes. J Pediatr 167(1301-1305):e1

36. Cruz AT, Perry AM, Williams EA et al (2011) Implementation of goaldirected therapy for children with suspected sepsis in the emergency department. Pediatrics 127:e758-e766

37. Scott HF, Deakyne SJ, Woods JM et al (2015) The prevalence and diagnostic utility of systemic inflammatory response syndrome vital signs in a pediatric emergency department. Acad Emerg Med 22:381-389

38. Parshuram CS, Dryden-Palmer K, Farrell C, Canadian Critical Care Trials Group and the EPOCH Investigators et al (2018) Effect of a pediatric early warning system on all-cause mortality in hospitalized pediatric patients: The EPOCH randomized clinical trial. JAMA 319:1002-1012

39. Moore LJ, Jones SL, Kreiner LA et al (2009) Validation of a screening tool for the early identification of sepsis. J Trauma 66:1539-1546

40. Sepanski RJ, Godambe SA, Mangum CD et al (2014) Designing a pediatric severe sepsis screening tool. Front Pediatr 2:56

41. Balamuth F, Alpern ER, Abbadessa MK et al (2017) Improving recognition of pediatric severe sepsis in the emergency department: contributions of a vital sign-based electronic alert and bedside clinician identification. Ann Emerg Med 70(759-768):e2

42. Bradshaw C, Goodman I, Rosenberg R et al (2016) Implementation of an inpatient pediatric sepsis identification pathway. Pediatrics 137:e20144082

43. Balamuth F, Alpern ER, Grundmeier RW et al (2015) Comparison of two sepsis recognition methods in a pediatric emergency department. Acad Emerg Med 22:1298-1306

44. Schlapbach LJ, Weiss SL, Wolf J (2019) Reducing collateral damage from mandates for time to antibiotics in pediatric sepsis-primum non nocere. JAMA Pediatr 173:409-410

45. Leigh S, Grant A, Murray N et al (2019) The cost of diagnostic uncertainty: a prospective economic analysis of febrile children attending an NHS emergency department. BMC Med 17:48

46. Duncan H, Hutchison J, Parshuram CS (2006) The pediatric early warning system score: a severity of illness score to predict urgent medical need in hospitalized children. J Crit Care 21:271-278

47. Chapman SM, Wray J, Oulton K et al (2017) 'The Score Matters': wide variations in predictive performance of 18 paediatric track and trigger systems. Arch Dis Child 102:487-495

48. Lambert V, Matthews A, MacDonell R et al (2017) Paediatric early warning systems for detecting and responding to clinical deterioration in children: a systematic review. BMJ Open 7:e014497

49. Hernandez G, Bellomo R, Bakker J (2019) The ten pitfalls of lactate clearance in sepsis. Intensive Care Med 45:82-85

50. Rhodes A, Evans LE, Alhazzani W et al (2017) Surviving sepsis campaign: international guidelines for management of sepsis and septic shock: 2016. Intensive Care Med 43:304-377

51. Levy MM, Evans LE, Rhodes A (2018) The surviving sepsis campaign bundle: 2018 update. Intensive Care Med 44:925-928

52. Bai Z, Zhu X, Li M et al (2014) Effectiveness of predicting in-hospital mortality in critically ill children by assessing blood lactate levels at admission. BMC Pediatr 14:83

53. Chen M, Lu X, Hu L et al (2017) Development and validation of a mortality risk model for pediatric sepsis. Medicine (Baltimore) 96:e6923

54. Scott HF, Brou L, Deakyne SJ et al (2017) Association between early lactate levels and 30-day mortality in clinically suspected sepsis in children. JAMA Pediatr 171:249-255

55. Schlapbach $\sqcup$, MacLaren G, Straney L (2017) Venous vs arterial lactate and 30-day mortality in pediatric sepsis. JAMA Pediatr 171:813

56. Scott HF, Brou L, Deakyne SJ et al (2016) Lactate clearance and normalization and prolonged organ dysfunction in pediatric sepsis. J Pediatr 170(149-155):e1

57. Kortz TB, Axelrod DM, Chisti MJ et al (2017) Clinical outcomes and mortality before and after implementation of a pediatric sepsis protocol in a limited resource setting: a retrospective cohort study in Bangladesh. PLoS One 12:e0181160

58. Long E, Babl FE, Angley E et al (2016) A prospective quality improvement study in the emergency department targeting paediatric sepsis. Arch Dis Child 101:945-950
59. Workman JK, Ames SG, Reeder RW et al (2016) Treatment of pediatric septic shock with the surviving sepsis campaign guidelines and PICU patient outcomes. Pediatr Crit Care Med 17:e451-e458

60. Larsen GY, Mecham N, Greenberg R (2011) An emergency department septic shock protocol and care guideline for children initiated at triage. Pediatrics 127:e1585-e1592

61. Tuuri RE, Gehrig MG, Busch CE et al (2016) "Beat the Shock Clock": an interprofessional team improves pediatric septic shock care. Clin Pediatr (Phila) 55:626-638

62. McMullan BJ, Bowen A, Blyth CC et al (2016) Epidemiology and mortality of Staphylococcus aureus bacteremia in Australian and New Zealand children. JAMA Pediatr 170:979-986

63. Weiss SL, Fitzgerald JC, Balamuth F et al (2014) Delayed antimicrobial therapy increases mortality and organ dysfunction duration in pediatric sepsis. Crit Care Med 42:2409-2417

64. Martinón-Torres F, Salas A, Rivero-Calle I, EUCLIDS Consortium et al (2018) Life-threatening infections in children in Europe (the EUCLIDS project): a prospective cohort study. Lancet Child Adolesc Health 2:404-414

65. Agyeman PKA, Schlapbach LJ, Giannoni E, Swiss Pediatric Sepsis Study et al (2017) Epidemiology of blood culture-proven bacterial sepsis in children in Switzerland: A population-based cohort study. Lancet Child Adolesc Health 1:124-133

66. Lucignano B, Ranno S, Liesenfeld O et al (2011) Multiplex PCR allows rapid and accurate diagnosis of bloodstream infections in newborns and children with suspected sepsis. J Clin Microbiol 49:2252-2258

67. Rhee C, Filbin MR, Massaro AF, Centers for Disease Control and Prevention (CDC) Prevention Epicenters Program et al (2018) Compliance with the national SEP-1 quality measure and association with sepsis outcomes: a multicenter retrospective cohort study. Crit Care Med 46:1585-1591

68. Kumar A, Roberts D, Wood KE et al (2006) Duration of hypotension before initiation of effective antimicrobial therapy is the critical determinant of survival in human septic shock. Crit Care Med 34:1589-1596

69. Ferrer R, Martin-Loeches I, Phillips G et al (2014) Empiric antibiotic treatment reduces mortality in severe sepsis and septic shock from the first hour: results from a guideline-based performance improvement program. Crit Care Med 42:1749-1755

70. Barie PS, Hydo LJ, Shou J et al (2005) Influence of antibiotic therapy on mortality of critical surgical illness caused or complicated by infection. Surg Infect (Larchmt) 6:41-54

71. Barochia AV, Cui X, Vitberg D et al (2010) Bundled care for septic shock: an analysis of clinical trials. Crit Care Med 38:668-678

72. Gaieski DF, Mikkelsen ME, Band RA et al (2010) Impact of time to antibiotics on survival in patients with severe sepsis or septic shock in whom early goal-directed therapy was initiated in the emergency department. Crit Care Med 38:1045-1053

73. Klompas M, Calandra T, Singer M (2018) Antibiotics for sepsis-finding the equilibrium. JAMA 320:1433-1434

74. Mi MY, Klompas M, Evans L (2019) Early administration of antibiotics for suspected sepsis. N Engl J Med 380:593-596

75. Kumar A, Ellis P, Arabi Y, Cooperative Antimicrobial Therapy of Septic Shock Database Research Group et al (2009) Initiation of inappropriate antimicrobial therapy results in a fivefold reduction of survival in human septic shock. Chest 136:1237-1248

76. Kimberlin D, Brady M, Jackson M et al (2018) American Academy of Pediatrics Committee on Infectious Diseases Red Book ${ }^{\circledR}$ 2018. https:// redbook.solutions.aap.org/Book.aspx?bookid=2205. Accessed 18 Aug 2019

77. Sharland M, Butler K, Cant A et al (2016) European Society for Paediatric Infectious Diseases Manual of Childhood Infections-The Blue Book. https://global.oup.com/academic/product/manual-of-childhood-infec tions-9780198729228?cc=gb\&lang=en\&. Accessed 18 Aug 2019

78. Lehrnbecher T, Robinson P, Fisher B et al (2017) Guideline for the management of fever and neutropenia in children with cancer and hematopoietic stem-cell transplantation recipients: 2017 update. J Clin Oncol 35:2082-2094

79. Iroh Tam PY, Musicha P, Kawaza Ket al (2019) Emerging resistance to empiric antimicrobial regimens for pediatric bloodstream infections in Malawi (1998-2017). Clin Infect Dis 69:61-68 
80. Malosh RE, Martin ET, Heikkinen T et al (2018) Efficacy and safety of oseltamivir in children: systematic review and individual patient data meta-analysis of randomized controlled trials. Clin Infect Dis 66:1492-1500

81. Kumar A (2011) Early versus late oseltamivir treatment in severely ill patients with 2009 pandemic influenza A (H1N1): speed is life. J Antimicrob Chemother 66:959-963

82. Goodman KE, Lessler J, Cosgrove SE, Antibacterial Resistance Leadership Group et al (2016) a clinical decision tree to predict whether a bacteremic patient is infected with an extended-spectrum $\beta$-lactamaseproducing organism. Clin Infect Dis 63:896-903

83. Carapetis JR, Jacoby P, Carville K et al (2014) Effectiveness of clindamycin and intravenous immunoglobulin, and risk of disease in contacts, in invasive group a streptococcal infections. Clin Infect Dis 59:358-365

84. Guo Y, Gao W, Yang H et al (2016) De-escalation of empiric antibiotics in patients with severe sepsis or septic shock: a meta-analysis. Heart Lung 45:454-459

85. Hamdy RF, Zaoutis TE, Seo SK (2017) Antifungal stewardship considerations for adults and pediatrics. Virulence 8:658-672

86. Dierig A, Berger C, Agyeman PKA, Swiss Pediatric Sepsis Study et al (2018) Time-to-positivity of blood cultures in children with sepsis. Front Pediatr 6:222

87. Lin GL, McGinley JP, Drysdale SB et al (2018) Epidemiology and immune pathogenesis of viral sepsis. Front Immunol 9:2147

88. Paul M, Dickstein Y, Schlesinger A et al (2013) Beta-lactam versus betalactam-aminoglycoside combination therapy in cancer patients with neutropenia. Cochrane Database Syst Rev 6:CD003038

89. Paul M, Lador A, Grozinsky-Glasberg S et al (2014) Beta lactam antibiotic monotherapy versus beta lactam-aminoglycoside antibiotic combination therapy for sepsis. Cochrane Database Syst Rev 1:CD003344

90. Sjövall F, Perner A, Hylander Møller M (2017) Empirical mono- versus combination antibiotic therapy in adult intensive care patients with severe sepsis-a systematic review with meta-analysis and trial sequential analysis. J Infect 74:331-344

91. Baqui AH, Saha SK, Ahmed AS, Projahnmo Study Group in Bangladesh et al (2015) Safety and efficacy of alternative antibiotic regimens compared with 7 day injectable procaine benzylpenicillin and gentamicin for outpatient treatment of neonates and young infants with clinical signs of severe infection when referral is not possible: a randomised, open-label, equivalence trial. Lancet Glob Health 3:e279-e287

92. Metsvaht T, IImoja ML, Parm Ü et al (2010) Comparison of ampicillin plus gentamicin vs. penicillin plus gentamicin in empiric treatment of neonates at risk of early onset sepsis. Acta Paediatr 99:665-672

93. Pasha YZ, Ahmadpour-Kacho M, Behmadi R et al (2014) 3-day versus 5-day course of intravenous antibiotics for suspected early onset neonatal sepsis: a randomized controlled trial. Iran J Pediatr 24:673-678

94. Ramasamy S, Biswal N, Bethou A et al (2014) Comparison of two empiric antibiotic regimen in late onset neonatal sepsis-a randomized controlled trial. J Trop Pediatr 60:83-86

95. Taheri PA, Eslamieh H, Salamati $P$ (2011) Is ceftizoxime an appropriate surrogate for amikacin in neonatal sepsis treatment? A randomized clinical trial. Acta Med Iran 49:499-503

96. Tewari W, Jain N (2014) Monotherapy with amikacin or piperacillintazobactum empirically in neonates at risk for early-onset sepsis: a randomized controlled trial. J Trop Pediatr 60:297-302

97. Tshefu A, Lokangaka A, Ngaima S, African Neonatal Sepsis Trial (AFRINEST) group et al (2015) Simplified antibiotic regimens compared with injectable procaine benzylpenicillin plus gentamicin for treatment of neonates and young infants with clinical signs of possible serious bacterial infection when referral is not possible: a randomised, openlabel, equivalence trial. Lancet 385:1767-1776

98. Zaidi AK, Tikmani SS, Warraich HJ et al (2012) Community-based treatment of serious bacterial infections in newborns and young infants: a randomized controlled trial assessing three antibiotic regimens. Pediatr Infect Dis J 31:667-672

99. Ibrahim SL, Zhang L, Brady TM et al (2015) Low-dose gentamicin for uncomplicated enterococcus faecalis bacteremia may be nephrotoxic in children. Clin Infect Dis 61:1119-1124

100. Tamma PD, Turnbull AE, Harris AD et al (2013) Less is more: combination antibiotic therapy for the treatment of gram-negative bacteremia in pediatric patients. JAMA Pediatr 167:903-910
101. Falagas ME, Lourida P, Poulikakos P et al (2014) Antibiotic treatment of infections due to carbapenem-resistant Enterobacteriaceae: systematic evaluation of the available evidence. Antimicrob Agents Chemother 58:654-663

102. Bass SN, Bauer SR, Neuner EA et al (2015) Impact of combination antimicrobial therapy on mortality risk for critically ill patients with carbapenem-resistant bacteremia. Antimicrob Agents Chemother 59:3748-3753

103. Chiotos K, Tamma PD, Flett KB et al (2018) Increased 30-day mortality associated with carbapenem-resistant Enterobacteriaceae in children. Open Forum Infect Dis 5:ofy222

104. Baltimore RS, Gewitz M, Baddour LM, American Heart Association Rheumatic Fever, Endocarditis, and Kawasaki Disease Committee of the Council on Cardiovascular Disease in the Young and the Council on Cardiovascular and Stroke Nursing et al (2015) Infective endocarditis in childhood: 2015 update: A scientific statement from the American Heart Association. Circulation 132:1487-1515

105. Hanretty AM, Kaur I, Evangelista AT et al (2018) Pharmacokinetics of the meropenem component of meropenem-vaborbactam in the treatment of KPC-producing Klebsiella pneumoniae bloodstream infection in a pediatric patient. Pharmacotherapy 38:e87-e91

106. Costa Pde O, Atta EH, Silva AR (2015) Infection with multidrug-resistant gram-negative bacteria in a pediatric oncology intensive care unit: risk factors and outcomes. J Pediatr (Rio J) 91:435-441

107. Shankar K, Radhakrishnan V, Vijayakumar V et al (2018) Prevalence of multi-drug resistant organisms in stool of paediatric patients with acute leukaemia and correlation with blood culture positivity: a single institution experience. Pediatr Blood Cancer 65

108. Pereira CA, Petrilli AS, Carlesse FA et al (2009) Cefepime monotherapy is as effective as ceftriaxone plus amikacin in pediatric patients with cancer and high-risk febrile neutropenia in a randomized comparison. J Microbiol Immunol Infect 42:141-147

109. Petrilli AS, Cypriano M, Dantas LS et al (2003) Evaluation of ticarcillin/clavulanic acid versus ceftriaxone plus amikacin for fever and neutropenia in pediatric patients with leukemia and lymphoma. Braz J Infect Dis 7:111-120

110. Daneman N, Low DE, McGeer A et al (2008) At the threshold: defining clinically meaningful resistance thresholds for antibiotic choice in community-acquired pneumonia. Clin Infect Dis 46:1131-1138

111. Mandell LA, Wunderink RG, Anzueto A, Infectious Diseases Society of America; American Thoracic Society et al (2007) Infectious Diseases Society of America/American Thoracic Society consensus quidelines on the management of community-acquired pneumonia in adults. Clin Infect Dis 44(Suppl 2):S27-S72

112. Liu C, Bayer A, Cosgrove SE, Infectious Diseases Society of America et al (2011) Clinical practice guidelines by the Infectious Diseases Society of America for the treatment of methicillin-resistant Staphylococcus aureus infections in adults and children. Clin Infect Dis 52:e18-e55

113. Cies JJ, Moore WS 2nd, Enache A et al (2018) $\beta$-lactam therapeutic drug management in the PICU. Crit Care Med 46:272-279

114. Nehus EJ, Mizuno T, Cox S et al (2016) Pharmacokinetics of meropenem in children receiving continuous renal replacement therapy: validation of clinical trial simulations. J Clin Pharmacol 56:291-297

115. Guilhaumou R, Benaboud S, Bennis Y et al (2019) Optimization of the treatment with beta-lactam antibiotics in critically ill patientsguidelines from the French Society of Pharmacology and Therapeutics (Société Française de Pharmacologie et Thérapeutique-SFPT) and the French Society of Anaesthesia and Intensive Care Medicine (Société Française d'Anesthésie et Réanimation-SFAR). Crit Care 23:104

116. Roberts JA, Abdul-Aziz MH, Lipman J, International Society of AntiInfective Pharmacology and the Pharmacokinetics and Pharmacodynamics Study Group of the European Society of Clinical Microbiology and Infectious Diseases et al (2014) Individualised antibiotic dosing for patients who are critically ill: challenges and potential solutions. Lancet Infect Dis 14:498-509

117. Udy AA, Varghese JM, Altukroni M et al (2012) Subtherapeutic initial $\beta$-lactam concentrations in select critically ill patients: association between augmented renal clearance and low trough drug concentrations. Chest 142:30-39

118. Cotta MO, Roberts JA, Lipman J (2015) Antibiotic dose optimization in critically ill patients. Med Intensiva 39:563-572 
119. De Bleecker JL, Meire VI, Declercq W et al (1999) Immunolocalization of tumor necrosis factor-alpha and its receptors in inflammatory myopathies. Neuromuscul Disord 9:239-246

120. Van Der Heggen T, Dhont E, Peperstraete H et al (2019) Augmented renal clearance: a common condition in critically ill children. Pediatr Nephrol 34:1099-1106

121. Rizk NA, Kanafani ZA, Tabaja HZ et al (2017) Extended infusion of betalactam antibiotics: optimizing therapy in critically-ill patients in the era of antimicrobial resistance. Expert Rev Anti Infect Ther 15:645-652

122. Demirjian A, Finkelstein Y, Nava-Ocampo A et al (2013) A randomized controlled trial of a vancomycin loading dose in children. Pediatr Infect Dis J 32:1217-1223

123. Godbout EJ, Pakyz AL, Markley JD et al (2018) Pediatric antimicrobial stewardship: state of the art. Curr Infect Dis Rep 20:39

124. Weiss CH, Persell SD, Wunderink RG et al (2012) Empiric antibiotic, mechanical ventilation, and central venous catheter duration as potential factors mediating the effect of a checklist prompting intervention on mortality: an exploratory analysis. BMC Health Serv Res 12:198

125. Weiss CH, Moazed F, McEvoy CA et al (2011) Prompting physicians to address a daily checklist and process of care and clinical outcomes: a single-site study. Am J Respir Crit Care Med 184:680-686

126. Schuetz P, Briel M, Christ-Crain M et al (2012) Procalcitonin to guide initiation and duration of antibiotic treatment in acute respiratory infections: an individual patient data meta-analysis. Clin Infect Dis 55:651-662

127. Matthaiou DK, Ntani G, Kontogiorgi M et al (2012) An ESICM systematic review and meta-analysis of procalcitonin-guided antibiotic therapy algorithms in adult critically ill patients. Intensive Care Med 38:940-949

128. Prkno A, Wacker C, Brunkhorst FM et al (2013) Procalcitonin-guided therapy in intensive care unit patients with severe sepsis and septic shock - a systematic review and meta-analysis. Crit Care 17:R291

129. Westwood M, Ramaekers B, Whiting P et al (2015) Procalcitonin testing to guide antibiotic therapy for the treatment of sepsis in intensive care settings and for suspected bacterial infection in emergency department settings: a systematic review and cost-effectiveness analysis. Health Technol Assess 19:v-xxv, 1-236

130. Wacker C, Prkno A, Brunkhorst FM et al (2013) Procalcitonin as a diagnostic marker for sepsis: a systematic review and meta-analysis. Lancet Infect Dis 13:426-435

131. de Jong E, van Oers JA, Beishuizen A et al (2016) Efficacy and safety of procalcitonin guidance in reducing the duration of antibiotic treatment in critically ill patients: a randomised, controlled, open-label trial. Lancet Infect Dis 16:819-827

132. Stocker M, van Herk W, El Helou S, NeoPInS Study Group et al (2017) Procalcitonin-guided decision making for duration of antibiotic therapy in neonates with suspected early-onset sepsis: a multicentre, randomised controlled trial (NeoPIns). Lancet 390:871-881

133. Public Health England (2015) Start Smart-Then Focus. United Kingdom, Public Health England. https://www.gov.uk/government/publi cations/antimicrobial-stewardship-start-smart-then-focus\#history. Accessed 18 Aug 2019

134. Bamberger DM (1996) Outcome of medical treatment of bacterial abscesses without therapeutic drainage: review of cases reported in the literature. Clin Infect Dis 23:592-603

135. Osmon DR, Berbari EF, Berendt AR, Infectious Diseases Society of America et al (2013) Diagnosis and management of prosthetic joint infection: clinical practice guidelines by the Infectious Diseases Society of America. Clin Infect Dis 56:e1-e25

136. Baddour LM, Wilson WR, Bayer AS, American Heart Association Committee on Rheumatic Fever, Endocarditis, and Kawasaki Disease of the Council on Cardiovascular Disease in the Young, Council on Clinical Cardiology, Council on Cardiovascular Surgery and Anesthesia, and Stroke Council et al (2015) Infective endocarditis in adults: Diagnosis, antimicrobial therapy, and management of complications: a scientific statement for healthcare professionals from the American Heart Association. Circulation 132:1435-1486

137. Yahav D, Franceschini E, Koppel F, Bacteremia Duration Study Group et al (2019) Seven versus 14 days of antibiotic therapy for uncomplicated gram-negative bacteremia: a noninferiority randomized controlled trial. Clin Infect Dis 69:1091-1098
138. Chotiprasitsakul D, Han JH, Cosgrove SE, Antibacterial Resistance Leadership Group et al (2018) Comparing the outcomes of adults with Enterobacteriaceae bacteremia receiving short-course versus prolonged-course antibiotic therapy in a multicenter, propensity scorematched cohort. Clin Infect Dis 66:172-177

139. Chong YP, Moon SM, Bang KM et al (2013) Treatment duration for uncomplicated Staphylococcus aureus bacteremia to prevent relapse: analysis of a prospective observational cohort study. Antimicrob Agents Chemother 57:1150-1156

140. Chowdhary G, Dutta S, Narang A (2006) Randomized controlled trial of 7-day vs. 14-day antibiotics for neonatal sepsis. J Trop Pediatr 52:427-432

141. Liu C, Bayer A, Cosgrove SE et al (2011) Clinical practice guidelines by the Infectious Diseases Society of America for the treatment of methicillin-resistant Staphylococcus aureus infections in adults and children: executive summary. Clin Infect Dis 52:285-292

142. Berrevoets MAH, Kouijzer IJE, Aarntzen EHJG et al (2017) 18F-FDG PET/ CT optimizes treatment in Staphylococcus Aureus bacteremia and is associated with reduced mortality. J Nucl Med 58:1504-1510

143. McMullan BJ, Andresen D, Blyth CC, ANZPID-ASAP group et al (2016) Antibiotic duration and timing of the switch from intravenous to oral route for bacterial infections in children: systematic review and guidelines. Lancet Infect Dis 16:e139-e152

144. Alexander VN, Northrup V, Bizzarro MJ (2011) Antibiotic exposure in the newborn intensive care unit and the risk of necrotizing enterocolitis. $J$ Pediatr 159:392-397

145. Paganini H, Rodriguez Brieshcke T, Santos P et al (2002) Risk factors for nosocomial candidaemia: a case-control study in children. J Hosp Infect 50:304-308

146. Zaoutis TE, Prasad PA, Localio AR et al (2010) Risk factors and predictors for candidemia in pediatric intensive care unit patients: implications for prevention. Clin Infect Dis 51:e38-e45

147. Chatterjee A, Modarai M, Naylor NR et al (2018) Quantifying drivers of antibiotic resistance in humans: a systematic review. Lancet Infect Dis 18:e368-e378

148. Brown KA, Khanafer N, Daneman N et al (2013) Meta-analysis of antibiotics and the risk of community-associated Clostridium difficile infection. Antimicrob Agents Chemother 57:2326-2332

149. Chuang YY, Huang YC, Lee CY et al (2004) Methicillin-resistant Staphylococcus aureus bacteraemia in neonatal intensive care units: an analysis of 90 episodes. Acta Paediatr 93:786-790

150. Couto RC, Barbosa JA, Pedrosa TM et al (2007) C-reactive proteinguided approach may shorten length of antimicrobial treatment of culture-proven late-onset sepsis: an intervention study. Braz J Infect Dis 11:240-245

151. Dimopoulos G, Matthaiou DK, Karageorgopoulos DE et al (2008) Shortversus long-course antibacterial therapy for community-acquired pneumonia: a meta-analysis. Drugs 68:1841-1854

152. Havey TC, Fowler RA, Daneman N (2011) Duration of antibiotic therapy for bacteremia: a systematic review and meta-analysis. Crit Care 15:R267

153. Hemels MA, van den Hoogen A, Verboon-Maciolek MA et al (2012) Shortening the antibiotic course for the treatment of neonatal coagulase-negative staphylococcal sepsis: fine with three days? Neonatology 101:101-105

154. Karageorgopoulos DE, Valkimadi PE, Kapaskelis A et al (2009) Short versus long duration of antibiotic therapy for bacterial meningitis: a meta-analysis of randomised controlled trials in children. Arch Dis Child 94:607-614

155. Kavaliotis J, Manios SG, Kansouzidou A et al (1989) Treatment of childhood bacterial meningitis with ceftriaxone once daily: open, prospective, randomized, comparative study of short-course versus standardlength therapy. Chemotherapy 35:296-303

156. Lassi ZS, Imdad A, Bhutta ZA (2017) Short-course versus long-course intravenous therapy with the same antibiotic for severe communityacquired pneumonia in children aged two months to 59 months. Cochrane Database Syst Rev 10:CD008032

157. Lin TY, Chrane DF, Nelson JD et al (1985) Seven days of ceftriaxone therapy is as effective as ten days' treatment for bacterial meningitis. JAMA 253:3559-3563 
158. Linder N, Lubin D, Hernandez A et al (2013) Duration of vancomycin treatment for coagulase-negative Staphylococcus sepsis in very low birth weight infants. Br J Clin Pharmacol 76:58-64

159. Martin E, Hohl P, Guggi T et al (1990) Short course single daily ceftriaxone monotherapy for acute bacterial meningitis in children: results of a Swiss multicenter study. Part I: Clinical results. Infection 18:70-77

160. Molyneux E, Nizami SQ, Saha S, CSF 5 Study Group et al (2011) 5 versus 10 days of treatment with ceftriaxone for bacterial meningitis in children: a double-blind randomised equivalence study. Lancet 377:1837-1845

161. Onakpoya IJ, Walker AS, Tan PS et al (2018) Overview of systematic reviews assessing the evidence for shorter versus longer duration antibiotic treatment for bacterial infections in secondary care. PLoS One 13:e0194858

162. Park SH, Milstone AM, Diener-West M et al (2014) Short versus prolonged courses of antibiotic therapy for children with uncomplicated Gram-negative bacteraemia. J Antimicrob Chemother 69:779-785

163. Rohatgi S, Dewan P, Faridi MMA et al (2017) Seven versus 10 days antibiotic therapy for culture-proven neonatal sepsis: a randomised controlled trial. J Paediatr Child Health 53:556-562

164. Roine I, Ledermann W, Foncea LM et al (2000) Randomized trial of four vs. seven days of ceftriaxone treatment for bacterial meningitis in children with rapid initial recovery. Pediatr Infect Dis J 19:219-222

165. Singhi P, Kaushal M, Singhi S et al (2002) Seven days vs. 10 days ceftriaxone therapy in bacterial meningitis. J Trop Pediatr 48:273-279

166. Tsai MH, Huang YC, Chiu CH et al (2007) Nontyphoidal Salmonella bacteremia in previously healthy children: analysis of 199 episodes. Pediatr Infect Dis J 26:909-913

167. Yen MH, Huang YC, Chiu CH et al (2002) Duration of antimicrobial therapy for non-typhoid Salmonella bacteremia in healthy children. J Microbiol Immunol Infect 35:94-98

168. Jernelius H, Zbornik J, Bauer CA (1988) One or three weeks'treatment of acute pyelonephritis? A double-blind comparison, using a fixed combination of pivampicillin plus pivmecillinam. Acta Med Scand 223:469-477

169. Pakistan Multicentre Amoxycillin Short Course Therapy (MASCOT) pneumonia study group (2002) Clinical efficacy of 3 days versus 5 days of oral amoxicillin for treatment of childhood pneumonia: a multicentre double-blind trial. Lancet 360:835-841

170. Agarwal G, Awasthi S, Kabra SK, ISCAP Study Group et al (2004) Three day versus five day treatment with amoxicillin for non-severe pneumonia in young children: a multicentre randomised controlled trial. BMJ 328:791

171. Lagunes L, Encina B, Ramirez-Estrada S (2016) Current understanding in source control management in septic shock patients: a review. Ann Transl Med 4:330

172. Martínez ML, Ferrer R, Torrents E, Edusepsis Study Group et al (2017) Impact of source control in patients with severe sepsis and septic shock. Crit Care Med 45:11-19

173. Fustes-Morales A, Gutierrez-Castrellon P, Duran-Mckinster C et al (2002) Necrotizing fasciitis: report of 39 pediatric cases. Arch Dermatol 138:893-899

174. Bingöl-Koloğlu M, Yildiz RV, Alper B et al (2007) Necrotizing fasciitis in children: diagnostic and therapeutic aspects. J Pediatr Surg 42:1892-1897

175. Endorf FW, Garrison MM, Klein MB et al (2012) Characteristics, therapies, and outcome of children with necrotizing soft tissue infections. Pediatr Infect Dis J 31:221-223

176. Nazemi KJ, Buescher ES, Kelly RE Jr et al (2003) Central venous catheter removal versus in situ treatment in neonates with enterobacteriaceae bacteremia. Pediatrics 111:e269-e274

177. Vasudevan C, Oddie SJ, McGuire W (2016) Early removal versus expectant management of central venous catheters in neonates with bloodstream infection. Cochrane Database Syst Rev 4:CD008436

178. Rodriguez D, Park BJ, Almirante B, Barcelona Candidemia Project Study Group et al (2007) Impact of early central venous catheter removal on outcome in patients with candidaemia. Clin Microbiol Infect 13:788-793

179. Smith PB, Benjamin DK Jr, Cotten CM et al (2008) Is an increased dwell time of a peripherally inserted catheter associated with an increased risk of bloodstream infection in infants? Infect Control Hosp Epidemiol 29:749-753

180. Santhanam I, Sangareddi S, Venkataraman S et al (2008) A prospective randomized controlled study of two fluid regimens in the initial management of septic shock in the emergency department. Pediatr Emerg Care 24:647-655

181. Sankar J, Ismail J, Sankar MJ et al (2017) Fluid bolus over 15-20 versus 5-10 minutes each in the first hour of resuscitation in children with septic shock: a randomized controlled trial. Pediatr Crit Care Med 18:e435-e445

182. Inwald DP, Canter R, Woolfall K, PERUKI (Paediatric Emergency Research in the UK and Ireland) and PICS SG (Paediatric Intensive Care Society Study Group) et al (2019) Restricted fluid bolus volume in early septic shock: Results of the fluids in shock pilot trial. Arch Dis Child 104:426-431

183. Maitland K, Kiguli S, Opoka RO, FEAST Trial Group et al (2011) Mortality after fluid bolus in African children with severe infection. N Engl J Med 364:2483-2495

184. Carcillo JA, Davis AL, Zaritsky A (1991) Role of early fluid resuscitation in pediatric septic shock. JAMA 266:1242-1245

185. Han YY, Carcillo JA, Dragotta MA et al (2003) Early reversal of pediatricneonatal septic shock by community physicians is associated with improved outcome. Pediatrics 112:793-799

186. Carcillo JA, Kuch BA, Han YY et al (2009) Mortality and functional morbidity after use of PALS/APLS by community physicians. Pediatrics 124:500-508

187. van Paridon BM, Sheppard C, Garcia GG et al (2015) Timing of antibiotics, volume, and vasoactive infusions in children with sepsis admitted to intensive care. Crit Care 19:293

188. Houston KA, George EC, Maitland K (2018) Implications for paediatric shock management in resource-limited settings: a perspective from the FEAST trial. Crit Care 22:119

189. Voigt J, Waltzman M, Lottenberg L (2012) Intraosseous vascular access for in-hospital emergency use: a systematic clinical review of the literature and analysis. Pediatr Emerg Care 28:185-199

190. de Oliveira CF, de Oliveira DS, Gottschald AF et al (2008) ACCM/PALS haemodynamic support guidelines for paediatric septic shock: an outcomes comparison with and without monitoring central venous oxygen saturation. Intensive Care Med 34:1065-1075

191. Arya B, Kerstein D, Leu CS et al (2016) Echocardiographic assessment of right atrial pressure in a pediatric and young adult population. Pediatr Cardiol 37:558-567

192. Semler MW, Self WH, Wanderer JP, SMART Investigators and the Pragmatic Critical Care Research Group et al (2018) Balanced crystalloids versus saline in critically ill adults. N Engl J Med 378:829-839

193. Weiss SL, Keele L, Balamuth F et al (2017) Crystalloid fluid choice and clinical outcomes in pediatric sepsis: a matched retrospective cohort study. J Pediatr 182(304-310):e10

194. Emrath ET, Fortenberry JD, Travers C et al (2017) Resuscitation with balanced fluids is associated with improved survival in pediatric severe sepsis. Crit Care Med 45:1177-1183

195. SelfWH, Semler MW, Wanderer JP, SALT-ED Investigators et al (2018) balanced crystalloids versus saline in noncritically ill adults. N Engl J Med 378:819-828

196. Perner A, Haase N, Guttormsen AB et al, 6S Trial Group, Scandinavian Critical Care Trials Group (2012) Hydroxyethyl starch 130/042 versus ringer's acetate in severe sepsis. N Engl I Med 367:124-134

197. Myburgh JA, Finfer S, Bellomo R, CHEST Investigators; Australian and New Zealand Intensive Care Society Clinical Trials Group et al (2012) Hydroxyethyl starch or saline for fluid resuscitation in intensive care. N Engl J Med 367:1901-1911

198. Zarychanski R, Abou-Setta AM, Turgeon AF et al (2013) Association of hydroxyethyl starch administration with mortality and acute kidney injury in critically ill patients requiring volume resuscitation: a systematic review and meta-analysis. JAMA 309:678-688

199. McIntyre L (2012) Hydroxyethyl starch for fluid resuscitation. https://crit calcarecanada.com/presentations/2012/hydroxyethyl_starch_for_fluid _resuscitation.pdf. Accessed 18 Aug 2019

200. European Medicines Agency (2018) Hydroxyethyl-starch solutions for infusion to be suspended-CMDh endorses PRAC recommendation. https://www.ema.europa.eu/en/news/hydroxyethyl-starch-solut 
ions-infusion-be-suspended-cmdh-endorses-prac-recommendation. Accessed 18 Aug 2019

201. Upadhyay M, Singhi S, Murlidharan J et al (2005) Randomized evaluation of fluid resuscitation with crystalloid (saline) and colloid (polymer from degraded gelatin in saline) in pediatric septic shock. Indian Pediatr 42:223-231

202. Lamontagne F, Day AG, Meade MO et al (2018) Pooled analysis of higher versus lower blood pressure targets for vasopressor therapy septic and vasodilatory shock. Intensive Care Med 44:12-21

203. Davis AL, Carcillo JA, Aneja RK et al (2017) American College of Critical Care Medicine clinical practice parameters for hemodynamic support of pediatric and neonatal septic shock. Crit Care Med 45:1061-1093

204. Tibby SM, Hatherill M, Marsh MJ et al (1997) Clinicians' abilities to estimate cardiac index in ventilated children and infants. Arch Dis Child 77:516-518

205. Brierley J, Peters MJ (2008) Distinct hemodynamic patterns of septic shock at presentation to pediatric intensive care. Pediatrics 122:752-759

206. Egan JR, Festa M, Cole AD et al (2005) Clinical assessment of cardiac performance in infants and children following cardiac surgery. Intensive Care Med 31:568-573

207. Ranjit S, Kissoon N (2013) Bedside echocardiography is useful in assessing children with fluid and inotrope resistant septic shock. Indian J Crit Care Med 17:224-230

208. Ranjit S, Aram G, Kissoon N et al (2014) Multimodal monitoring for hemodynamic categorization and management of pediatric septic shock: a pilot observational study. Pediatr Crit Care Med 15:e17-e26

209. Razavi A, Newth CJL, Khemani RG et al (2017) Cardiac output and systemic vascular resistance: clinical assessment compared with a noninvasive objective measurement in children with shock. J Crit Care 39:6-10

210. El-Nawawy AA, Abdelmohsen AM, Hassouna HM (2018) Role of echocardiography in reducing shock reversal time in pediatric septic shock: a randomized controlled trial. J Pediatr (Rio J) 94:31-39

211. Gan H, Cannesson M, Chandler JR et al (2013) Predicting fluid responsiveness in children: a systematic review. Anesth Analg 117:1380-1392

212. Rivers E, Nguyen B, Havstad S, Early Goal-Directed Therapy Collaborative Group et al (2001) Early goal-directed therapy in the treatment of severe sepsis and septic shock. N Engl J Med 345:1368-1377

213. Peake SL, Delaney A, Bailey M, ARISE Investigators, ANZICS Clinical Trials Group et al (2014) Goal-directed resuscitation for patients with early septic shock. N Engl J Med 371:1496-1506

214. Yealy DM, Kellum JA, Huang DT, ProCESS Investigators et al (2014) A randomized trial of protocol-based care for early septic shock. N Engl J Med 370:1683-1693

215. Mouncey PR, Osborn TM, Power GS, ProMISe Trial Investigators et al (2015) Trial of early, goal-directed resuscitation for septic shock. N Engl J Med 372:1301-1311

216. Jansen TC, van Bommel J, Schoonderbeek FJ, LACTATE study group et al (2010) Early lactate-guided therapy in intensive care unit patients: a multicenter, open-label, randomized controlled trial. Am J Respir Crit Care Med 182:752-761

217. Jones AE, Shapiro NI, Trzeciak S, Emergency Medicine Shock Research Network (EMShockNet) Investigators et al (2010) Lactate clearance vs central venous oxygen saturation as goals of early sepsis therapy: a randomized clinical trial. JAMA 303:739-746

218. Tian HH, Han SS, LV CJ, et al (2012) The effect of early goal lactate clearance rate on the outcome of septic shock patients with severe pneumonia. Zhongguo Wei Zhong Bing Ji Jiu Yi Xue 24:42-45

219. Yu B, Tian HY, Hu ZJ, et al (2013) Comparison of the effect of fluid resuscitation as guided either by lactate clearance rate or by central venous oxygen saturation in patients with sepsis. Zhonghua Wei Zhong Bing Ji Jiu Yi Xue 25:578-583

220. Lyu X, Xu Q, Cai G et al (2015) Efficacies of fluid resuscitation as guided by lactate clearance rate and central venous oxygen saturation in patients with septic shock. Zhonghua Yi Xue Za Zhi 95:496-500

221. Zhou X, Liu D, Su L et al (2017) Use of stepwise lactate kinetics-oriented hemodynamic therapy could improve the clinical outcomes of patients with sepsis-associated hyperlactatemia. Crit Care 21:33
222. Ventura AM, Shieh HH, Bousso A et al (2015) Double-blind prospective randomized controlled trial of dopamine versus epinephrine as first-line vasoactive drugs in pediatric septic shock. Crit Care Med 43:2292-2302

223. Ramaswamy KN, Singhi S, Jayashree M et al (2016) Double-blind randomized clinical trial comparing dopamine and epinephrine in pediatric fluid-refractory hypotensive septic shock. Pediatr Crit Care Med 17:e502-e512

224. Piva J, Alquati T, Garcia PC et al (1992) Norepinephrine infusion increases urine output in children under sedative and analgesic infusion. Rev Assoc Med Bras 2014(60):208-215

225. Avni T, Lador A, Lev S et al (2015) Vasopressors for the treatment of septic shock: systematic review and meta-analysis. PLoS One 10:e0129305

226. Arikan AA, Zappitelli M, Goldstein SL et al (2012) Fluid overload is associated with impaired oxygenation and morbidity in critically ill children. Pediatr Crit Care Med 13:253-258

227. Lampin ME, Rousseaux J, Botte A et al (2012) Noradrenaline use for septic shock in children: doses, routes of administration and complications. Acta Paediatr 101:e426-e430

228. Patregnani JT, Sochet AA, Klugman D (2017) Short-term peripheral vasoactive infusions in pediatrics: where is the harm? Pediatr Crit Care Med 18:e378-e381

229. Choong K, Bohn D, Fraser DD, Canadian Critical Care Trials Group et al (2009) Vasopressin in pediatric vasodilatory shock: a multicenter rand omized controlled trial. Am J Respir Crit Care Med 180:632-639

230. Baldasso E, Ramos Garcia PC, Piva JP et al (2007) Hemodynamic and metabolic effects of vasopressin infusion in children with shock. J Pediatr (Rio J) 83:S137-S145

231. Yildizdas D, Yapicioglu H, Celik U et al (2008) Terlipressin as a rescue therapy for catecholamine-resistant septic shock in children. Intensive Care Med 34:511-517

232. Mclntyre WF, Um KJ, Alhazzani W et al (2018) Association of vasopressin plus catecholamine vasopressors vs catecholamines alone with atrial fibrillation in patients with distributive shock: a systematic review and meta-analysis. JAMA 319:1889-1900

233. Papoff $P$, Caresta $E$, Versacci $P$ et al (2012) Beneficial effects of levosimendan in infants with sepsis-associated cardiac dysfunction: report of 2 cases. Pediatr Emerg Care 28:1062-1065

234. Rich N, West N, McMaster P et al (2003) Milrinone in meningococcal sepsis. Pediatr Crit Care Med 4:394-395

235. Pham T, Brochard LJ, Slutsky AS (2017) Mechanical ventilation: state of the art. Mayo Clin Proc 92:1382-1400

236. Aubier M, Viires N, Syllie G et al (1982) Respiratory muscle contribution to lactic acidosis in low cardiac output. Am Rev Respir Dis 126:648-652

237. Cheifetz IM (2003) Invasive and noninvasive pediatric mechanical ventilation. Respir Care 48:442-453

238. Ghuman AK, Newth CJ, Khemani RG (2012) The association between the end tidal alveolar dead space fraction and mortality in pediatric acute hypoxemic respiratory failure. Pediatr Crit Care Med 13:11-15

239. Khemani RG, Smith L, Lopez-Fernandez YM, Pediatric Acute Respiratory Distress syndrome Incidence and Epidemiology (PARDIE) Investigators; Pediatric Acute Lung Injury and Sepsis Investigators (PALISI) Network et al (2019) Paediatric acute respiratory distress syndrome incidence and epidemiology (PARDIE): an international, observational study. Lancet Respir Med 7:115-128

240. Volpicelli G, Elbarbary M, Blaivas M, International Liaison Committee on Lung Ultrasound (ILC-LUS) for International Consensus Conference on Lung Ultrasound (ICC-LUS) et al (2012) International evidence-based recommendations for point-of-care lung ultrasound. Intensive Care Med 38:577-591

241. Riviello ED, Kiviri W, Twagirumugabe T et al (2016) Hospital incidence and outcomes of the acute respiratory distress syndrome using the Kigali modification of the berlin definition. Am J Respir Crit Care Med 193:52-59

242. De Luca D, van Kaam AH, Tingay DG et al (2017) The montreux definition of neonatal ARDS: biological and clinical background behind the description of a new entity. Lancet Respir Med 5:657-666

243. See KC, Ong V, Tan YL et al (2018) Chest radiography versus lung ultrasound for identification of acute respiratory distress syndrome: a retrospective observational study. Crit Care 22:203

244. Watt I, Ledingham IM (1984) Mortality amongst multiple trauma patients admitted to an intensive therapy unit. Anaesthesia 39:973-981 
245. den Brinker M, Joosten KF, Liem O et al (2005) Adrenal insufficiency in meningococcal sepsis: bioavailable cortisol levels and impact of interleukin-6 levels and intubation with etomidate on adrenal function and mortality. J Clin Endocrinol Metab 90:5110-5117

246. Absalom A, Pledger D, Kong A (1999) Adrenocortical function in critically ill patients $24 \mathrm{~h}$ after a single dose of etomidate. Anaesthesia $54: 861-867$

247. Schenarts CL, Burton JH, Riker RR (2001) Adrenocortical dysfunction following etomidate induction in emergency department patients. Acad Emerg Med 8:1-7

248. Hildreth AN, Mejia VA, Maxwell RA et al (2008) Adrenal suppression following a single dose of etomidate for rapid sequence induction: a prospective randomized study. J Trauma 65:573-579

249. Jabre P, Combes X, Lapostolle F, KETASED Collaborative Study Group et al (2009) Etomidate versus ketamine for rapid sequence intubation in acutely ill patients: a multicentre randomised controlled trial. Lancet 374:293-300

250. Muñoz-Bonet Jl, Flor-Macián EM, Brines J et al (2010) Predictive factors for the outcome of noninvasive ventilation in pediatric acute respiratory failure. Pediatr Crit Care Med 11:675-680

251. James CS, Hallewell CP, James DP et al (2011) Predicting the success of non-invasive ventilation in preventing intubation and re-intubation in the paediatric intensive care unit. Intensive Care Med 37:1994-2001

252. Wolfler A, Calderini E, lannella E, Network of Pediatric Intensive Care Unit Study Group et al (2015) Evolution of noninvasive mechanical ventilation use: A cohort study among Italian PICUs. Pediatr Crit Care Med 16:418-427

253. Abadesso C, Nunes P, Silvestre C et al (2012) Non-invasive ventilation in acute respiratory failure in children. Pediatr Rep 4:e16

254. Dohna-Schwake C, Stehling F, Tschiedel E et al (2011) Non-invasive ventilation on a pediatric intensive care unit: feasibility, efficacy, and predictors of success. Pediatr Pulmonol 46:1114-1120

255. Piastra M, De Luca D, Marzano L et al (2011) The number of failing organs predicts non-invasive ventilation failure in children with ALI/ ARDS. Intensive Care Med 37:1510-1516

256. Yaman A, Kendirli T, Ödek Ç et al (2016) Efficacy of noninvasive mechanical ventilation in prevention of intubation and reintubation in the pediatric intensive care unit. J Crit Care 32:175-181

257. Pancera CF, Hayashi M, Fregnani JH et al (2008) Noninvasive ventilation in immunocompromised pediatric patients: eight years of experience in a pediatric oncology intensive care unit. J Pediatr Hematol Oncol 30:533-538

258. Peters MJ, Agbeko R, Davis P, SCARF Study Investigators and the Pediatric Intensive Care Society Study Group (PICS-SG) et al (2018) Randomized study of early continuous positive airways pressure in acute respiratory failure in children with impaired immunity (SCARF) ISRCTN82853500. Pediatr Crit Care Med 19:939-948

259. Piastra M, De Luca D, Pietrini D et al (2009) Noninvasive pressuresupport ventilation in immunocompromised children with ARDS: a feasibility study. Intensive Care Med 35:1420-1427

260. Essouri S, Carroll C, Pediatric Acute Lung Injury Consensus Conference Group (2015) Noninvasive support and ventilation for pediatric acute respiratory distress syndrome: proceedings from the Pediatric Acute Lung Injury Consensus Conference. Pediatr Crit Care Med 16:S102-S110

261. Kneyber MCJ, de Luca D, Calderini E, Section Respiratory Failure of the European Society for Paediatric and Neonatal Intensive Care et al (2017) Recommendations for mechanical ventilation of critically ill children from the Paediatric Mechanical Ventilation Consensus Conference (PEMVECC). Intensive Care Med 43:1764-1780

262. Brower RG, Matthay MA, Morris A, Acute Respiratory Distress Syndrome Network et al (2000) Ventilation with lower tidal volumes as compared with traditional tidal volumes for acute lung injury and the acute respiratory distress syndrome. N Engl J Med 342:1301-1308

263. Curley MA, Hibberd PL, Fineman LD et al (2005) Effect of prone positioning on clinical outcomes in children with acute lung injury: a randomized controlled trial. JAMA 294:229-237

264. Newth CJL, Sward KA, Khemani RG, Eunice Kennedy Shriver National Institute for Child Health and Human Development Collaborative Pediatric Critical Care Research Network (CPCCRN) et al (2017) Variability in usual care mechanical ventilation for pediatric acute respiratory distress syndrome: time for a decision support protocol? Pediatr Crit Care Med 18:e521-e529

265. Erickson S, Schibler A, Numa A, Paediatric Study Group; Australian and New Zealand Intensive Care Society et al (2007) Acute lung injury in pediatric intensive care in Australia and New Zealand: a prospective, multicenter, observational study. Pediatr Crit Care Med 8:317-323

266. Flori HR, Glidden DV, Rutherford GW et al (2005) Pediatric acute lung injury: prospective evaluation of risk factors associated with mortality. Am J Respir Crit Care Med 171:995-1001

267. Parvathaneni K, Belani S, Leung D et al (2017) Evaluating the performance of the pediatric acute lung injury consensus conference definition of acute respiratory distress syndrome. Pediatr Crit Care Med 18:17-25

268. Miller MP, Sagy M (2008) Pressure characteristics of mechanical ventilation and incidence of pneumothorax before and after the implementation of protective lung strategies in the management of pediatric patients with severe ARDS. Chest 134:969-973

269. Santschi M, Jouvet P, Leclerc F, PALIVE Investigators; Pediatric Acute Lung Injury and Sepsis Investigators Network (PALISI); European Society of Pediatric and Neonatal Intensive Care (ESPNIC) et al (2010) Acute lung injury in children: Therapeutic practice and feasibility of international clinical trials. Pediatr Crit Care Med 11:681-689

270. Khemani RG, Markovitz BP, Curley MAQ (2009) Characteristics of children intubated and mechanically ventilated in 16 PICUs. Chest 136:765-771

271. López-Fernández Y, Azagra AM, de la Oliva P, Pediatric Acute Lung Injury Epidemiology and Natural History (PED-ALIEN) Network et al (2012) Pediatric Acute Lung Injury Epidemiology and Natural History study: Incidence and outcome of the acute respiratory distress syndrome in children. Crit Care Med 40:3238-3245

272. Ingaramo OA, Ngo T, Khemani RG et al (2014) Impact of positive endexpiratory pressure on cardiac index measured by ultrasound cardiac output monitor. Pediatr Crit Care Med 15:15-20

273. Guo F, Hao L, Zhen Q et al (2016) Multicenter study on the prognosis associated with respiratory support for children with acute hypoxic respiratory failure. Exp Ther Med 12:3227-3232

274. Yehya N, Thomas NJ (2017) Disassociating lung mechanics and oxygenation in pediatric acute respiratory distress syndrome. Crit Care Med 45:1232-1239

275. Yehya N, Thomas NJ, Khemani RG (2018) Risk stratification using oxygenation in the first 24 hours of pediatric acute respiratory distress syndrome. Crit Care Med 46:619-624

276. Khemani RG, Parvathaneni K, Yehya N et al (2018) Positive end-expiratory pressure lower than the ARDS network protocol is associated with higher pediatric acute respiratory distress syndrome mortality. Am J Respir Crit Care Med 198:77-89

277. Halbertsma FJ, Vaneker M, Pickkers P et al (2010) A single recruitment maneuver in ventilated critically ill children can translocate pulmonary cytokines into the circulation. J Crit Care 25:10-15

278. Wolf GK, Gómez-Laberge C, Kheir JN et al (2012) Reversal of dependent lung collapse predicts response to lung recruitment in children with early acute lung injury. Pediatr Crit Care Med 13:509-515

279. Goligher EC, Hodgson CL, Adhikari NKJ et al (2017) Lung recruitment maneuvers for adult patients with acute respiratory distress syndrome. A systematic review and meta-analysis. Ann Am Thorac Soc 14:S304-S311

280. Boriosi JP, Sapru A, Hanson JH et al (2011) Efficacy and safety of lung recruitment in pediatric patients with acute lung injury. Pediatr Crit Care Med 12:431-436

281. Duff JP, Rosychuk RJ, Joffe AR (2007) The safety and efficacy of sustained inflations as a lung recruitment maneuver in pediatric intensive care unit patients. Intensive Care Med 33:1778-1786

282. Cavalcanti AB, Suzumura EA, Laranjeira LN, Writing Group for the Alveolar Recruitment for Acute Respiratory Distress Syndrome Trial (ART) Investigators et al (2017) Effect of lung recruitment and titrated positive end-expiratory pressure (PEEP) vs low PEEP on mortality in patients with acute respiratory distress syndrome: a randomized clinical trial. JAMA 318:1335-1345 
283. Kang H, Yang $H$, Tong $Z$ (2019) Recruitment manoeuvres for adults with acute respiratory distress syndrome receiving mechanical ventilation: a systematic review and meta-analysis. J Crit Care 50:1-10

284. Santini A, Protti A, Langer T et al (2015) Prone position ameliorates lung elastance and increases functional residual capacity independently from lung recruitment. Intensive Care Med Exp 3:55

285. Sud S, Friedrich JO, Adhikari NK et al (2014) Effect of prone positioning during mechanical ventilation on mortality among patients with acute respiratory distress syndrome: a systematic review and meta-analysis. CMAJ 186:E381-E390

286. Sud S, Friedrich JO, Taccone P et al (2010) Prone ventilation reduces mortality in patients with acute respiratory failure and severe hypoxemia: systematic review and meta-analysis. Intensive Care Med 36:585-599

287. Kornecki A, Frndova $H$, Coates AL et al (2001) 4A randomized trial of prolonged prone positioning in children with acute respiratory failure. Chest 119:211-218

288. Macrae DJ, Field D, Mercier JC et al (2004) Inhaled nitric oxide therapy in neonates and children: reaching a European consensus. Intensive Care Med 30:372-380

289. Day RW, Lynch JM, White KS et al (1996) Acute response to inhaled nitric oxide in newborns with respiratory failure and pulmonary hypertension. Pediatrics 98:698-705

290. Dobyns EL, Cornfield DN, Anas NG et al (1999) Multicenter randomized controlled trial of the effects of inhaled nitric oxide therapy on gas exchange in children with acute hypoxemic respiratory failure. J Pediatr 134:406-412

291. Bronicki RA, Fortenberry J, Schreiber M et al (2015) Multicenter randomized controlled trial of inhaled nitric oxide for pediatric acute respiratory distress syndrome. J Pediatr 166(365-369):e1

292. Karam O, Gebistorf F, Wetterslev J et al (2017) The effect of inhaled nitric oxide in acute respiratory distress syndrome in children and adults: a cochrane systematic review with trial sequential analysis. Anaesthesia 72:106-117

293. Fioretto JR, de Moraes MA, Bonatto RC et al (2004) Acute and sustained effects of early administration of inhaled nitric oxide to children with acute respiratory distress syndrome. Pediatr Crit Care Med 5:469-474

294. Tadphale SD, Rettiganti M, Gossett JM et al (2016) Is administration of nitric oxide during extracorporeal membrane oxygenation associated with improved patient survival? Pediatr Crit Care Med 17:1080-1087

295. Dowell JC, Thomas NJ, Yehya N (2017) Association of response to inhaled nitric oxide and duration of mechanical ventilation in pediatric acute respiratory distress syndrome. Pediatr Crit Care Med 18:1019-1026

296. Bhalla AK, Yehya N, Mack WJ et al (2018) The association between inhaled nitric oxide treatment and ICU mortality and 28-day ventilator-free days in pediatric acute respiratory distress syndrome. Crit Care Med 46:1803-1810

297. Gebistorf F, Karam O, Wetterslev J et al (2016) Inhaled nitric oxide for acute respiratory distress syndrome (ARDS) in children and adults. Cochrane Database Syst Rev 66:CD002787

298. Ferguson ND, Cook DJ, Guyatt GH, OSCILLATE Trial Investigators; Canadian Critical Care Trials Group et al (2013) High-frequency oscillation in early acute respiratory distress syndrome. N Engl I Med 368:795-805

299. Young D, Lamb SE, Shah S et al (2013) OSCAR Study Group: highfrequency oscillation for acute respiratory distress syndrome. N Engl J Med 368:806-813

300. Guo YX, Wang ZN, Li YT et al (2016) High-frequency oscillatory ventilation is an effective treatment for severe pediatric acute respiratory distress syndrome with refractory hypoxemia. Ther Clin Risk Manag 12:1563-1571

301. Bateman ST, Borasino S, Asaro LA, RESTORE Study Investigators et al (2016) Early high-frequency oscillatory ventilation in pediatric acute respiratory failure. A propensity score analysis. Am J Respir Crit Care Med 193:495-503

302. Arnold JH, Hanson JH, Toro-Figuero LO et al (1994) Prospective, randomized comparison of high-frequency oscillatory ventilation and conventional mechanical ventilation in pediatric respiratory failure. Crit Care Med 22:1530-1539
303. Samransamruajkit R, Prapphal N, Deelodegenavong J et al (2005) Plasma soluble intercellular adhesion molecule-1 (sICAM-1) in pediatric ARDS during high frequency oscillatory ventilation: a predictor of mortality. Asian Pac J Allergy Immunol 23:181-188

304. Samransamruajkit R, Rassameehirun C, Pongsanon K et al (2016) A comparison of clinical efficacy between high frequency oscillatory ventilation and conventional ventilation with lung volume recruitment in pediatric acute respiratory distress syndrome: a randomized controlled trial. Indian J Crit Care Med 20:72-77

305. Gainnier M, Roch A, Forel JM et al (2004) Effect of neuromuscular blocking agents on gas exchange in patients presenting with acute respiratory distress syndrome. Crit Care Med 32:113-119

306. Forel JM, Roch A, Marin V et al (2006) Neuromuscular blocking agents decrease inflammatory response in patients presenting with acute respiratory distress syndrome. Crit Care Med 34:2749-2757

307. Papazian L, Forel JM, Gacouin A, ACURASYS Study Investigators et al (2010) Neuromuscular blockers in early acute respiratory distress syndrome. N Engl J Med 363:1107-1116

308. Wilsterman MEF, de Jager P, Blokpoel R et al (2016) Short-term effects of neuromuscular blockade on global and regional lung mechanics, oxygenation and ventilation in pediatric acute hypoxemic respiratory failure. Ann Intensive Care 6:103

309. Da Silva PS, Neto HM, de Aguiar VE et al (2010) Impact of sustained neuromuscular blockade on outcome of mechanically ventilated children. Pediatr Int 52:438-443

310. Wehling M (1997) Specific, nongenomic actions of steroid hormones. Annu Rev Physiol 59:365-393

311. Seri I, Evans J (2001) Controversies in the diagnosis and management of hypotension in the newborn infant. Curr Opin Pediatr 13:116-123

312. Munck A, Mendel DB, Smith LI et al (1990) Glucocorticoid receptors and actions. Am Rev Respir Dis 141:S2-10

313. Sasidharan P (1998) Role of corticosteroids in neonatal blood pressure homeostasis. Clin Perinatol 25(723-740):xi

314. Yung M, Wilkins B, Norton L, Paediatric Study Group; Australian and New Zealand Intensive Care Society et al (2008) Glucose control, organ failure, and mortality in pediatric intensive care. Pediatr Crit Care Med 9:147-152

315. Kwon S, Hermayer KL, Hermayer K (2013) Glucocorticoid-induced hyperglycemia. Am J Med Sci 345:274-277

316. Milan G, Romanello V, Pescatore F et al (2015) Regulation of autophagy and the ubiquitin-proteasome system by the FoxO transcriptional network during muscle atrophy. Nat Commun 6:6670

317. Steiner SJ, Noe JD, Denne SC (2011) Corticosteroids increase protein breakdown and loss in newly diagnosed pediatric crohn disease. Pediatr Res 70:484-488

318. Costello JM, Graham DA, Morrow DF et al (2009) Risk factors for central line-associated bloodstream infection in a pediatric cardiac intensive care unit. Pediatr Crit Care Med 10:453-459

319. Waljee AK, Rogers MA, Lin P et al (2017) Short term use of oral corticosteroids and related harms among adults in the United States: population based cohort study. BMJ 357:j1415

320. El-Nawawy A, Khater D, Omar H et al (2017) Evaluation of early corticosteroid therapy in management of pediatric septic shock in pediatric intensive care patients: a randomized clinical study. Pediatr Infect Dis J 36:155-159

321. Rygård SL, Butler E, Granholm A et al (2018) Low-dose corticosteroids for adult patients with septic shock: a systematic review with meta-analysis and trial sequential analysis. Intensive Care Med 44:1003-1016

322. Annane D, Sébille V, Charpentier C et al (2002) Effect of treatment with low doses of hydrocortisone and fludrocortisone on mortality in patients with septic shock. JAMA 288:862-871

323. Sprung CL, Annane D, Keh D, CORTICUS Study Group et al (2008) Hydrocortisone therapy for patients with septic shock. N Engl J Med 358:111-124

324. Annane D, Renault A, Brun-Buisson C, CRICS-TRIGGERSEP Network et al (2018) Hydrocortisone plus fludrocortisone for adults with septic shock. N Engl J Med 378:809-818

325. Venkatesh B, Finfer S, Cohen J, ADRENAL Trial Investigators and the Australian-New Zealand Intensive Care Society Clinical Trials Group 
et al (2018) Adjunctive glucocorticoid therapy in patients with septic shock. N Engl J Med 378:797-808

326. Yehya N, Vogiatzi MG, Thomas NJ et al (2016) Cortisol correlates with severity of illness and poorly reflects adrenal function in pediatric acute respiratory distress syndrome. J Pediatr 177(212-218):e1

327. Rochwerg B, Oczkowski SJ, Siemieniuk RAC et al (2018) Corticosteroids in sepsis: an updated systematic review and meta-analysis. Crit Care Med 46:1411-1420

328. Lamontagne F, Rochwerg B, Lytvyn L et al (2018) Corticosteroid therapy for sepsis: a clinical practice guideline. BMJ 362:k3284

329. Slusher T, Gbadero D, Howard C et al (1996) Randomized, placebocontrolled, double blinded trial of dexamethasone in African children with sepsis. Pediatr Infect Dis J 15:579-583

330. Valoor HT, Singhi S, Jayashree M (2009) Low-dose hydrocortisone in pediatric septic shock: an exploratory study in a third world setting. Pediatr Crit Care Med 10:121-125

331. Menon K, McNally D, O'Hearn K, Canadian Critical Care Trials Group et al (2017) A randomized controlled trial of corticosteroids in pediatric septic shock: A pilot feasibility study. Pediatr Crit Care Med 18:505-512

332. Markovitz BP, Goodman DM, Watson RS et al (2005) A retrospective cohort study of prognostic factors associated with outcome in pediatric severe sepsis: what is the role of steroids? Pediatr Crit Care Med 6:270-274

333. Zimmerman JJ, Williams MD (2011) Adjunctive corticosteroid therapy in pediatric severe sepsis: observations from the RESOLVE study. Pediatr Crit Care Med 12:2-8

334. Atkinson SJ, Cvijanovich NZ, Thomas NJ et al (2014) Corticosteroids and pediatric septic shock outcomes: a risk stratified analysis. PLoS One 9:e112702

335. Wong HR, Cvijanovich NZ, Anas N et al (2015) Developing a clinically feasible personalized medicine approach to pediatric septic shock. Am J Respir Crit Care Med 191:309-315

336. Menon K, MCNally JD, Choong K, Canadian Critical Care Trials Group STRIPES Investigators et al (2015) A cohort study of pediatric shock: Frequency of corticosteriod use and association with clinical outcomes. Shock 44:402-409

337. Bornstein SR, Allolio B, Arlt W et al (2016) Diagnosis and treatment of primary adrenal insufficiency: an endocrine society clinical practice guideline. J Clin Endocrinol Metab 101:364-389

338. Broersen LH, Pereira AM, Jørgensen JO et al (2015) Adrenal insufficiency in corticosteroids use: systematic review and meta-analysis. J Clin Endocrinol Metab 100:2171-2180

339. Agus MS, Steil GM, Wypij D, SPECS Study Investigators et al (2012) Tight glycemic control versus standard care after pediatric cardiac surgery. $\mathrm{N}$ Engl J Med 367:1208-1219

340. Agus MSD, Wypij D, Nadkarni VM (2017) Tight glycemic control in critically ill children. N Engl J Med 376:e48

341. Macrae D, Grieve R, Allen E, CHiP Investigators et al (2014) A randomized trial of hyperglycemic control in pediatric intensive care. N Engl Med 370:107-118

342. Vlasselaers D, Milants I, Desmet L et al (2009) Intensive insulin therapy for patients in paediatric intensive care: a prospective, randomised controlled study. Lancet 373:547-556

343. Jeschke MG, Kulp GA, Kraft R et al (2010) Intensive insulin therapy in severely burned pediatric patients: a prospective randomized trial. Am J Respir Crit Care Med 182:351-359

344. Chen L, Li T, Fang F et al (2018) Tight glycemic control in critically ill pediatric patients: a systematic review and meta-analysis. Crit Care 22:57

345. Zhao Y, Wu Y, Xiang B (2018) Tight glycemic control in critically ill pediatric patients: a meta-analysis and systematic review of randomized controlled trials. Pediatr Res 84:22-27

346. McKinlay CJD, Alsweiler JM, Anstice NS, Children With Hypoglycemia and Their Later Development (CHYLD) Study Team et al (2017) Association of neonatal glycemia with neurodevelopmental outcomes at 4.5 years. JAMA Pediatr 171:972-983

347. Mesotten D, Gielen M, Sterken C et al (2012) Neurocognitive development of children 4 years after critical illness and treatment with tight glucose control: a randomized controlled trial. JAMA 308:1641-1650
348. Sadhwani A, Asaro LA, Goldberg C et al (2016) Impact of tight glycemic control on neurodevelopmental outcomes at 1 year of age for children with congenital heart disease: a randomized controlled trial. J Pediatr 174(193-198):e2

349. Faustino EVS, Hirshberg EL, Asaro LA, Heart and lung failure-pediatric insulin titration (HALF-PINT) study investigators et al (2019) Shortterm adverse outcomes associated with hypoglycemia in critically ill children. Crit Care Med 47:706-714

350. Forsythe RM, Wessel CB, Billiar TR et al (2008) Parenteral calcium for intensive care unit patients. Cochrane Database Syst Rev CD006163

351. Dotson B, Larabell P, Patel JU et al (2016) Calcium administration is associated with adverse outcomes in critically ill patients receiving parenteral nutrition: results from a natural experiment created by a calcium gluconate shortage. Pharmacotherapy 36:1185-1190

352. Dias CR, Leite HP, Nogueira PC et al (2013) lonized hypocalcemia is an early event and is associated with organ dysfunction in children admitted to the intensive care unit. J Crit Care 28:810-815

353. Hebbar K, Rigby MR, Felner El et al (2009) Neuroendocrine dysfunction in pediatric critical illness. Pediatr Crit Care Med 10:35-40

354. Shih JL, Agus MS (2009) Thyroid function in the critically ill newborn and child. Curr Opin Pediatr 21:536-540

355. Marwali EM, Boom CE, Sakidjan I et al (2013) Oral triiodothyronine normalizes triiodothyronine levels after surgery for pediatric congenital heart disease*. Pediatr Crit Care Med 14:701-708

356. Marwali EM, Boom CE, Budiwardhana N et al (2017) Oral triiodothyronine for infants and children undergoing cardiopulmonary bypass. Ann Thorac Surg 104:688-695

357. Smith LM, Leake RD, Berman N et al (2000) Postnatal thyroxine supplementation in infants less than 32 weeks' gestation: effects on pulmonary morbidity. J Perinatol 20:427-431

358. Lee BH, Inui D, Suh GY, Fever and Antipyretic in Critically ill patients Evaluation (FACE) Study Group et al (2012) Association of body temperature and antipyretic treatments with mortality of critically ill patients with and without sepsis: Multi-centered prospective observational study. Crit Care 16:R33

359. Walter EJ, Hanna-Jumma S, Carraretto M et al (2016) The pathophysiological basis and consequences of fever. Crit Care 20:200

360. Sullivan JE, Farrar HC, Section on clinical pharmacology and therapeutics, committee on drugs (2011) Fever and antipyretic use in children. Pediatrics 127:580-587

361. Drewry AM, Ablordeppey EA, Murray ET et al (2017) Antipyretic therapy in critically ill septic patients: a systematic review and meta-analysis. Crit Care Med 45:806-813

362. Prakash V, Parameswaran N, Biswal N (2016) Early versus late enteral feeding in critically ill children: a randomized controlled trial. Intensive Care Med 42:481-482

363. Rice TW, Mogan S, Hays MA et al (2011) Randomized trial of initial trophic versus full-energy enteral nutrition in mechanically ventilated patients with acute respiratory failure. Crit Care Med 39:967-974

364. Meyer R, Harrison S, Sargent S et al (2009) The impact of enteral feeding protocols on nutritional support in critically ill children. J Hum Nutr Diet 22:428-436

365. Petrillo-Albarano T, Pettignano R, Asfaw M et al (2006) Use of a feeding protocol to improve nutritional support through early, aggressive, enteral nutrition in the pediatric intensive care unit. Pediatr Crit Care Med 7:340-344

366. Yoshimura S, Miyazu M, Yoshizawa S et al (2015) Efficacy of an enteral feeding protocol for providing nutritional support after paediatric cardiac surgery. Anaesth Intensive Care 43:587-593

367. Hamilton S, McAleer DM, Ariagno K et al (2014) A stepwise enteral nutrition algorithm for critically ill children helps achieve nutrient delivery goals*. Pediatr Crit Care Med 15:583-589

368. Panchal AK, Manzi J, Connolly S et al (2016) Safety of enteral feedings in critically ill children receiving vasoactive agents. JPEN J Parenter Enteral Nutr 40:236-241

369. King W, Petrillo T, Pettignano R (2004) Enteral nutrition and cardiovascular medications in the pediatric intensive care unit. JPEN J Parenter Enteral Nutr 28:334-338

370. López-Herce J, Santiago MJ, Sánchez C et al (2008) Risk factors for gastrointestinal complications in critically ill children with transpyloric enteral nutrition. Eur J Clin Nutr 62:395-400 
371. Arabi YM, Aldawood AS, Haddad SH, PermiT Trial Group et al (2015) Permissive underfeeding or standard enteral feeding in critically ill adults. N Engl J Med 372:2398-2408

372. Fivez T, Kerklaan D, Mesotten D et al (2016) Early versus late parenteral nutrition in critically ill children. N Engl J Med 374:1111-1122

373. van Puffelen E, Vanhorebeek I, Joosten KFM et al (2018) Early versus late parenteral nutrition in critically ill, term neonates: a preplanned secondary subgroup analysis of the PEPaNIC multicentre, randomised controlled trial. Lancet Child Adolesc Health 2:505-515

374. van Puffelen E, Hulst JM, Vanhorebeek I et al (2018) Outcomes of delaying parenteral nutrition for 1 week vs initiation within 24 hours among undernourished children in pediatric intensive care: a subanalysis of the PEPaNIC randomized clinical trial. JAMA Netw Open 1:e182668

375. Verstraete S, Verbruggen SC, Hordijk JA et al (2019) Long-term developmental effects of withholding parenteral nutrition for 1 week in the paediatric intensive care unit: a 2-year follow-up of the PEPaNIC international, randomised, controlled trial. Lancet Respir Med 7:141-153

376. Doig GS, Simpson F, Sweetman EA, Early PN Investigators of the ANZICS Clinical Trials Group et al (2013) Early parenteral nutrition in critically ill patients with short-term relative contraindications to early enteral nutrition: a randomized controlled trial. JAMA 309:2130-2138

377. Casaer MP, Mesotten D, Hermans G et al (2011) Early versus late parenteral nutrition in critically ill adults. N Engl J Med 365:506-517

378. Singer P, Anbar R, Cohen J et al (2011) The tight calorie control study (TICACOS): a prospective, randomized, controlled pilot study of nutritional support in critically ill patients. Intensive Care Med 37:601-609

379. Briassoulis G, Filippou O, Hatzi E et al (2005) Early enteral administration of immunonutrition in critically ill children: results of a blinded randomized controlled clinical trial. Nutrition 21:799-807

380. Carcillo JA, Dean JM, Holubkov R, Eunice Kennedy Shriver National Institute of Child Health and Human Development (NICHD) Collaborative Pediatric Critical Care Research Network (CPCCRN) et al (2012) The randomized comparative pediatric critical illness stress-induced immune suppression (CRISIS) prevention trial. Pediatr Crit Care Med 13:165-173

381. Al-Biltagi MA, Abo-Elezz AA, Abd-Elhafez MA et al (2017) Beneficial effects of omega-3 supplement to the enteral feeding in children with mild to moderate sepsis. J Intensive Care Med 32:212-217

382. Tume LN, Bickerdike A, Latten L et al (2017) Routine gastric residual volume measurement and energy target achievement in the PICU: a comparison study. Eur J Pediatr 176:1637-1644

383. Meert KL, Daphtary KM, Metheny NA (2004) Gastric vs small-bowel feeding in critically ill children receiving mechanical ventilation: a randomized controlled trial. Chest 126:872-878

384. Kamat P, Favaloro-Sabatier J, Rogers K et al (2008) Use of methylene blue spectrophotometry to detect subclinical aspiration in enterally fed intubated pediatric patients. Pediatr Crit Care Med 9:299-303

385. Sönmez Düzkaya D, Yildiz S (2016) Effect of two different feeding methods on preventing ventilator associated pneumonia in the paediatric intensive care unit (PICU): a randomised controlled study. Aust Crit Care 29:139-145

386. Lewis K, Alqahtani Z, Mcintyre L et al (2016) The efficacy and safety of prokinetic agents in critically ill patients receiving enteral nutrition: a systematic review and meta-analysis of randomized trials. Crit Care 20:259

387. Yap YG, Camm AJ (2003) Drug induced QT prolongation and torsades de pointes. Heart 89:1363-1372

388. Freeman BD, Dixon DJ, Coopersmith CM et al (2008) Pharmacoepidemiology of QT-interval prolonging drug administration in critically ill patients. Pharmacoepidemiol Drug Saf 17:971-981

389. Ray WA, Murray KT, Meredith S et al (2004) Oral erythromycin and the risk of sudden death from cardiac causes. N Engl J Med 351:1089-1096

390. Stadtman TC (1996) Selenocysteine. Annu Rev Biochem 65:83-100

391. Iglesias SB, Leite HP, Paes AT et al (2014) Low plasma selenium concentrations in critically ill children: the interaction effect between inflammation and selenium deficiency. Crit Care 18:R101

392. Loui A, Raab A, Braetter P et al (2008) Selenium status in term and preterm infants during the first months of life. Eur J Clin Nutr 62:349-355
393. Sammalkorpi K, Valtonen V, Alfthan G et al (1988) Serum selenium in acute infections. Infection 16:222-224

394. Asci A, Surmeli-Onay O, Erkekoglu P et al (2015) Oxidant and antioxidant status in neonatal proven and clinical sepsis according to selenium status. Pediatr Int 57:1131-1137

395. Mertens K, Lowes DA, Webster NR et al (2015) Low zinc and selenium concentrations in sepsis are associated with oxidative damage and inflammation. Br J Anaesth 114:990-999

396. Darlow BA, Austin NC (2003) Selenium supplementation to prevent short-term morbidity in preterm neonates. Cochrane Database Syst Rev CD003312

397. Alhazzani W, Almasoud A, Jaeschke R et al (2013) Small bowel feeding and risk of pneumonia in adult critically ill patients: a systematic review and meta-analysis of randomized trials. Crit Care 17:R127

398. Barbosa E, Moreira EA, Goes JE et al (1999) Pilot study with a glutaminesupplemented enteral formula in critically ill infants. Rev Hosp Clin Fac Med Sao Paulo 54:21-24

399. Briassoulis G, Filippou O, Kanariou M et al (2005) Comparative effects of early randomized immune or non-immune-enhancing enteral nutrition on cytokine production in children with septic shock. Intensive Care Med 31:851-858

400. Heyland D, Muscedere J, Wischmeyer PE, Canadian Critical Care Trials Group et al (2013) A randomized trial of glutamine and antioxidants in critically ill patients. N Engl J Med 368:1489-1497

401. Jordan I, Balaguer M, Esteban ME et al (2016) Glutamine effects on heat shock protein 70 and interleukines 6 and 10: randomized trial of glutamine supplementation versus standard parenteral nutrition in critically ill children. Clin Nutr 35:34-40

402. Novak F, Heyland DK, Avenell A et al (2002) Glutamine supplementation in serious illness: a systematic review of the evidence. Crit Care Med 30:2022-2029

403. van Zanten AR, Sztark F, Kaisers UX et al (2014) High-protein enteral nutrition enriched with immune-modulating nutrients vs standard high-protein enteral nutrition and nosocomial infections in the ICU: a randomized clinical trial. JAMA 312:514-524

404. Wernerman J, Kirketeig T, Andersson B, Scandinavian Critical Care Trials Group et al (2011) Scandinavian glutamine trial: a pragmatic multicentre randomised clinical trial of intensive care unit patients. Acta Anaesthesiol Scand 55:812-818

405. Beale RJ, Sherry T, Lei K et al (2008) Early enteral supplementation with key pharmaconutrients improves sequential organ failure assessment score in critically ill patients with sepsis: outcome of a randomized, controlled, double-blind trial. Crit Care Med 36:131-144

406. Fuentes-Orozco C, Anaya-Prado R, González-Ojeda A et al (2004) L-alanyl-L-glutamine-supplemented parenteral nutrition improves infectious morbidity in secondary peritonitis. Clin Nutr 23:13-21

407. Koksal GM, Erbabacan E, Tunali Y et al (2014) The effects of intravenous, enteral and combined administration of glutamine on malnutrition in sepsis: a randomized clinical trial. Asia Pac J Clin Nutr 23:34-40

408. Bollhalder L, Pfeil AM, Tomonaga $Y$ et al (2013) A systematic literature review and meta-analysis of randomized clinical trials of parenteral glutamine supplementation. Clin Nutr 32:213-223

409. Tao KM, Li XQ, Yang LQ et al (2014) Glutamine supplementation for critically ill adults. Cochrane Database Syst Rev 9:CD010050

410. Tubman TR, Thompson SW, McGuire W (2008) Glutamine supplementation to prevent morbidity and mortality in preterm infants. Cochrane Database Syst Rev 1:CD001457.

411. Carcillo JA, Dean JM, Holubkov R, Eunice Kennedy Shriver National Institute of Child Health and Human Development (NICHD) Collaborative Pediatric Critical Care Research Network (CPCCRN) et al (2017) Interaction between 2 nutraceutical treatments and host immune status in the pediatric critical illness stress-induced immune suppression comparative effectiveness trial. JPEN J Parenter Enteral Nutr 41:1325-1335

412. Barbul A, Uliyargoli A (2007) Use of exogenous arginine in multiple organ dysfunction syndrome and sepsis. Crit Care Med 35:S564-S567

413. Bertolini G, lapichino G, Radrizzani D et al (2003) Early enteral immunonutrition in patients with severe sepsis: results of an interim analysis of a randomized multicentre clinical trial. Intensive Care Med 29:834-840 
414. Suchner U, Kuhn KS, Fürst P (2000) The scientific basis of immunonutrition. Proc Nutr Soc 59:553-563

415. Argaman Z, Young VR, Noviski N et al (2003) Arginine and nitric oxide metabolism in critically ill septic pediatric patients. Crit Care Med 31:591-597

416. Bower RH, Cerra FB, Bershadsky B et al (1995) Early enteral administration of a formula (impact) supplemented with arginine, nucleotides, and fish oil in intensive care unit patients: results of a multicenter, prospective, randomized, clinical trial. Crit Care Med 23:436-449

417. Caparrós T, Lopez J, Grau T (2001) Early enteral nutrition in critically ill patients with a high-protein diet enriched with arginine, fiber, and antioxidants compared with a standard high-protein diet. The effect on nosocomial infections and outcome. JPEN J Parenter Enteral Nutr 25:299-308

418. Galbán C, Montejo JC, Mesejo A et al (2000) An immune-enhancing enteral diet reduces mortality rate and episodes of bacteremia in septic intensive care unit patients. Crit Care Med 28:643-648

419. Santora R, Kozar RA (2010) Molecular mechanisms of pharmaconutrients. J Surg Res 161:288-294

420. Kieft H, Roos AN, van Drunen JD et al (2005) Clinical outcome of immunonutrition in a heterogeneous intensive care population. Intensive Care Med 31:524-532

421. Taylor BE, McClave SA, Martindale RG et al, Society of Critical Care Medicine, American Society of Parenteral and Enteral Nutrition (2016) Guidelines for the provision and assessment of nutrition support therapy in the adult critically ill patient: Society of Critical Care Medicine (SCCM) and American Society for Parenteral and Enteral Nutrition (A.S.P.E.N.). Crit Care Med 44:390-438

422. Langlois P, Lamontagne F (2019) Vitamin C for the critically ill: Is the evidence strong enough? Nutrition 60:185-190

423. Marik PE (2018) Vitamin C for the treatment of sepsis: the scientific rationale. Pharmacol Ther 189:63-70

424. Teng J, Pourmand A, Mazer-Amirshahi M (2018) Vitamin C: the next step in sepsis management? J Crit Care 43:230-234

425. Carr AC, Rosengrave PC, Bayer $S$ et al (2017) Hypovitaminosis $C$ and vitamin $C$ deficiency in critically ill patients despite recommended enteral and parenteral intakes. Crit Care 21:300

426. Fowler AA 3rd, Syed AA, Knowlson S, Medical Respiratory Intensive Care Unit Nursing et al (2014) Phase I safety trial of intravenous ascorbic acid in patients with severe sepsis. J Transl Med 12:32

427. Zabet MH, Mohammadi M, Ramezani M et al (2016) Effect of high-dose ascorbic acid on vasopressor's requirement in septic shock. J Res Pharm Pract 5:94-100

428. Moskowitz A, Andersen LW, Huang DT et al (2018) Ascorbic acid, corticosteroids, and thiamine in sepsis: a review of the biologic rationale and the present state of clinical evaluation. Crit Care 22:283

429. Marik PE, Khangoora V, Rivera R et al (2017) Hydrocortisone, vitamin $C$, and thiamine for the treatment of severe sepsis and septic shock: a retrospective before-after study. Chest 151:1229-1238

430. Donnino MW, Andersen LW, Chase M, Center for Resuscitation Science Research Group et al (2016) Randomized, double-blind, placebocontrolled trial of thiamine as a metabolic resuscitator in septic shock: a pilot study. Crit Care Med 44:360-367

431. Lima LF, Leite HP, Taddei JA (2011) Low blood thiamine concentrations in children upon admission to the intensive care unit: risk factors and prognostic significance. Am J Clin Nutr 93:57-61

432. Costa NA, Gut AL, de Souza Dorna M et al (2014) Serum thiamine concentration and oxidative stress as predictors of mortality in patients with septic shock. J Crit Care 29:249-252

433. McNally JD, Menon K, Chakraborty P, Canadian Critical Care Trials Group et al (2012) The association of vitamin D status with pediatric critical illness. Pediatrics 130:429-436

434. Madden K, Feldman HA, Smith EM et al (2012) Vitamin D deficiency in critically ill children. Pediatrics 130:421-428

435. Sankar J, Lotha W, Ismail J et al (2016) Vitamin D deficiency and length of pediatric intensive care unit stay: a prospective observational study. Ann Intensive Care 6:3

436. Ponnarmeni S, Kumar Angurana S, Singhi S et al (2016) Vitamin D deficiency in critically ill children with sepsis. Paediatr Int Child Health 36:15-21
437. Onwuneme C, Carroll A, Doherty D et al (2015) Inadequate vitamin D levels are associated with culture positive sepsis and poor outcomes in paediatric intensive care. Acta Paediatr 104:e433-e438

438. Madden K, Feldman HA, Chun RF et al (2015) Critically ill children have low vitamin D-binding protein, influencing bioavailability of vitamin $D$. Ann Am Thorac Soc 12:1654-1661

439. McNally JD, lliriani K, Pojsupap S et al (2015) Rapid normalization of vitamin D levels: a meta-analysis. Pediatrics 135:e152-e166

440. McNally D, Amrein K, O'Hearn K, Canadian Critical Care Trials Group et al (2017) Study protocol for a phase II dose evaluation randomized controlled trial of cholecalciferol in critically ill children with vitamin D deficiency (VITdAL-PICU study). Pilot Feasibility Stud 3:70

441. McNally JD (2016) Vitamin D deficiency in pediatric critical illness: time to move on from observational studies? Rev Chil Pediatr 87:439-441

442. Marcinowska-Suchowierska E, Kupisz-Urbańska M, Łukaszkiewicz J et al (2018) Vitamin D toxicity-a clinical perspective. Front Endocrinol (Lausanne) 9:550

443. Narsaria P, Sankar J, Lodha R (2016) Fatal outcome of accidental vitamin D overdose. Indian J Pediatr 83:1040

444. Misra M, Pacaud D, Petryk A, Drug and Therapeutics Committee of the Lawson Wilkins Pediatric Endocrine Society et al (2008) Vitamin D deficiency in children and its management: review of current knowledge and recommendations. Pediatrics 122:398-417

445. Karam O, Tucci M, Ducruet T, Canadian Critical Care Trials Group; PALISI Network et al (2011) Red blood cell transfusion thresholds in pediatric patients with sepsis. Pediatr Crit Care Med 12:512-518

446. Lacroix J, Hébert PC, Hutchison JS, TRIPICU Investigators; Canadian Critical Care Trials Group; Pediatric Acute Lung Injury and Sepsis Investigators Network et al (2007) Transfusion strategies for patients in pediatric intensive care units. N Engl J Med 356:1609-1619

447. Hébert PC, Wells G, Blajchman MA et al (1999) A multicenter, randomized, controlled clinical trial of transfusion requirements in critical care. Transfusion requirements in Critical Care Investigators, Canadian Critical Care Trials Group. N Engl J Med 340:409-417

448. Muszynski JA, Guzzetta NA, Hall MW, Pediatric Critical Care Transfusion and Anemia Expertise Initiative (TAXI); Pediatric Critical Care Blood Research Network (Blood Net), and the Pediatric Acute Lung Injury and Sepsis Investigators (PALISI) Network et al (2018) Recommendations on $\mathrm{RBC}$ transfusions for critically ill children with nonhemorrhagic shock from the pediatric critical care transfusion and anemia expertise initiative. Pediatr Crit Care Med 19:S121-S126

449. Sankar J, Sankar MJ, Suresh CP et al (2014) Early goal-directed therapy in pediatric septic shock: comparison of outcomes "with" and "without" intermittent superior venacaval oxygen saturation monitoring: a prospective cohort study. Pediatr Crit Care Med 15:e157-e167

450. Holst LB, Haase N, Wetterslev J, TRISS Trial Group; Scandinavian Critical Care Trials Group et al (2014) Lower versus higher hemoglobin threshold for transfusion in septic shock. N Engl J Med 371:1381-1391

451. Du Pont-Thibodeau G, Tucci M, Robitaille N et al (2016) Platelet transfusions in pediatric intensive care. Pediatr Crit Care Med 17:e420-e429

452. Curley A, Stanworth SJ, Willoughby K, PlaNeT2 MATISSE Collaborators et al (2019) Randomized trial of platelet-transfusion thresholds in neonates. N Engl J Med 380:242-251

453. Pieters BJ, Conley L, Weiford J et al (2015) Prophylactic versus reactive transfusion of thawed plasma in patients undergoing surgical repair of craniosynostosis: a randomized clinical trial. Paediatr Anaesth 25:279-287

454. Yang L, Stanworth S, Hopewell S et al (2012) Is fresh-frozen plasma clinically effective? An update of a systematic review of randomized controlled trials. Transfusion 52:1673-1686

455. Church GD, Matthay MA, Liu K et al (2009) Blood product transfusions and clinical outcomes in pediatric patients with acute lung injury. Pediatr Crit Care Med 10:297-302

456. Karam O, Lacroix J, Robitaille $\mathrm{N}$ et al (2013) Association between plasma transfusions and clinical outcome in critically ill children: a prospective observational study. Vox Sang 104:342-349

457. Abdel-Wahab OI, Healy B, Dzik WH (2006) Effect of fresh-frozen plasma transfusion on prothrombin time and bleeding in patients with mild coagulation abnormalities. Transfusion 46:1279-1285 
458. Rimmer E, Houston BL, Kumar A et al (2014) The efficacy and safety of plasma exchange in patients with sepsis and septic shock: a systematic review and meta-analysis. Crit Care 18:699

459. Schwartz J, Padmanabhan A, Aqui N et al (2016) Guidelines on the use of therapeutic apheresis in clinical practice-evidence-based approach from the Writing Committee of the American Society for Apheresis: the seventh special issue. J Clin Apher 31:149-162

460. Nguyen TC, Han YY, Kiss JE et al (2008) Intensive plasma exchange increases a disintegrin and metalloprotease with thrombospondin motifs-13 activity and reverses organ dysfunction in children with thrombocytopenia-associated multiple organ failure. Crit Care Med 36:2878-2887

461. Carcillo JA, Berg RA, Wessel D, Eunice Kennedy Shriver National Institute of Child Health and Human Development Collaborative Pediatric Critical Care Research Network et al (2019) A multicenter network assessment of three inflammation phenotypes in pediatric sepsis-induced multiple organ failure. Pediatr Crit Care Med 20:1137-1146

462. Nguyen TC, Liu A, Liu L et al (2007) Acquired ADAMTS-13 deficiency in pediatric patients with severe sepsis. Haematologica 92:121-124

463. Bernardo A, Ball C, Nolasco L et al (2004) Effects of inflammatory cytokines on the release and cleavage of the endothelial cellderived ultralarge von Willebrand factor multimers under flow. Blood 104:100-106

464. Ono T, Mimuro J, Madoiwa S et al (2006) Severe secondary deficiency of von Willebrand factor-cleaving protease (ADAMTS13) in patients with sepsis-induced disseminated intravascular coagulation: its correlation with development of renal failure. Blood 107:528-534

465. Crawley JT, Lam JK, Rance JB et al (2005) Proteolytic inactivation of ADAMTS13 by thrombin and plasmin. Blood 105:1085-1093

466. Studt JD, Kremer Hovinga JA, Antoine G et al (2005) Fatal congenital thrombotic thrombocytopenic purpura with apparent ADAMTS13 inhibitor: in vitro inhibition of ADAMTS13 activity by hemoglobin. Blood 105:542-544

467. Nolasco LH, Turner NA, Bernardo A et al (2005) Hemolytic uremic syndrome-associated Shiga toxins promote endothelial-cell secretion and impair ADAMTS13 cleavage of unusually large von Willebrand factor multimers. Blood 106:4199-4209

468. Tsai HM, Lian EC (1998) Antibodies to von Willebrand factor-cleaving protease in acute thrombotic thrombocytopenic purpura. N Engl J Med 339:1585-1594

469. Fortenberry JD, Nguyen T, Grunwell JR, Thrombocytopenia-Associated Multiple Organ Failure (TAMOF) Network Study Group et al (2019) Therapeutic plasma exchange in children with thrombocytopeniaassociated multiple organ failure: The Thrombocytopenia-Associated Multiple Organ Failure Network prospective experience. Crit Care Med 47:e173-e181

470. Sevketoglu E, Yildizdas D, Horoz OO et al (2014) Use of therapeutic plasma exchange in children with thrombocytopenia-associated multiple organ failure in the Turkish thrombocytopenia-associated multiple organ failure network. Pediatr Crit Care Med 15:e354-e359

471. Rock GA, Shumak KH, Buskard NA et al (1991) Comparison of plasma exchange with plasma infusion in the treatment of thrombotic thrombocytopenic purpura. Canadian Apheresis Study Group. N Engl J Med 325:393-397

472. Guo XH, Sun YF, Han SZ et al (2017) Continuous blood purification in children with severe sepsis. J Biol Regul Homeost Agents 31:389-394

473. Sutherland SM, Zappitelli M, Alexander SR et al (2010) Fluid overload and mortality in children receiving continuous renal replacement therapy: the prospective pediatric continuous renal replacement therapy registry. Am J Kidney Dis 55:316-325

474. Gulla KM, Sachdev A, Gupta D et al (2015) Continuous renal replacement therapy in children with severe sepsis and multiorgan dysfunction-a pilot study on timing of initiation. Indian J Crit Care Med 19:613-617

475. Borthwick EM, Hill CJ, Rabindranath KS et al (2017) High-volume haemofiltration for sepsis in adults. Cochrane Database Syst Rev 1:CD008075

476. Miao H, Wang F, Xiong X et al (2018) Clinical benefits of high-volume hemofiltration in critically ill pediatric patients with severe sepsis: a Retrospective Cohort Study. Blood Purif 45:18-27
477. Nasr VG, Raman L, Barbaro RP et al (2019) Highlights from the extracorporeal life support organization registry: 2006-2017. ASAIO J 65:537-544

478. Maclaren G, Butt W, Best D et al (2007) Extracorporeal membrane oxygenation for refractory septic shock in children: one institution's experience. Pediatr Crit Care Med 8:447-451

479. Ruth A, McCracken CE, Fortenberry JD et al (2015) Extracorporeal therapies in pediatric severe sepsis: findings from the pediatric health-care information system. Crit Care 19:397

480. Skinner SC, locono JA, Ballard HO et al (2012) Improved survival in venovenous vs venoarterial extracorporeal membrane oxygenation for pediatric noncardiac sepsis patients: a study of the Extracorporeal Life Support Organization registry. J Pediatr Surg 47:63-67

481. Barbaro RP, Xu Y, Borasino S, RESTORE Study Investigators et al (2018) Does extracorporeal membrane oxygenation improve survival in pediatric acute respiratory failure? Am J Respir Crit Care Med 197:1177-1186

482. Curley MA, Wypij D, Watson RS, RESTORE Study Investigators and the Pediatric Acute Lung Injury and Sepsis Investigators Network et al (2015) Protocolized sedation vs usual care in pediatric patients mechanically ventilated for acute respiratory failure: a randomized clinical trial. JAMA 313:379-389

483. Bailly DK, Reeder RW, Winder M, Eunice Kennedy Shriver National Institute of Child Health and Human Development (NICHD) Collaborative Pediatric Critical Care Research Network (CPCCRN) et al (2019) Development of the pediatric extracorporeal membrane oxygenation prediction model for risk-adjusting mortality. Pediatr Crit Care Med 20:426-434

484. Jen HC, Shew SB (2010) Hospital readmissions and survival after nonneonatal pediatric ECMO. Pediatrics 125:1217-1223

485. Barbaro RP, Odetola FO, Kidwell KM et al (2015) Association of hospitallevel volume of extracorporeal membrane oxygenation cases and mortality. Analysis of the extracorporeal life support organization registry. Am J Respir Crit Care Med 191:894-901

486. MacLaren G, Butt W, Best D et al (2011) Central extracorporeal membrane oxygenation for refractory pediatric septic shock. Pediatr Crit Care Med 12:133-136

487. Bréchot N, Luyt CE, Schmidt M et al (2013) Venoarterial extracorporeal membrane oxygenation support for refractory cardiovascular dysfunction during severe bacterial septic shock. Crit Care Med 41:1616-1626

488. Oberender F, Ganeshalingham A, Fortenberry JD et al (2018) Venoarterial extracorporeal membrane oxygenation versus conventional therapy in severe pediatric septic shock. Pediatr Crit Care Med 19:965-972

489. Ohlsson A, Lacy JB (2013) Intravenous immunoglobulin for suspected or proven infection in neonates. Cochrane Database Syst Rev CD001239

490. El-Nawawy A, El-Kinany H, Hamdy El-Sayed M et al (2005) Intravenous polyclonal immunoglobulin administration to sepsis syndrome patients: a prospective study in a pediatric intensive care unit. J Trop Pediatr 51:271-278

491. Brocklehurst P, Farrell B, King A et al (2011) INIS Collaborative Group: treatment of neonatal sepsis with intravenous immune globulin. N Engl Med 365:1201-1211

492. Alejandria MM, Lansang MA, Dans LF et al (2013) Intravenous immunoglobulin for treating sepsis, severe sepsis and septic shock. Cochrane Database Syst Rev CD001090

493. Berlot G, Vassallo MC, Busetto N et al (2018) Effects of the timing of administration of IgM- and IgA-enriched intravenous polyclonal immunoglobulins on the outcome of septic shock patients. Ann Intensive Care 8:122

494. Parks T, Wilson C, Curtis N et al (2018) Polyspecific intravenous immunoglobulin in clindamycin-treated patients with streptococcal toxic shock syndrome: a systematic review and meta-analysis. Clin Infect Dis 67:1434-1436

495. Kadri SS, Swihart BJ, Bonne SL et al (2017) Impact of intravenous immunoglobulin on survival in necrotizing fasciitis with vasopressordependent shock: a propensity score-matched analysis from 130 US hospitals. Clin Infect Dis 64:877-885

496. Madsen MB, Lange T, Hjortrup PB et al (2016) Immunoglobulin for necrotising soft tissue infections (INSTINCT): protocol for a randomised trial. Dan Med J 63:A5250 
497. Reveiz L, Guerrero-Lozano R, Camacho A et al (2010) Stress ulcer, gastritis, and gastrointestinal bleeding prophylaxis in critically ill pediatric patients: a systematic review. Pediatr Crit Care Med 11:124-132

498. Jimenez J, Drees M, Loveridge-Lenza B et al (2015) Exposure to gastric acid-suppression therapy is associated with health care- and community-associated Clostridium difficile infection in children. J Pediatr Gastroenterol Nutr 61:208-211

499. Cook D, Heyland D, Griffith L et al (1999) Risk factors for clinically important upper gastrointestinal bleeding in patients requiring mechanical ventilation. Canadian Critical Care Trials Group. Crit Care Med 27:2812-2817

500. Duerksen DR (2003) Stress-related mucosal disease in critically ill patients. Best Pract Res Clin Gastroenterol 17:327-344

501. Marik PE, Vasu T, Hirani A et al (2010) Stress ulcer prophylaxis in the new millennium: a systematic review and meta-analysis. Crit Care Med $38: 2222-2228$
502. Massicotte P, Julian JA, Gent M, PROTEKT Study Group et al (2003) An open-label randomized controlled trial of low molecular weight heparin for the prevention of central venous line-related thrombotic complications in children: The PROTEKT trial. Thromb Res 109:101-108

503. Brandao LR, Shah N, Shah PS (2014) Low molecular weight heparin for prevention of central venous catheterization-related thrombosis in children. Cochrane Database Syst Rev CD005982

504. van Ommen CH, Heijboer H, Büller HR et al (2001) Venous thromboembolism in childhood: a prospective two-year registry in The Netherlands. J Pediatr 139:676-681

505. Raffini L, Huang YS, Witmer C et al (2009) Dramatic increase in venous thromboembolism in children's hospitals in the United States from 2001 to 2007. Pediatrics 124:1001-1008

506. Takemoto CM, Sohi S, Desai K et al (2014) Hospital-associated venous thromboembolism in children: incidence and clinical characteristics. $J$ Pediatr 164:332-338 